\title{
UNIVERSITÄT
}

LEIPZIG

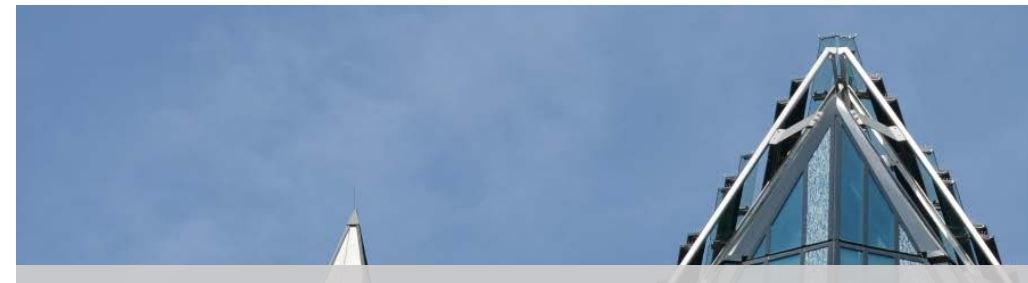

\section{Der Breitbandausbau in Sachsen} Hemmnisse, Handlungsoptionen und die Rolle des Tiefbaus

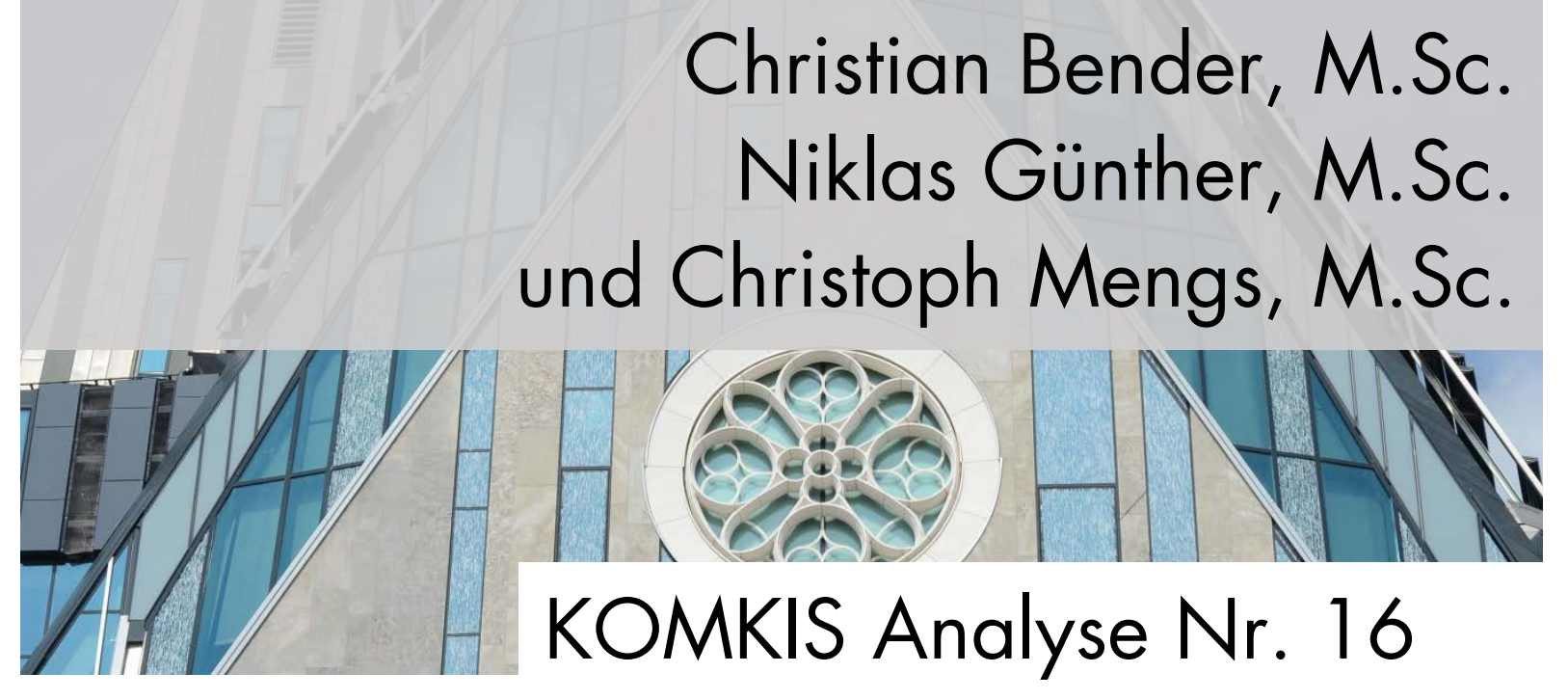




\section{KOMKIS Analyse | Der Breitbandausbau in Sachsen}

Bender, Christian, M. Sc., wissenschaftlicher Mitarbeiter am Kompetenzzentrum für kommunale Infrastruktur Sachsen.

Günther, Niklas, M. Sc., wissenschaftlicher Mitarbeiter am Kompetenzzentrum für kommunale Infrastruktur Sachsen.

Mengs, Christoph, M. Sc., wissenschaftlicher Mitarbeiter am Kompetenzzentrum für kommunale Infrastruktur Sachsen.

unter Mitarbeit von Moritz Emlein und Sina Rühland

Bibliographische Information der Deutschen Nationalbibliothek: Die Deutsche Nationalbibliothek verzeichnet diese Publikation in der Deutschen Nationalbibliographie; detaillierte bibliographische Daten sind im Internet über http:// dnb.d-nb. de abrufbar.

\section{ISSN 2511-7432}

Erscheinungsjahr 2020

Wenn Sie auf diese KOMKIS-Publikation direkt über einen Digital Object Identifier (DOI) verweisen wollen, nutzen Sie bitte folgenden Link:

https: / / doi. org/ 10.36730/ 2020.4. komkisa. 16

\section{Zitierempfehlung:}

Bender, Christian/Günther, Niklas/ Mengs, Christoph (2020): Der Breitbandausbau in Sachsen - Hemmnisse, Handlungsoptionen und die Rolle des Tiefbaus, KOMKIS Analyse, Nr. 16, Leipzig.

Ansprechperson: Christian Bender

T +49 341 9733-628 | F +49341 9733-589 | bender@wifa.uni-leipzig.de

\section{SACHSEN}

Diese Publikation wird mitfinanziert durch Steuermittel auf der Grundlage des von den Abgeordneten des Sächsischen Landtags beschlossenen Haushalts.

(c) 2020 Kompetenzzentrum für kommunale Infrastruktur Sachsen,

Städtisches Kaufhaus, Universitätsstraße 16, 04109 Leipzig. Alle Rechte vorbehalten.

Foto: Paul Trainer | Universität Leipzig 


\section{Inhaltsverzeichnis}

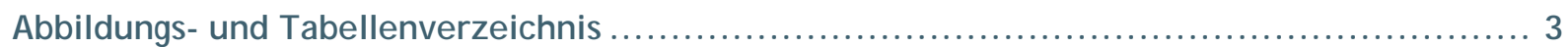

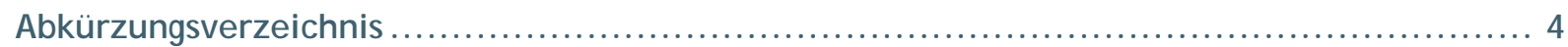

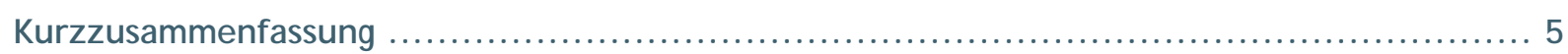

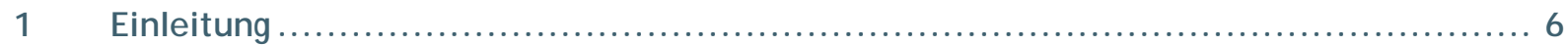

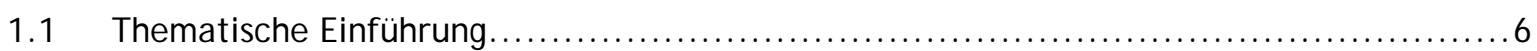

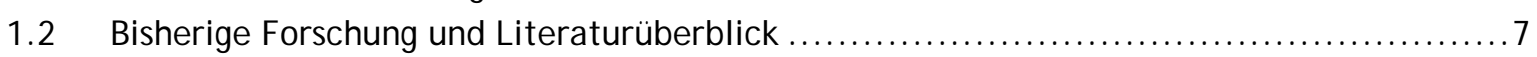

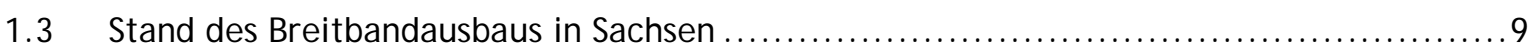

2 Die Rolle der Tiefbaumaßnahmen für den Breitbandausbau.............................. 15

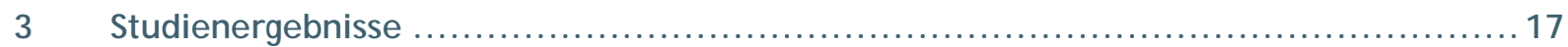

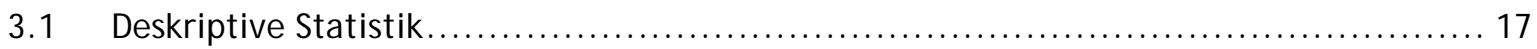

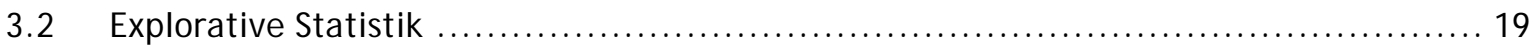

3.2.1 Teil A: Allgemeine Angaben ................................................... 20

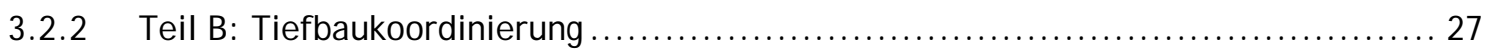

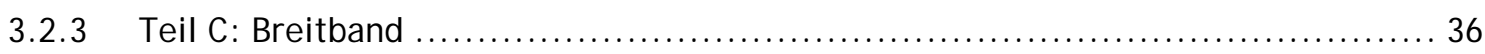

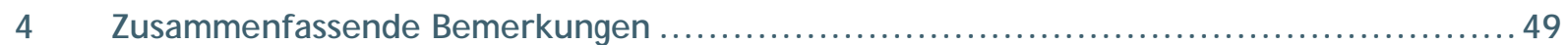

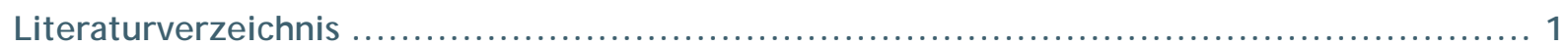

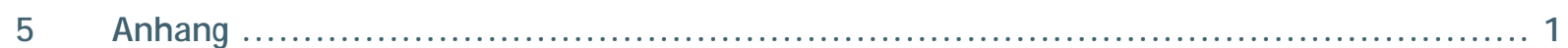

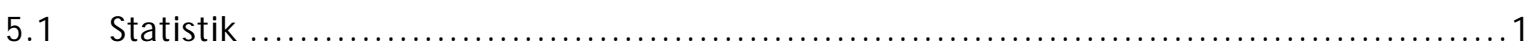

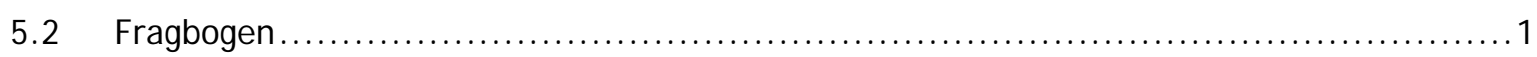




\section{Abbildungs- und Tabellenverzeichnis}

Abbildung 1: Breitbandverfügbarkeit der Haushalte und Gewerbestandorte in Flächenländern ( $\geq 50 \mathrm{Mbit} / \mathrm{s}$, alle Technologien) nach Ländern im J ahr 2019

Abbildung 2: Breitbandverfügbarkeit der Haushalte in Flächenländern nach leitungsgebundenen

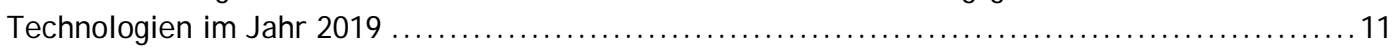

Abbildung 3: Breitbandverfügbarkeit der Gewerbestandorte in Flächenländern nach leitungsgebundenen

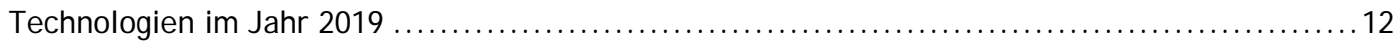

Abbildung 4: Breitbandverfügbarkeit der Haushalte und Gewerbestandorte in Sachsen (alle Technologien)

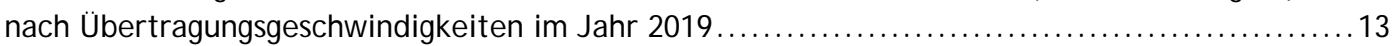

Abbildung 5: Karte der Breitbandverfügbarkeit für Privathaushalte in Sachsen ( $\geq 50 \mathrm{Mbit} / \mathrm{s}$, alle

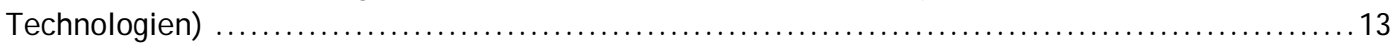

Abbildung 6: Karte der Breitbandverfügbarkeit für Gewerbestandorte in Sachsen ( $\geq 50 \mathrm{Mbit} / \mathrm{s}$, alle

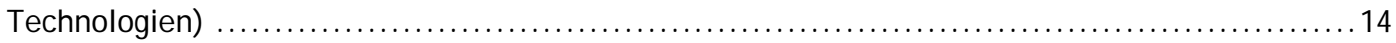

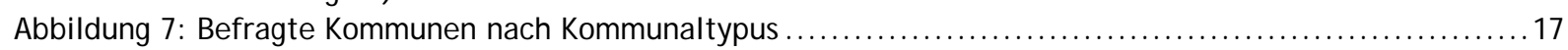

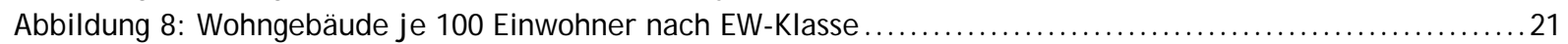

Abbildung 9: Siedlungs- und Verkehrsfläche je 1000 Einwohner nach EW-Klasse ............................ 22

Abbildung 10: Hochgerechneter Straßenbestand nach Typus in km nach EW-Klassen.........................23

Abbildung 11: Hochgerechnete VZÄ (Bau-/ Hauptamt) je 1000 Einwohner nach EW-Klassen ....................24

Abbildung 12: Hochgerechneter Anteil der leitungsgebundenen Breitbandverfügbarkeit von $50 \mathrm{Mbit} / \mathrm{s}$ der privaten Haushalte und des Gewerbes................................................ 26

Abbildung 13: Führen Sie verschiedene Baumaßnahmen im Tiefbau innerhalb Ihrer Kommune oder im Rahmen einer interkommunalen Kooperation gebündelt durch? ......................... 28

Abbildung 14: Stimmen Sie aus Sicht Ihrer Kommune zu, dass die folgenden Hindernisse im Rahmen der Koordinierung von Tiefbaumaßnahmen bestehen? ................................... 30

Abbildung 15: Wie schätzen Sie die Instrumente zur Förderung der Koordinierung im kommunalen Tiefbau

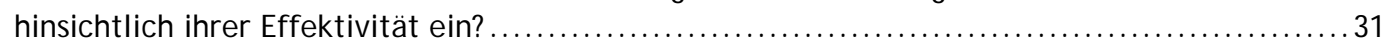

Abbildung 16: Gibt es eine Dokumentation über vorhandene Leitungen oder anderer Infrastrukturen in

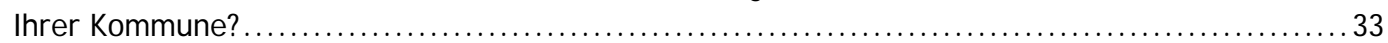

Abbildung 17: Wie ist der Zugang zu den Informationen für Dritte zurzeit in Ihrer Kommune gestaltet? .........34

Abbildung 18: Wie schätzen Sie die Bedeutung der Speicherung folgender Informationen hinsichtlich ihrer Wichtigkeit für eine erfolgreiche Koordinierung von kommunalen Tiefbaumaßnahmen ein? ...... 35

Abbildung 19: Wie bewerten Sie die nachfolgenden Faktoren für den lokalen Breitbandausbau bezogen auf

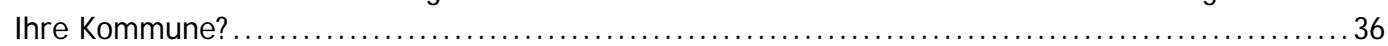

Abbildung 20: Für welche Bereiche schätzen Sie die Bedeutung des Breitbandausbaus aus Sicht Ihrer Kommune als besonders hoch ein?

Abbildung 21: Haben Sie als Kommune oder eines Ihrer kommunalen Unternehmen bereits ein oder mehrere Projekte zum Breitbandausbau durchgeführt oder begleitet? ..................... 38

Abbildung 22: Für wie wichtig schätzen Sie aus Sicht Ihrer Kommune die folgenden Hemmnisse des öffentlich geförderten Breitbandausbaus ein? .......................................... 39

Abbildung 23: Stimmen Sie aus Sicht Ihrer Kommune zu, das die folgenden Hindernisse im Rahmen der Fördermittelprogrammatik, beispielsweise der Digitale Offensive Sachsen, bestehen? .........41

Abbildung 24: Wie schätzen Sie Instrumente zur Förderung des Breitbandausbaus hinsichtlich ihrer Effektivität ein?

Abbildung 25: Welche Verwaltungsebene sollte diese Maßnahme aus Sicht Ihrer Kommune hauptsächlich durchführen?

Abbildung 26: Für wie hilfreiche schätzen Sie die Umsetzungshilfen des Gesetzes zur Erleichterung des Ausbaus digitaler Hochgeschwindigkeitsnetze (DigiNetzG) ein? ....................... 46

Abbildung 27: Für wie hilfreich schätzen Sie die Zusammenarbeit mit den folgenden Partnern für Unterstützung, Planung oder Umsetzung eines Breitbandausbauprojektes ein? ............... 48

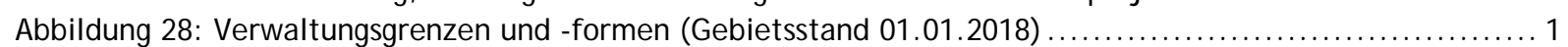

Tabelle 1: Deskriptive Statistik der metrischen Merkmalsträger.... 19 


\section{Abkürzungsverzeichnis}

(V)DSL Very High Speed Digital Subscriber Line

Abs. . Absatz

BB ...... Brandenburg

BW. Baden-Württemberg

BY Bayern bzw beziehungsweise

ca. circa

CATV Kabelfernsehen DigiNetzG.

e. V Gesetzes zur Erleichterung des Ausbaus digitaler Hochgeschwindigkeitsnetze

et al. eingetragener Verein

EU ...... Europäische Union

EW Einwohner FTTB/ H . Fibre to the building/ home

Gbit/S Gbits pro Sekunde

GIS. Geoinformationssystem $\mathrm{GmbH}$ Gesellschaft mit beschränkter Haftung HE . Hessen $\mathrm{km}$. Kilometer Mbit/s Megabits pro Sekunde Mio. Millionen MV . Mecklenburg-Vorpommern $\mathrm{NI}$ Niedersachsen

NW . Nordrhein-Westfalen ÖPNV Öffentlicher Personennahverkehr

OZG Onlinezugangsgesetz RP Rheinland-Pfalz Sächsisches Gesetz über kommunale Zusammenarbeit $\mathrm{SH}$ Schleswig-Holstein SL Saarland

SN Sachsen sogenannt 


\section{Kurzzusammenfassung}

Die vorliegende KOMKIS Analyse bewertet auf Grundlage der Ergebnisse einer durchgeführten Befragung der kommunalen Bau- bzw. Hauptämter im Freistaat Sachsen die aktuellen Einschätzungen und Meinungen der kommunalen Ebene im Kontext des Tiefbaus sowie des geförderten Breitbandausbaus. Hierbei werden die beiden Themenfelder nicht isoliert betrachtet, sondern Zusammenhänge, wie ähnliche Herausforderungen, herausgearbeitet. Die vorliegende Analyse baut somit auf den bisherigen Erkenntnissen des KOMKIS auf und ergänzt das bereits bestehende Bild zum kommunalen Breitbandausbau im Freistaat Sachsen.

Die Bundesregierung setzte sich zum Ziel, eine gigabitfähige Breitbandinfrastruktur flächendeckend bereitzustellen. Neben dem privatwirtschaftlichen Ausbau wird die kommunale Bautätigkeit durch Fördermittel des Bundes und der Länder unterstützt. Die Strategie des Freistaats Sachsen unterstützt dieses Vorgehen. Zwar sind in Sachsen einige Erfolge hinsichtlich des Breitbandausbaus zu verzeichnen, dennoch hinkt der Anteil der Verfügbarkeit von Anschlüssen mit hohen Internetgeschwindigkeiten den Zielen deutlich hinterher. Die Divergenz zwischen dem städtischen und ländlichen Raum fällt in Sachsen im Vergleich mit anderen Ländern stark aus. Gegenwärtig wird die Bedeutung von Hochgeschwindigkeitsinternet als Standortfaktor für Gewerbe und Privathaushashalte besonders deutlich.

Als zentrale Gründe werden häufig die Überlastung des Tiefbausektors und die Nichtinanspruchnahme von Fördermitteln durch die Kommunen angeführt. Die vorliegende Analyse geht einen Schritt weiter und stellt die wesentlichen Paralallen zwischen den Themenfeldern Breitbandausbau und Tiefbau dar. Beispielhaft sind hier Kapazitätsengpässe, Größenvorteile und Koordinierungsproblematiken zu nennen. Die Befragung der Tief- bzw. Hauptämter der sächsischen Städte und Gemeinden bildet die Grundlage für die statistische Auswertung.

Es wird deutlich, dass der Bündelung von kommunalen Tiefbaumaßnamen in Sachsen kein Vorrang eingeräumt wird. Kleinere und zersiedelte Kommunen nutzen jedoch bereits häufiger diese Möglichkeit. Unterstützend wirkt unter anderem eine Dokumentation der vorhandenen Leitungen, die in den Kommunen zumeist als öffentlich-zugängliche GIS-basierte Karten realisiert werden.

Lokale Standortfaktoren, die das Angebot an Breitband beeinflussen, bestimmen die lokale Versorgung an Hochgeschwindigkeitsinternet maßgeblich. Diese sind relativ inflexibel und nur mithilfe politischer Maßnahmen zu verändern. Durch die Digitalisierung der Koordinierung könnten Synergien geschaffen werden, die den weiteren Breitbandausbau unterstützen würden. 


\section{Einleitung}

\subsection{Thematische Einführung}

Zugriffsmöglichkeiten auf schnelle Internetverbindungen stellen gegenwärtig einen bedeutsamen Standortfaktor für gewerbliches Wachstum und die Gestaltung des Privatlebens dar. Die Breitbandverfügbarkeit konzentriert sich laut der übergreifenden Zielsetzung der Bundesregierung auf die flächendeckende Verfügbarkeit von gigabitfähiger Infrastruktur. Vorrangig soll diese in einem wettbewerblichen Umfeld über einen privatwirtschaftlichen Ausbau geschehen. Ergänzt werden diese Investitionen durch einen kommunalen Ausbau mithilfe von Fördermitteln des Bundes und der Länder. Zudem sollen sogenannte "weiße Flecken“, demnach Gebiete mit Anschlussgeschwindigkeiten von unter $30 \mathrm{Mbit} / \mathrm{s}$, und sozioökonomische Treiber, wie Gewerbegebiete, gezielt gefördert werden. Eine erweiterte Förderkulisse zur Schließung von sog. „grauen Flecken“ (Anschlüsse zwischen $30 \mathrm{Mbit} / \mathrm{s}$ und $1 \mathrm{Gbit} / \mathrm{s}$ ) besteht seitens der EU-Kommission. ${ }^{1}$

In den sächsischen Städten und Gemeinden kommt die Digitalisierung, gemessen am Breitbandausbau, nur schleppend voran. Die Breitbandverfügbarkeit divergiert laut aktueller Statistik des BMVI dabei sehr zwischen den urbanen und ländlichen Gebieten. Bezüglich des Zugangs zu Hochgeschwindigkeitsinternet, mit Übertragungsraten von mehr als $50 \mathrm{Mbit} / \mathrm{s}$, weist insbesondere der ländliche Raum im Freistaat Sachsen mit einer Abdeckung von lediglich 55,1\% gegenüber dem bundesweiten Durchschnitt von 69,4\% Abdeckung einen deutlichen Nachholbedarf auf. ${ }^{2}$

Zudem zeichnet sich der Markt für Tiefbauleistungen derzeit durch eine stetig hohe Nachfrage aus. Für private Tiefbauunternehmen ist bspw. der Breitbandausbau, speziell der Ausbau von Glasfaserkabelnetzen, wirtschaftlich wenig rentabel, da u. a. durch regionale Spezifika ein besonderes Maß an (kostenintensiver) Flexibilität erforderlich ist, was im Vergleich zu anderen Tiefbauvorhaben deutlich geringere Gewinnmargen zulässt. Zugleich erfordert der Ausbau eine weitreichende Fachexpertise. Bezogen auf die entsprechenden Fachkräfte zeigt sich dort neben einem allgemeinen Arbeitskräftemangel der auch in anderen Branchen bestehende Mangel. ${ }^{3}$

Trotz des bestehenden Nachholbedarfs werden die Fördermittel zum Breitbandausbau, die vom Bund und dem Freistaat Sachsen zur Verfügung gestellt werden, zuletzt nur in geringen

Siehe Bundesregierung (2020).

Vgl. BMWI (2019).

Vgl. Rottmann/ Neuhäuser (2019), S. 9. 
Maße von den Kommunen in Anspruch genommen. ${ }^{4}$ Zudem wird die Möglichkeit, die Breitbandnetze selbst zu betreiben, von den Kommunen nur spärlich genutzt. ${ }^{5}$ Vor dem Hintergrund der wenig beanspruchten Fördermittelprogramme setzt die vorliegende Kommunalbefragung an, um Aufschluss über die Hintergründe zu geben. Es erfolgte eine Teilerhebung der sächsischen Kommunen mit einer möglichst zufälligen Auswahl über einen standardisierten Fragebogen. Die Adressaten des Fragebogens waren alle Verantwortlichen für den Breitbandausbau bzw. Tiefbaumaßnahmen der sächsischen Kommunen, die die Möglichkeit hatten, anonymisiert an der schriftlichen Befragung teilzunehmen. Mithilfe dieser Herangehensweise wird über ein möglichst breites Spektrum ein Gesamtbild der Problemlage mit vergleichbaren Daten generiert.

Die übergeordneten Forschungsfragen lauten:

- Lassen sich Hemmnisse für den Breitbandausbau in Sachsen identifizieren?

- Erfolgt eine Koordinierung kommunaler Tiefbaumaßnahmen? Wie wird die Dokumentation organisiert?

- Wie ließe sich der Breitbandausbau durch (Einzel-)Maßnahmen und Institutionen weiter unterstützen?

\subsection{Bisherige Forschung und Literaturüberblick}

Im Rahmen der 2018er KOMKIS Analyse zum Breitbandausbau in Sachsen wurde herausgestellt, dass besonders die Raumstruktur und die Art der Flächennutzung einen Einfluss auf den marktgetrieben erfolgten Netzausbau des Hochgeschwindigkeitsinternets in Sachsen haben. Die Studie zeigt den digital divide, folglich das Gefälle der Verfügbarkeit höherer Breitbandgeschwindigkeiten zwischen städtischen Zentren und peripheren, ländlichen Regionen. Zudem werden die beiden Aspekte der Liberalisierung des Telekommunikationsmarktes und der Breitbandversorgung als Universaldienst historisch wie juristisch eingeordnet. Mithilfe von Daten, die die Raumstruktur sowie demographischen und sozioökonomischen Strukturen erfassen, wurden statistische Analysen auf kommunaler Ebene für die J ahre 2011 bis 2017 durchgeführt. Als Ergebnis konnten mehrere Faktoren identifiziert werden, die das Angebot des Breitbands im Untersuchungszeitraum signifikant beeinflusst haben: Die Einflussfaktoren sind vor allem auf der Angebotsseite relevant. Beispielsweise sind hier die Siedlungsstruktur (erfasst durch den Anteil an Ein- und Zweifamilienhäusern sowie den Zersiedlungsindex), die Investitionskosten und die Dichte an potentiellen Nutzern (gemessen in Einwohnern und Erwerbstätigen und der Siedlungs- und Verkehrsfläche) der Ortschaft zu nennen. Die Faktoren

Siehe Bundestag (2019).

5 Siehe Breitbandkompetenzzentrum Sachsen (2019). 
auf der Nachfrageseite, wie qualifizierte Beschäftigte, sind weniger bzw. gar nicht signifikant. Auch im Zeitablauf ändert sich diese Beobachtung nicht, sodass bei jedem neuen Ausbauziel die alten Hindernisse bestehen bleiben. ${ }^{6}$

Das KOMKIS erhebt zudem in regelmäßigen Abständen die Investitions- und Instandhaltungsbedarfe für die kommunale Ebene im Freistaat. Dabei zeigt sich, dass sich der kommunale Investitionsbedarf für den Breitbandausbau sachsenweit innerhalb von drei J ahren deutlich gesteigert hat: 2016 betrug der Investitionsbedarf noch ca. 517 Mio. Euro, wohingegen er in 2018 auf ca. 776 Mio. Euro gestiegen ist. Im J ahr 2020 sank dieser nur unwesentlich auf 710 Mio. Euro. Insgesamt ist ein Zehntel des kommunalen Investitionsbedarfs in Sachsen auf die Breitbandinfrastruktur zurückzuführen. ${ }^{7}$

Das INSTITUT FÜR INNOVATION UND TECHNIK führte mit dem DEUTSCHEN STÄDTE- UND GEMEINDEBUND im Jahr 2019 eine bundesweite Umfrage mit dem Titel „Zukunftsradar Digitale Kommune“ durch. Diese Befragung zielte auf die Digitalisierung in Kommunalverwaltungen. Zwar liegt ein abweichender Themenschwerpunkt vor, jedoch beleuchtete die Studie ähnliche Inhalte und nutzte die gleiche Methode, wie die vorliegende Studie. Zusammengefasst wird deutlich, dass der Breitbandausbau für viele der befragten Kommunen nach wie vor eine bedeutende Rolle spielt. Der größte Handlungsbedarf wird bei der Digitalisierung der Verwaltung und beim Breitbandausbau wahrgenommen. Dabei wird der Nutzen der Digitalisierung, aber gleichzeitig auch der Finanzierungsbedarf von einem Großteil der befragten Städte und Gemeinden als hoch eingeschätzt. Als Erfolgsfaktor wird die interkommunale Vernetzung gesehen, gefolgt von zusätzlichen Finanzmitteln und externen Beratungsleistungen. ${ }^{8}$

In einer Verbundstudie des KompetenZZENTRUMS ÖFFENTLICHE WIRTSCHAFT, INFRASTRUKTUR UND DASEINSVORSORGE E. V. an der Universität Leipzig zusammen mit der PSPC GMBH wurden mögliche Beschleunigungsfaktoren und Hemmnisse der Förderung des Breitbandausbaus im ländlichen Raum untersucht. Zusätzlich zu einer fragebogengestützten Kommunalbefragung wurden Experteninterviews mit den (Fremd- und Eigen-)Kapitalgebern durchgeführt. Als mögliche Hemmnisse des Breitbandausbaus wurden die eine komplexe und langwierige Förderkulisse, der fehlende strategische Überbau, ein unsicheres Regulierungsumfeld (insbesondere der regulatorische Rahmen von Mitnutzungen und Mitverlegungen) sowie fehlende Finanzierungsmöglichkeiten identifiziert. ${ }^{9}$

6 Vgl. Mengs/ Günther (2018).

7 Die Angaben beziehen sich auf das genannte und die folgenden drei J ahre ab dem genannten Zeitpunkt. Vgl. Sydow/ Hesse (2018), S. 14 f.; Sydow/ Hesse (2020), S. 16 f.

$8 \mathrm{Vgl}$. Handschuh et al. (2019).

9 Vgl. Rottmann/ Hilbig (2019). 
Der Bundesverband Breitbandkommunikation (BREKO) befragte für die Studie "MarktanaIyse19“ die 183 deutschlandweit tätigen Netzbetreiber, die Mitglied in diesem Verband sind. Laut der präsentierten Ergebnissen stellt weniger die Verfügbarkeit von öffentlichen Geldern als vielmehr die komplexe Fördermittelvergabe ein Hemmnis dar. Ferner sind der vielerorts zu beobachtende Doppelausbau und knappe Tiefbaukapazitäten problematisch. Die ansteigende Breitbandnachfrage der Geschäftskunden sollte laut der BREKO-Studie als weitere Motivation zum flächendeckenden Breitbandausbau gesehen werden.

\subsection{Stand des Breitbandausbaus in Sachsen}

Im Folgenden wird die Summe aller leitungsgebundenen Technologien für Übertragungsgeschwindigkeiten über 50 Mbit/s als Momentaufnahme im J ahr 2019 dargestellt. Der Breitbandausbau wird in dieser Analyse ausschließlich als Netzinfrastruktur betrachtet. Die weiteren Netzebenen, wie Netzdienstleistungen oder das Infrastrukturmanagement, werden nicht weiter untersucht. Fokussiert wird außerdem der Teilabschnitt des terrestrischen Breitbands, welcher sich zwischen dem Hauptnetz (Backbone) und dem Haushaltsanschluss befindet. ${ }^{10}$

Abbildung 1 ordnet den Freistaat Sachsen im Vergleich zu den übrigen Flächenländern ein. Die Länder sind nach Raumtypen für die Haushalte sowie Gewerbestandorte gegliedert. Die Vertikale zeigt den Anteil an angeschlossenen Haushalten bzw. Gewerbestandorten. Es wird deutlich, dass alle Flächenländer hinsichtlich der städtischen Regionen auf einem Niveau von über 90 \%Breitbandverfügbarkeit mit einer Geschwindigkeit von 50 Mbit/ s und höher liegen. Als einzige Ausnahme ist hier Sachsen-Anhalt mit gut 88 \% zu nennen. Im halbstädtischen Bereich liegen alle ostdeutschen Flächenländer - mit Ausnahme von Brandenburg - unter dem bundesdeutschen Durchschnitt. Für den ländlichen Raum rangiert Sachsen mit ca. 55 \% an vorletzter Stelle und wird nur von Mecklenburg-Vorpommern unterboten. Die Gewerbestandorte werden in Sachsen mit 80 \% ebenfalls nur unterdurchschnittlich mit Internetgeschwindigkeiten von über $50 \mathrm{Mbit} / \mathrm{s}$ versorgt. Im Vergleich mit den ostdeutschen Flächenländern wird deutlich, dass Sachsen im halbstädtischen Raum das Schlusslicht bildet. Auch im ruralen Bereich fällt Sachsen hinter Thüringen und Brandenburg zurück.

10 Für eine vertiefte ökonomische Einordnung des Breitbandinternets als Netzökonomie siehe Mengs/ Günther (2018). 
Abbildung 1: Breitbandverfügbarkeit der Haushalte und Gewerbestandorte in Flächenländern ( $\geq 50$ Mbit/s, alle Technologien) nach Ländern im J ahr 2019

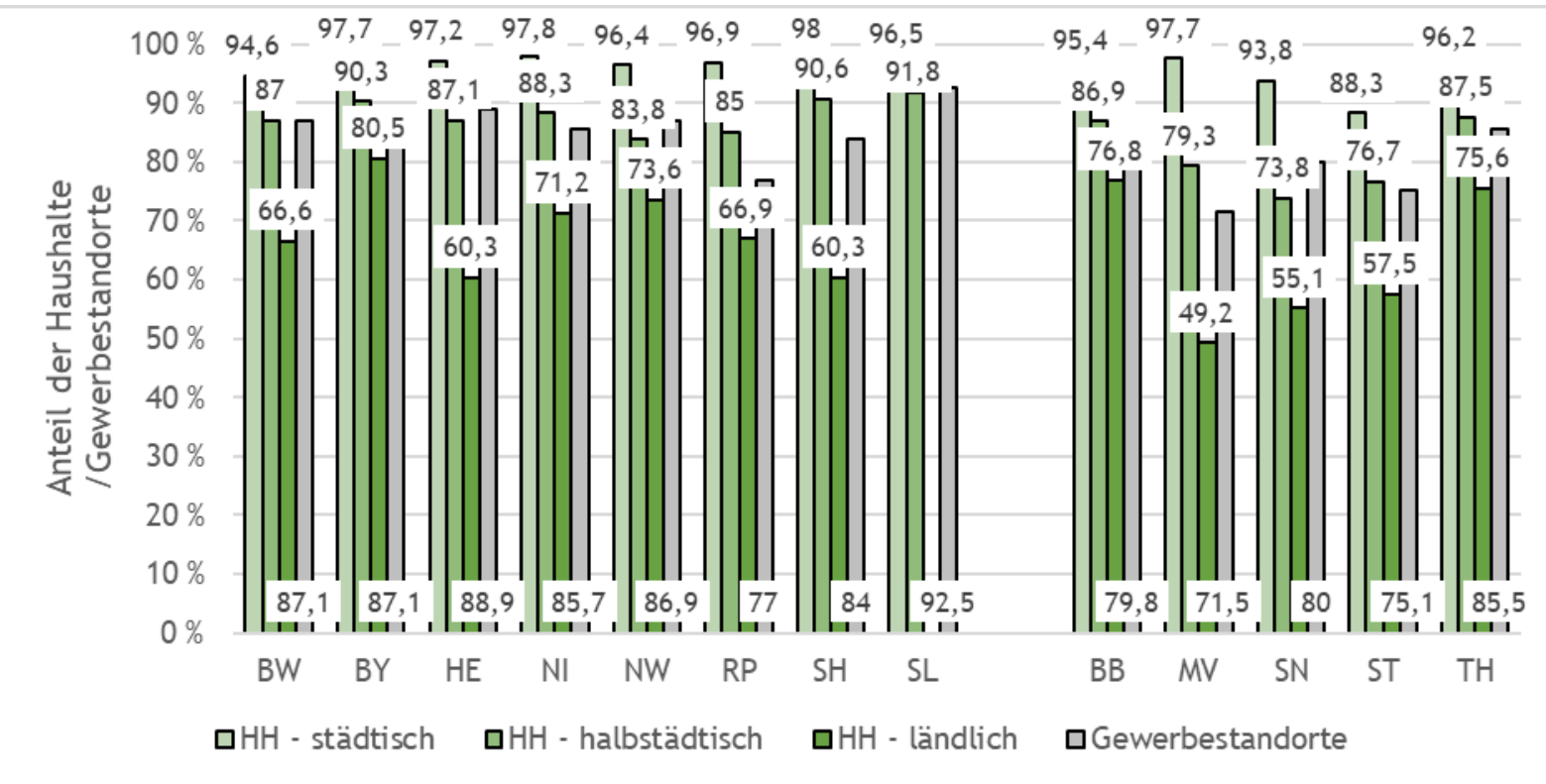

Quelle: Eigene Darstellung; eigene Berechnung; Daten: BMVI (2019).

Für den Netzausbau werden unterschiedliche Übertragungstechnologien genutzt. Dabei wird zwischen DSL/ VDSL ([Very High Speed] Digital Subscriber Line), CATV (Cable Television) und FTTB/H (Fiber To The Building/ Home) unterschieden. ${ }^{11}$ Letztere Abkürzung steht für das reine Glasfaserkabel, das vor dem Hintergrund der Gigabitziele als vorrangige Technologie gilt. Der CATV-Anschluss beschreibt das Kabelnetz, das für TV- und Radioübertragungen installiert wurde, und weitestgehend aus Koaxial- und/ oder Glasfaserkabeln besteht, wohingegen das DSL-Netz ausschließlich auf Kupferkabeln basiert.

Wie es in der Bundesrepublik flächendeckend typisch ist, haben nahezu alle Haushalte in Sachsen einen Zugang zu einem DSL-Anschluss. Hinsichtlich der Nutzung von TV-Kabelanschlüssen für das Breitbandinternet bewegen sich die sächsischen Haushalte im bundesweiten Vergleich mit 55,2 \% im Mittelfeld, während sie diese Technologie in Bezug auf die ostdeutschen Flächenländer überdurchschnittlich stark nutzen. Mit 9,5\% der Haushalte, die einen Glasfaseranschluss besitzen, lag Sachsen im J ahr 2019 im bundesweiten Vergleich hinter den Flächenländern Nordrhein-Westfalen (10,9\%), Bayern (14,2\%) und Schleswig-Holstein (22,5\%). Demzufolge ist es auch für Sachsen noch ein langer Weg bis zur "flächendeckende[n] Verfügbarkeit von gigabitfähiger Infrastruktur" ${ }^{12}$. Wie in den folgenden Abbildungen gezeigt wird, hat Sachsen den städtischen Raum und Gewerbestandorte betreffend einen vergleichsweisen fortschrittlichen Stand des gigabitfähigen Breitbandinternets erreicht.

11 Vgl. BMWI (2019).

12 Bundesregierung (2020). 
Abbildung 2: Breitbandverfügbarkeit der Haushalte in Flächenländern nach leitungsgebundenen Technologien im J ahr 2019

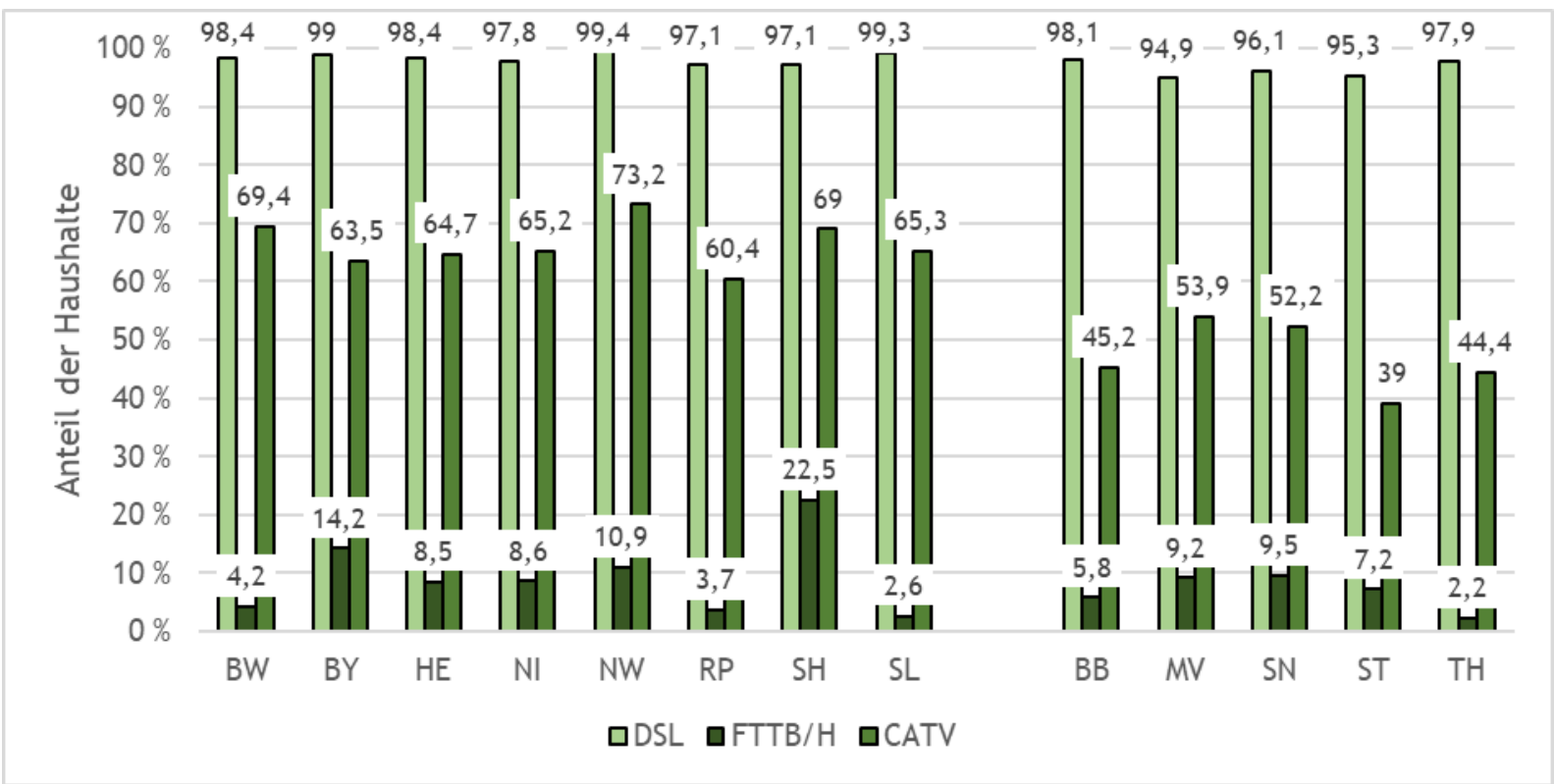

Quelle: Eigene Darstellung; eigene Berechnung; Daten: BMVI (2019).

Abbildung 3 illustriert ebenfalls die Technologien des Internetanschlusses nach den FlächenIändern sortiert, jedoch hier für die Gewerbestandorte. Es zeichnet sich ein ähnliches Bild: Fast die Gesamtheit der Gewerbestandorte hat einen DSL-Zugang. Für Gewerbestandorte in Sachsen liegt der Anteil der CATV-Technologie von 45, 5 \% im Jahr 2019 etwas unter dem Wert der Haushalte. Die Anschlüsse mit FTTB/ H-Anbindung sind in Sachsen (7,1\%) nur leicht niedriger als im Bereich der Haushalte, was im Einklang mit den Beobachtungen für die gesamte Bundesrepublik steht. In einigen Flächenländern hingegen sind Gewerbestandorte nur zu sehr geringen Anteilen mit Glasfaserinfrastruktur ausgestattet. 
Abbildung 3: Breitbandverfügbarkeit der Gewerbestandorte in Flächenländern nach leitungsgebundenen Technologien im J ahr 2019

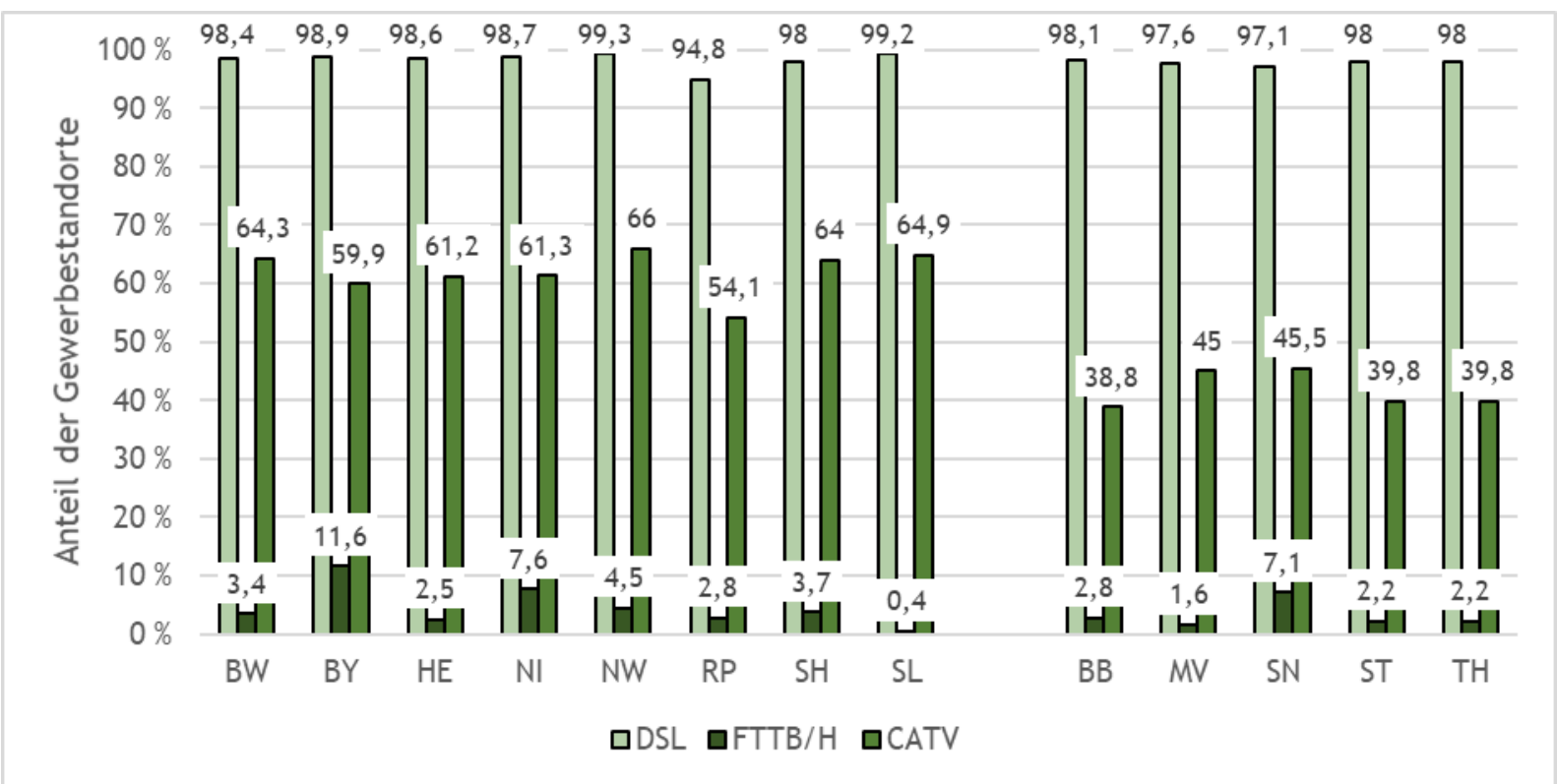

Quelle: Eigene Darstellung; eigene Berechnung; Daten: BMVI (2019).

Der Freistaat Sachsen wird im Folgenden in den Mittelpunkt der Betrachtung gerückt. Dafür ändert sich die Darstellung in Abbildung 4 und zeigt die verschiedenen Übertragungsgeschwindigkeiten von über 16 Mbit/s bis über 1000 Mbit/s (Gigabit). Für höhere Datentransferraten sinkt der Anteil versorgter Haushalte stetig. Der Unterschied zwischen städtischen und ländlichen Raum steigt von 31,9 Prozentpunkten für Bandbreiten von über 16 Mbit/s auf 67, 6 Prozentpunkte für Bandbreiten von über 400 Mbit/s. Hingegen ändern sich die Anteile für den städtischen Raum und die Gewerbestandorte nicht wesentlich für geringere Übertragungsgeschwindigkeiten. Die graphische Darstellung zeigt unverkennbar, dass höhere Datenraten insbesondere in nicht-städtischen Gebieten unterrepräsentiert sind. Im ländlichen Raum besitzen nur 36, 2 \% der Haushalte einen Anschluss mit einer Bandbreite von über 100 Mbit/s. Auch das Gewerbe fällt bei einer Übertragungsgeschwindigkeit von über $50 \mathrm{Mbit} / \mathrm{s}$ deutlich unter die Marke von 80 \% Gigabitfähige Leitungen liegen bisher im städtischen Bereich bei 58,9\%der Haushalte und zu 32,3\% an Gewerbestandorten vor. 
Abbildung 4: Breitbandverfügbarkeit der Haushalte und Gewerbestandorte in Sachsen (alle Technologien) nach Übertragungsgeschwindigkeiten im J ahr 2019

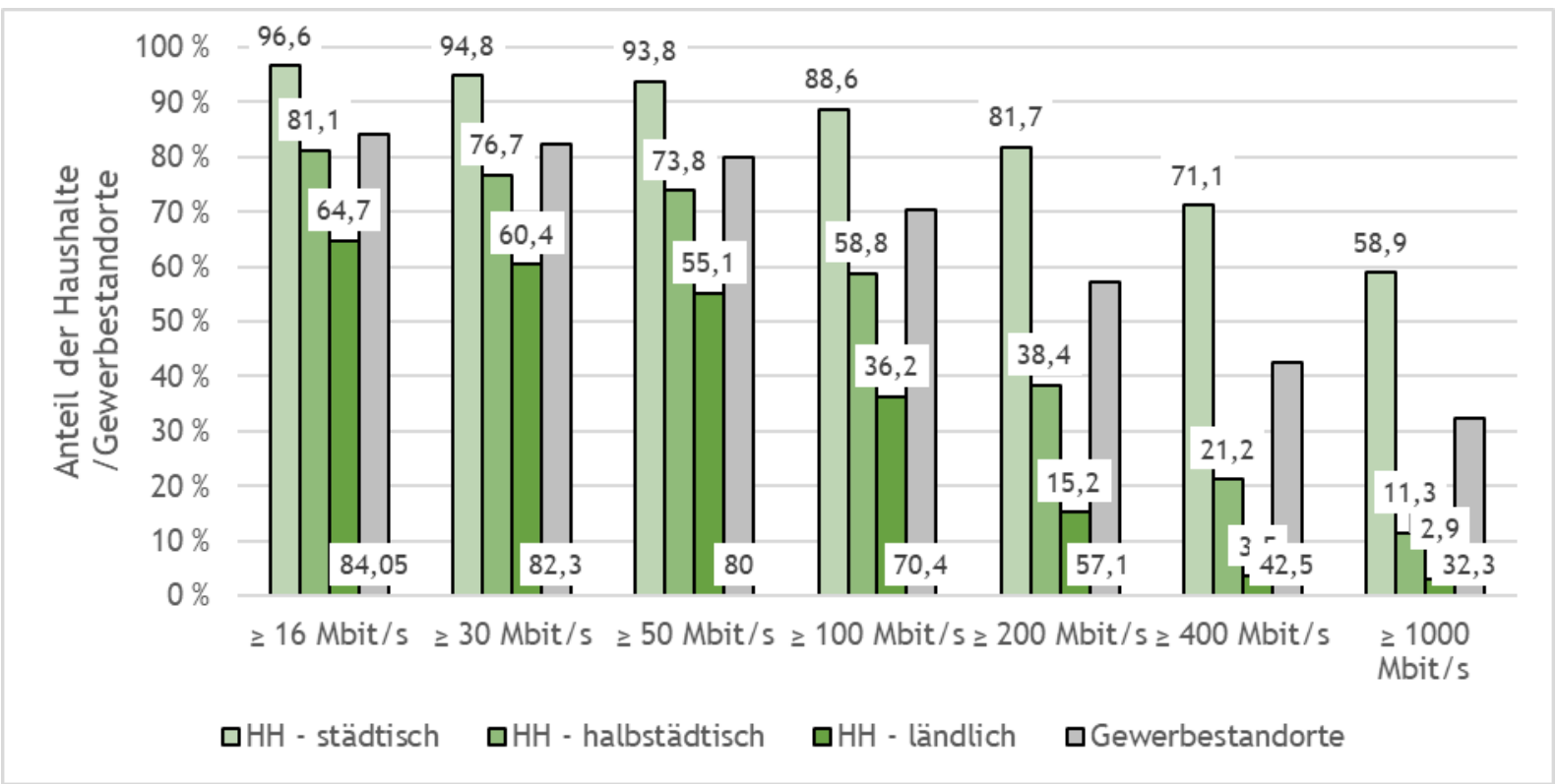

Quelle: Eigene Darstellung; eigene Berechnung; Daten: BMVI (2019).

Abbildung 5 und Abbildung 6 stellen die regionale Verteilung der Breitbandverfügbarkeit von über 50 Mbit/s für die Haushalte bzw. die Gewerbestandorte im Freistaat Sachsen dar.

Abbildung 5: Karte der Breitbandverfügbarkeit für Privathaushalte in Sachsen ( $\geq 50 \mathrm{Mbit} / \mathrm{s}$, alle Technologien)

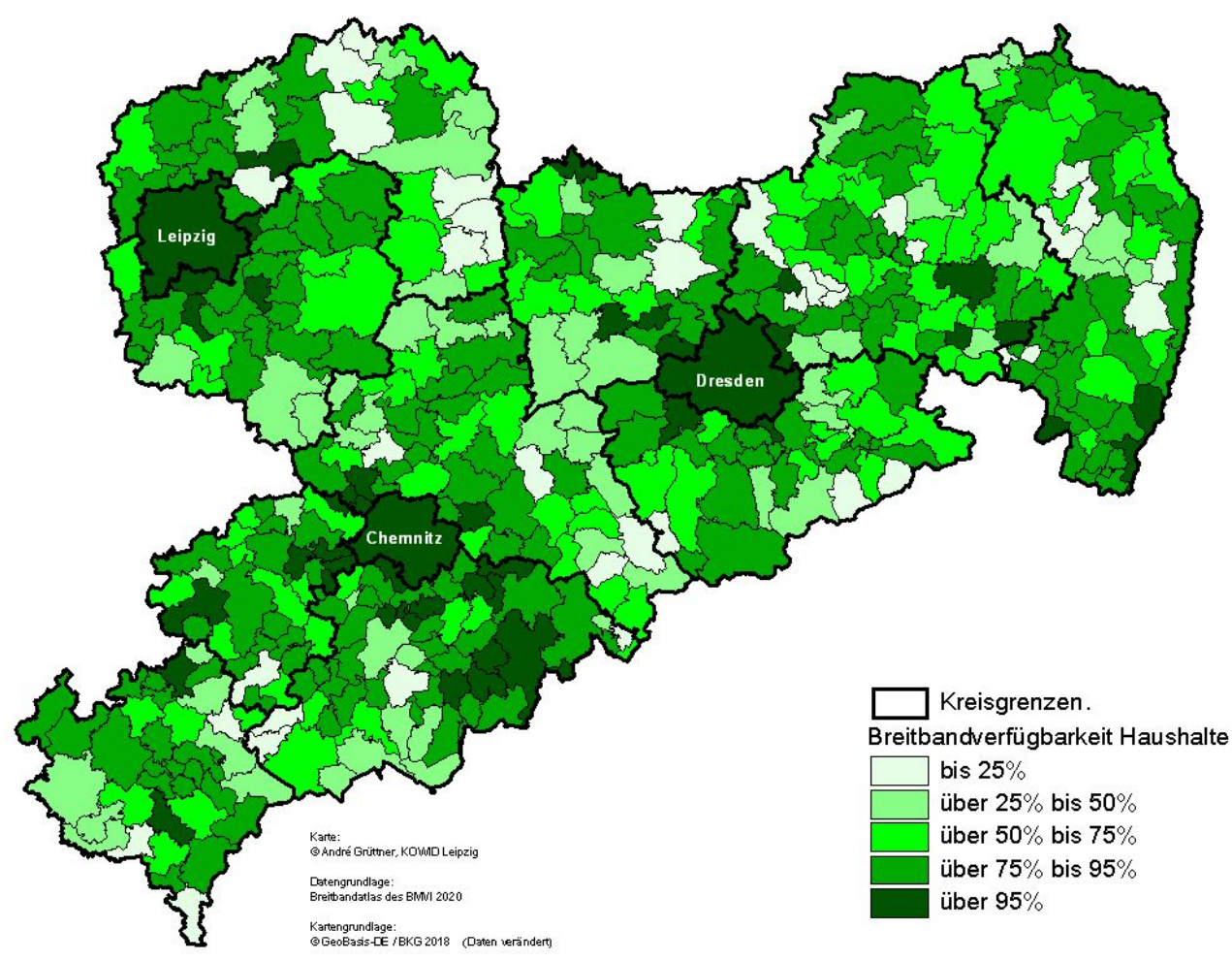

Quelle: Eigene Darstellung; eigene Berechnung; Daten: BMVI (2019). 
Wie bereits anhand der Balkendiagramme gezeigt, sind die Haushalte im städtischen Raum daher die drei kreisfreien Städte und teilweise auch das jeweilige Umland - durchgängig zu über 95 \% mit Übertragungsgeschwindigkeiten von über 50 Mbit/ s versorgt. Darüber hinaus sind insbesondere die Haushalte der Gemeinden im Erzgebirgskreis rund um Annaberg-Buchholz mit diesen Datentransferraten ausgestattet. In ländlichen Landesteilen sind größtenteils 50 \% der Haushalte mit Breitbandgeschwindigkeiten von bis zu 50 Mbit/s ausgestattet. Doch auch in den ländlichen Regionen gibt es einige besser versorgte Kommunen, in denen bis zu $75 \%$ der Haushalte $50 \mathrm{Mbit} / \mathrm{s}$ aufweisen.

Für die Gewerbestandorte zeichnet sich ein ähnliches Bild ab. Auffällig ist, dass es in den kreisfreien Städten nur gelungen ist, mindesten 75 \% der wirtschaftlich genutzten Flächen zu versorgen. Demgegenüber ist das Gewerbe in einigen ländlichen Gemeinden besser versorgt als die dortigen Haushalte, wie zu sehen im Raum Chemnitz und in der Niederlausitz.

\section{Abbildung 6: Karte der Breitbandverfügbarkeit für Gewerbestandorte in Sachsen ( $\geq 50$} Mbit/ s, alle Technologien)

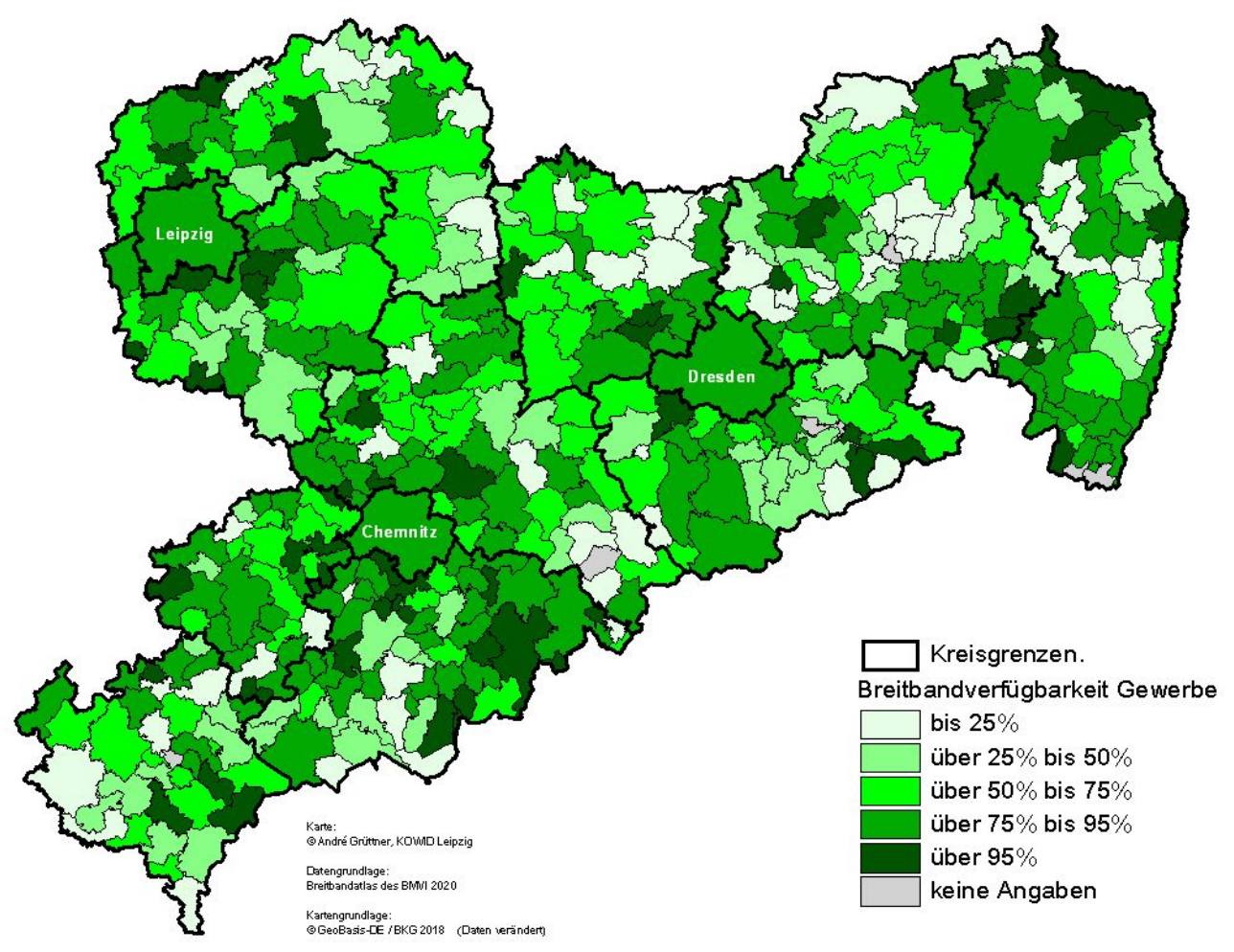

Quelle: Eigene Darstellung; eigene Berechnung; Daten: BMVI (2019). 


\section{Die Rolle der Tiefbaumaßnahmen für den Breitbandausbau}

Obwohl die Telekommunikationsanbieter per se für den Breitbandausbau zuständig sind, kann bei Marktversagen die öffentliche Hand eingreifen, um die Versorgung ihrer Bürger und Unternehmen voranzutreiben. In Sachsen ist der von Bund und Land geförderte Ausbau eine kommunale Aufgabe. ${ }^{13}$ Dabei haben die Kommunen nicht immer die finanziellen und personellen Kapazitäten, die Ausbauproj ekte in der gewünschten Form zu stemmen. Dort, wo der marktgetriebene Ausbau nicht erfolgt, stellt der Breitbandausbau somit neben den kommunalen Bautätigkeiten und sonstigen Dienstleistungen eine weitere Belastung der kommunalen Verwaltung dar. Nicht zuletzt ist die Verfügbarkeit von Infrastrukturen, wie auch dem Hochgeschwindigkeitsinternet, als ein Standortfaktor für Bürgerinnen und Bürger sowie Unternehmen zu sehen. Daher gilt eine gute Breitbandverfügbarkeit als eine wesentliche Determinante für das wirtschaftliche Wohlergehen der Kommunen.

Viele Herausforderungen, die in Infrastrukturprojekten und insbesondere bei Tiefbaumaßnahmen immer wieder auftreten, sind auch für den Breitbandausbau von Relevanz. So ist die Fördermittelproblematik ein wiederkehrendes Thema in Infrastrukturprojekten. Auch die Ausnutzungsdichte sowie die räumliche Streuung (sog. Zersiedlung) spielt für Tiefbaumaßnahmen eine wichtige Rolle. Es entstehen positive Agglomerationseffekte in Ballungsgebieten, die als kostensenkende ökonomische Skaleneffekte in der Infrastrukturbeschaffung und -bewirtschaftung bedeutsam sind. So geraten insbesondere kleine Kommunen, die keinen Ballungsvorteil nutzen können und die zusätzlich geringen Personalkapazitäten aufweisen oder unter einem hohen Schuldenstand leiden, an die Grenzen des für sie Machbaren. Als hilfreich stellen sich dem gegenüber (institutionalisierte) Formen der interkommunalen Zusammenarbeit heraus.

Weiterhin schafft die integrierte Planung von öffentlichem Tiefbau und Breitbandausbau neue Themenfelder, die im kommunalen Kontext eine hohe Aktualität haben. Tiefbaumaßnahmen im Bereich der Netzinfrastrukturen können ganze Straßenzüge, Wohnquartiere oder Gewerbegebiete über einen beträchtlichen Zeitraum in der gewohnten Versorgung einschränken und so maßgeblich die gewohnte Qualität von öffentlich bereitgestellten Leistungen reduzieren. Darüber hinaus können weitere Kosten entstehen, sofern verschiedene Betreiber für den Breitbandausbau und weitere Infrastrukturen, wie die Energieversorgung und Siedlungswasserwirtschaft, sich in ihren Arbeiten nicht koordinieren und im schlechtesten Fall gegenseitig behindern. Daraus können Synergien generiert werden, sodass eine verbesserte Koordination im Tiefbau als ein maßgeblicher Problemlösungsansatz gelten kann.

13 Für nähere Informationen dazu siehe Mengs/ Günther (2018), S. 9-12. 
Um dies sicherzustellen, kommt den Bau- und Hauptämtern der Städte und Gemeinden eine Schlüsselrolle zu: Sowohl die eigenen Baumaßnahmen, als auch jener Dritter sind zu koordinieren. Hierbei gilt es, nicht nur die eigentliche frühzeitige Koordination, die bereits in der Planungsphase zu erfolgen hat, im Blick zu behalten, sondern auch die zur Verfügung stehende Informationsbasis zu verbreitern. Es besteht ein deutlicher Unterschied, ob eine zentrale Datenbank einen Überblick über die bereits durchgeführten, laufenden und geplanten Tiefbaumarbeiten bereitstellt, insbesondere im Rahmen einer bedienungsfreundlichen digitalen Anwendung, wie einem Geoinformationssystem (GIS), oder ob die relevanten Informationen analog zur Verfügung stehen oder gar dezentral in den Ämtern vorliegen. Zudem gilt es, nicht nur scheinbar originäre Informationen in digitalisierter Form zentral vorzuhalten, sondern z. B. auch ergänzende Informationen, wie Angaben zu Wasser- und Naturschutzgebieten, die situativ ergänzend hinzugezogen werden können.

Infolge einer integrierten Planung werden die Maßnahmen transparent und koordinierungsfähig. Im besten Fall werden Baumaßnahmen im Tiefbau innerhalb der Kommune oder gar im Rahmen von interkommunaler Kooperation gebündelt durchgeführt. Die Nutznießer sind in diesem Fall neben den Kommunen unter anderem die Telekommunikationsunternehmen, die (Energie-)Versorgungsbetriebe und der ÖPNV. Überdies können durch einen gebündelten Ausbau Umwelt- und Lärmbelastungen gesenkt werden.

Welche Hindernisse mit Blick auf eine stärker vernetzte Verwaltung im Sinne einer Tiefbaukoordination bestehen, ist mithilfe der nachfolgend dargestellten Kommunalbefragung untersucht worden. Außerdem kann in Verbindung mit weiteren Abfragen zum Breitbandausbau auf mögliche Zusammenhänge geschlossen werden. Inwieweit und in welcher Form eine neue Dateninfrastruktur zielführend ist, kann im Rahmen dieser Studie jedoch nur angerissen werden. 


\section{Studienergebnisse}

Im dritten Kapitel werden die Studienergebnisse, die auf der Befragung und anderen Statistiken beruhen, ausführlich dargestellt. Zuerst erfolgt eine Beschreibung der erhobenen Daten, woraufhin im zweiten Schritt eine explorative Darstellung der Studienergebnisse mithilfe der Befragungsergebnisse und weiterer Daten durchgeführt wird. Dabei werden verschiedene Auswertungsmethoden angewandt.

\subsection{Deskriptive Statistik}

Insgesamt nahmen 69 von den 322 angeschriebenen sächsischen Kommunen an der Befragung teil. Daraus ergibt sich eine Rücklaufquote von 21,4\% Da der Befragungszeitraum (erste J ahreshälfte 2020) in die Zeit des ersten Lockdowns infolge der Corona-Pandemie fiel, war es weniger Kommunen als gewöhnlich möglich, an der Erhebung teilzunehmen. Dennoch ist die Rücklaufquote vergleichbar mit bisherigen Umfragen des KOMKIS und wird als hinreichend groß eingeschätzt.

Von den Rückläufen wurden 42 Fragebögen für die Auswertung verwendet. Die Differenz ergibt sich aus Fragebögen, die nur teilweise ausgefüllt wurden oder unplausible Antworten enthielten. Die Stichprobe enthält drei Landkreise und 39 kreisangehörige Städte oder Gemeinden. Die Tatsache, dass nur eine geringe Fallzahl der Landkreise vorliegt und sich die Aufgabenbereiche der Landkreise und Städte bzw. Gemeinden unterscheiden, führt aus methodischen Gründen dazu, dass nur die kreisangehörigen Kommunen in Kapitel 3.2 näher analysiert werden.

\section{Abbildung 7: Befragte Kommunen nach Kommunaltypus}

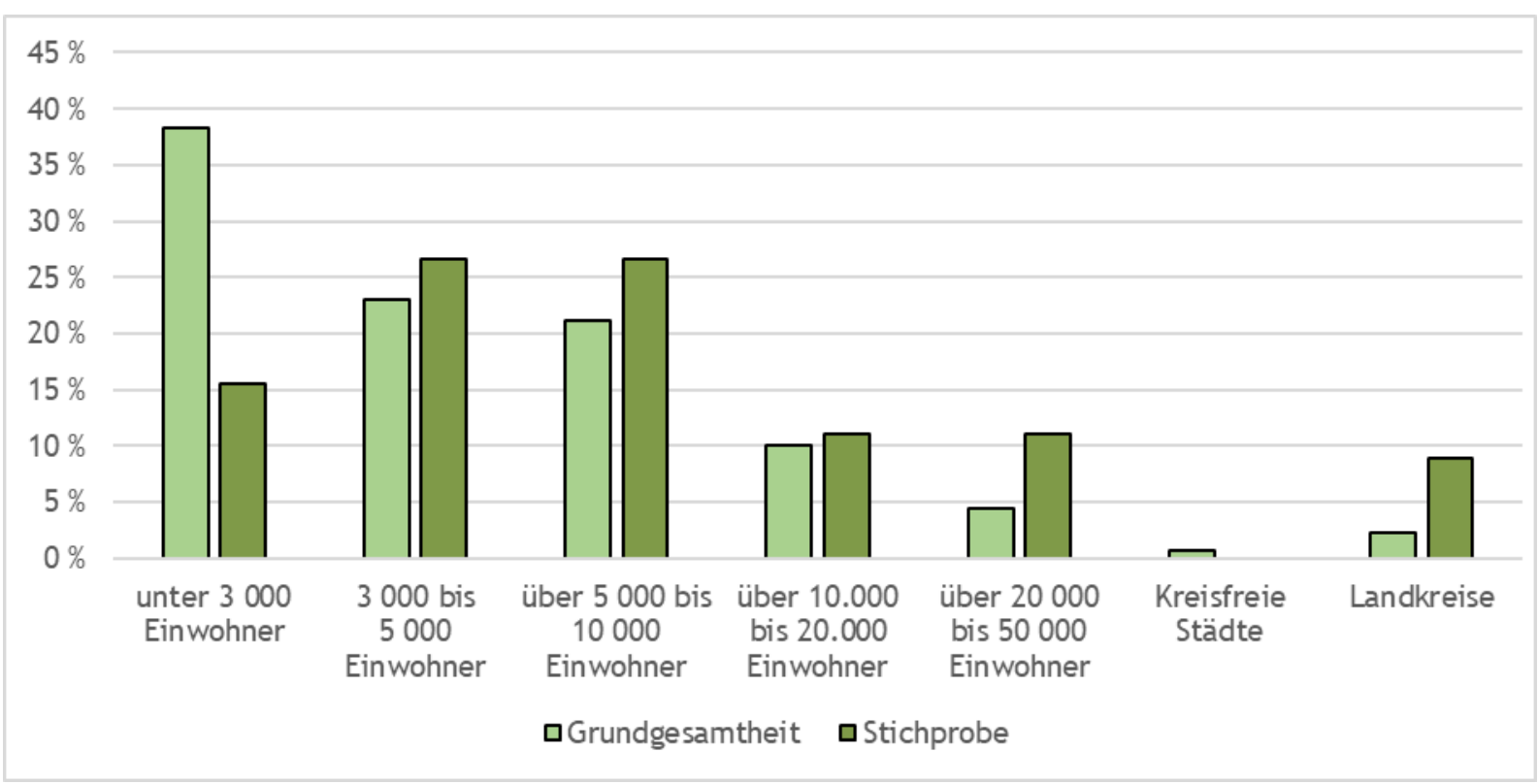

Quelle: Eigene Darstellung; eigene Berechnung. 
Sofern die übliche Unterteilung der kreisangehörigen Städte und Gemeinden in Sachsen nach Einwohnerklassen vorgenommen wird, zeigt sich das Bild wie in Abbildung 7dargestellt. In hellgrün wird der relative Anteil der Kommunen in der Gruppe an der Grundgesamtheit und in dunkelgrün der Anteil in der Stichprobe dargestellt. Die kreisfreien Städte haben nicht an der Befragung teilgenommen. Für die Kommunen mit 3000 bis 5000 Einwohnern liegen mit 29 \% (Grundgesamtheit: $23 \%$ ) die meisten Antworten vor. Auf ebenfalls 29 \% (Grundgesamtheit: $21 \%$ beläuft sich der Rücklauf der Kommunen mit 5000 bis 10000 Einwohnern. In einem ähnlichen Verhältnis sind die Kategorien 10000 bis 20000 Einwohner (Stichprobe: $12 \%$ Grundgesamtheit: $10 \%$. Etwas über dem Anteil in der Grundgesamtheit von 4 \%liegt der Anteil der Städte mit 20000 bis 50000 mit 10 \%Anteil an der Stichprobe. In der Kategorie der Gemeinden unter 3000 Einwohnern ist der Anteil der Kommunen an der Stichprobe wesentlich geringer als der wahre Anteil im Freistaat Sachsen. Laut einiger Rückmeldungen der Kommunen waren diese zum Zeitpunkt der Befragung so sehr überlastet, dass sie nicht an der Umfrage teilnehmen konnten. Im nächsten Kapitel wird versucht, der möglicherweise auftretenden Verzerrung an dieser Stelle zu begegnen, um die besonderen Bedarfe kleinerer Kommunen Rechnung zu tragen.

Die Tabelle 1 präsentiert für die abgefragten Variablen die Kennzahlen Mittelwert, Median Standardabweichung, Minimum und Maximum. Der Mittelwert ist als arithmetischen Mittel berechnet. Der Median teilt den Datensatz in zwei gleich große Hälften, daher liegen 50 \% der Datenpunkte unter dem Medianwert und 50 \% der Datenpunkt über diesem. Der Modus gibt die am häufigste gegebene Antwort an. Zusätzlich gibt die Standardabweichung ein Maß für die Streuung um den Mittelwert an. Das Minium und das Maximum zeigen den niedrigsten bzw. den höchsten angegeben Wert im Datensatz an. Die deskriptive Einordnung macht deutlich, dass sich einzelne Antworten für die jeweiligen Fragen teilweise deutlich voneinander unterscheiden. 
Tabelle 1: Deskriptive Statistik der metrischen Merkmalsträger

\begin{tabular}{|c|c|c|c|c|c|}
\hline Variable & Mittelwert & Median & Std.-Abw. & Min. & Max. \\
\hline Einwohnerzahl & 29101 & 5801 & 74388 & 1800 & 322099 \\
\hline Wohngebäudezahl & 2816 & 2282 & 2647 & 200 & 8700 \\
\hline Kassenkredite in $€$ je EW & 28 & 0 & 33 & 0 & 30 \\
\hline investive Schulden in $€$ je EW & 128 & 381 & 53 & 0 & 44 \\
\hline Gesamtfläche & 14631 & 3640 & 40481 & 550 & 211685 \\
\hline Wohnbaufläche & 267 & 250 & 163 & 24 & 613 \\
\hline Verkehrsfläche & 423 & 121 & 1159 & 12 & 5167 \\
\hline Bauamt-VZÄ & 5,5 & 3 & 5,7 & 1 & 20 \\
\hline Hauptamt-VZÄ & 3,3 & 3,2 & 1,3 & 2 & 5 \\
\hline $\begin{array}{l}\text { Länge der Bundes- und Landes- } \\
\text { straßen }\end{array}$ & 59 & 16,8 & 135,9 & 1,9 & 493 \\
\hline Länge der Kreisstraßen & 52,3 & 9,9 & 137,1 & 1,1 & 575 \\
\hline Länge der Gemeindestraßen & 171,7 & 48,4 & 424,8 & 22,8 & 1705,2 \\
\hline $\begin{array}{l}\text { prozentualer Anteil der Haus- } \\
\text { halte mit } 50 \mathrm{Mbit} / \mathrm{s}\end{array}$ & 61,7 & 74 & 31,6 & 0 & 95 \\
\hline $\begin{array}{l}\text { prozentualer Anteil der Gewer- } \\
\text { befläche mit } 50 \mathrm{Mbit} / \mathrm{s}\end{array}$ & 63,7 & 80 & 38,7 & 0 & 100 \\
\hline $\begin{array}{c}\text { Anzahl Instandhaltungsmaßnah- } \\
\text { men Tiefbau }\end{array}$ & 4 & 3 & 4 & 0 & 15 \\
\hline $\begin{array}{c}\text { Anzahl Neubaumaßnahmen } \\
\text { Tiefbau }\end{array}$ & 2 & 1 & 2 & 0 & 7 \\
\hline
\end{tabular}

Quelle: Eigene Darstellung; eigene Berechnungen.

\subsection{Explorative Statistik}

Die explorative Statistik gibt ebenfalls Auskunft über die Studienergebnisse der Befragung, jedoch geht sie methodisch einen Schritt weiter. Dabei werden die Ergebnisse gemäß dem Aufbau der Befragung wiedergegeben. ${ }^{14}$ Dahingehend wird im nachfolgenden Teil A zuerst auf die allgemeinen Angaben eingegangen, während in Teil B die Tiefbaukoordination im Fokus steht. Teil $\mathrm{C}$ behandelt die Ergebnisse zu den Fragen bezüglich des Breitbandausbaus. Hierbei sind Techniken der Hochrechnung und Gewichtung zum Einsatz gekommen. Für eine konzeptionelle Beschreibung der statistischen Methoden kann der Anhang 5.1 konsultiert werden. Im Rahmen der Hochrechnung wurden die Ergebnisse der Stichprobe mit der bekannten Einwohnerverteilung der Kommunen in Sachsen, differenziert nach Einwohnergrö-

14 Im Anhang unter 5.2 ist der Fragebogen, welcher Grundlage der in diesem Abschnitt folgenden Ergebnisdarstellung ist, dokumentiert. 
ßenklassen extrapoliert. Diese Methode fand in dem Bereich Anwendung, in dem keine öffentlich zugänglichen Daten vom Statistischen Landesamt, aufbereitet nach Einwohnergrößenklassen, berichtet werden.

Die Ergebnisse für die Tiefbaukoordinierung und den Breitbandausbau sind für die einzelnen Merkmalsausprägungen, sofern möglich und theoretisch begründet, gewichtet worden. Hierdurch können Verzerrungen aufgrund des Studienrücklaufs ausgeglichen werden. ${ }^{15}$ Somit sind, neben der ungewichteten Darstellung der Ergebnisse, auch jeweils gewichtete Ergebnisse dargestellt, die über die eigentliche Einwohnergewichtung hinausgehen. Die zusätzlichen Gewichtungsformen sind immer dann ergänzend dargestellt, sofern es signifikante Unterschiede zur Einwohnergewichtung gibt.

\subsubsection{Teil A: Allgemeine Angaben}

Nachfolgend werden die Ergebnisse aus dem ersten Teil der Befragung für die weiterführende Analyse herangezogen. Wie Abbildung 8 zeigt, variiert der Bestand an Wohngebäuden je Einwohner zwischen den Einwohnerklassen: So nimmt die Anzahl an Wohngebäuden je Einwohner nach Gemeindegrößenklasse kontinuierlich ab. Dies begründet sich im ungleich verteilten Anteil an Ein- und Zweifamilienhäusern: In den Großstädten sind etwa 59 \% Einund Zweifamilienhäuser, wohingegen in Kommunen mit weniger als 3000 Einwohner über 91 $\%$ an Ein- und Zweifamilienhäuser an Wohngebäuden im Wohngebäudebestand existieren. Bezogen auf die Stichprobe lassen sich ähnliche Muster zur Grundgesamtheit festhalten.

15 Neben der Einwohnergewichtung wurden weitere Gewichtungsparameter eingesetzt, um zu evaluieren, ob auf Grundlage der Gewichtungsauswahl Verzerrungen erzeugt worden sind. Diese werden in den Grafiken aus Gründen der Übersichtlichkeit nicht durchgängig dargestellt, jedoch sofern signifikante Änderungen zu den anderen Gewichtungsdarstellungen vorliegen, im Text beschrieben. 
Abbildung 8: Wohngebäude je 100 Einwohner nach EW-Klasse

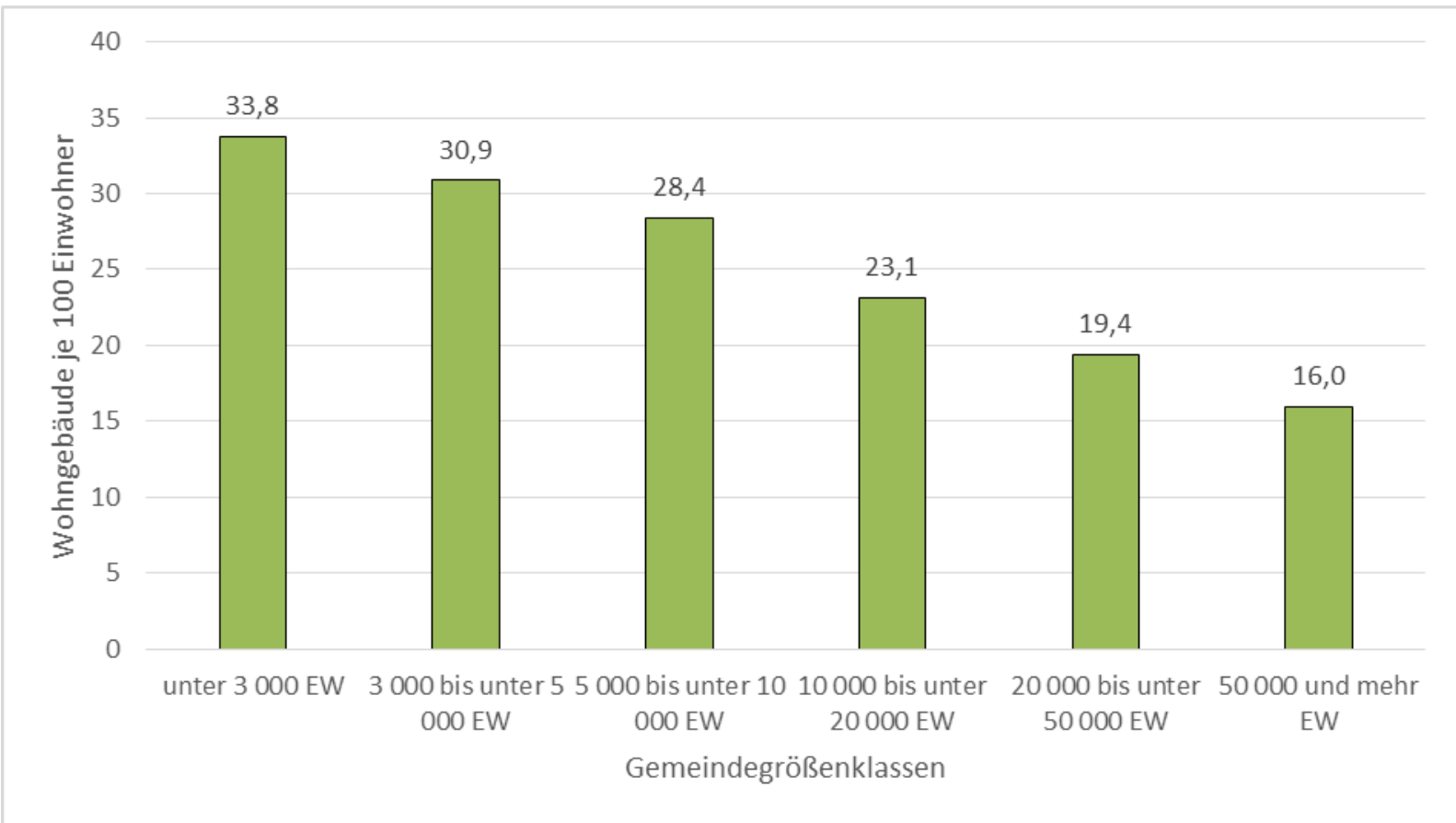

Quelle: Eigene Darstellung; eigene Berechnung; Daten: Landesamt für Statistik.

Eine Folge der ungleichen Verteilung an Wohngebäuden ist die divergierende Nachfrage nach Breitbandanschlüssen im privaten Bereich. So besteht an Ein- und Zweifamilienhäusern eine geringere Nachfrage, als bei Mehrfamilienhäusern, in denen mehr Einwohner wohnen. Hierdurch steigen die Anschlusskosten je Haushalt, was die privatwirtschaftlich ausgebauten Breitbandanschlüsse maßgeblich bestimmt. ${ }^{16}$ Deshalb wird der Schwerpunkt in dieser AnaIyse ebenfalls auf Indikatoren, die weniger die Breitbandnachfrage, sondern eher das Breitbandangebot erklären, aufbauen.

Ein ähnliches Bild ergibt sich bei der Betrachtung der Siedlungs- und Verkehrsfläche je Einwohner ${ }^{17}$, wie Abbildung 9 illustriert. In Bezug auf die Siedlungs- und Verkehrsfläche zeigt sich eine gleichartige Verteilung im Vergleich zur Abbildung 8. Interessanterweise wird jedoch bei der Betrachtung deutlich, dass der Anstieg hin zur EW-Klasse unter 3000 EW überproportional erfolgt. Dies ist nicht nur durch den zunehmenden Anteil an Ein- und Zweifamilienhäusern in der Wohnbebauung zu erklären, sondern auch durch einen enormen Anstieg der Verkehrsfläche. Die höhere Flächeninanspruchnahme je Einwohner, weist auf eine ineffizientere Siedlungsstruktur im ländlichen Raum, was maßgeblich einen marktbasierten Breitbandausbau behindert. Die in Abbildung 9 dargestellte Aufteilung nach EW-Klassen lässt

16 Vgl. Mengs/ Günther (2018).

17 Während die Verkehrsfläche diejenigen Flächen umfasst, die dem Straßen-, Schienen- und Flugverkehr dient, bezieht sich die Siedlungsfläche auf baulich geprägte Flächen, die vorwiegend dem Wohnen, Gewerbe und Industrie sowie der Erholung dienen. 
sich auch auf die Stichprobe übertragen: Kommunen mit weniger Einwohner weisen eine überproportional größere SuV-Fläche je Einwohner auf.

Abbildung 9: Siedlungs- und Verkehrsfläche je 1000 Einwohner nach EW-Klasse

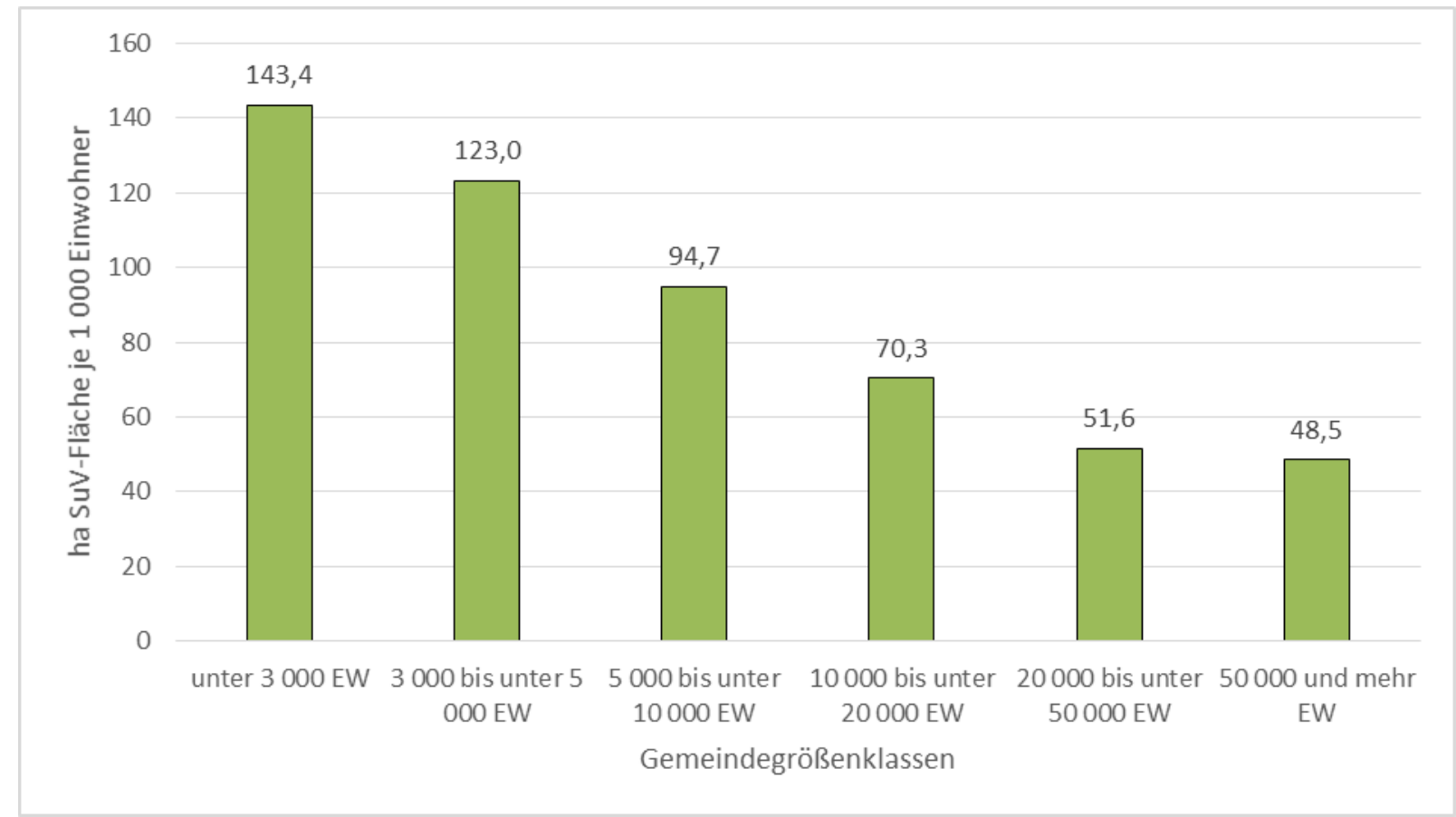

Quelle: Eigene Darstellung; eigene Berechnung, Daten: Landesamt für Statistik.

Um zu beurteilen, ob sich diese Beobachtung in eine korrespondierende Verteilung des Straßenbestandes übersetzt, ist ebenfalls nach den Anteilen der Bundes- und Landes-, der Kreissowie der Gemeindestraßen gefragt worden. Hierzu gibt Abbildung 10 weiterführende Informationen, wobei hier die Angaben zu den Straßentypen gemäß den EW-Klassen hochgerechnet wurden. Zudem erfolgt eine prozentuale Darstellung gemessen an dem gesamten Straßenbestand der jeweiligen Einwohnerklasse, um eine Vergleichbarkeit der Ergebnisse zu gewährleisten. 


\section{Abbildung 10: Hochgerechneter Straßenbestand nach Typus in km nach EW-Klassen}

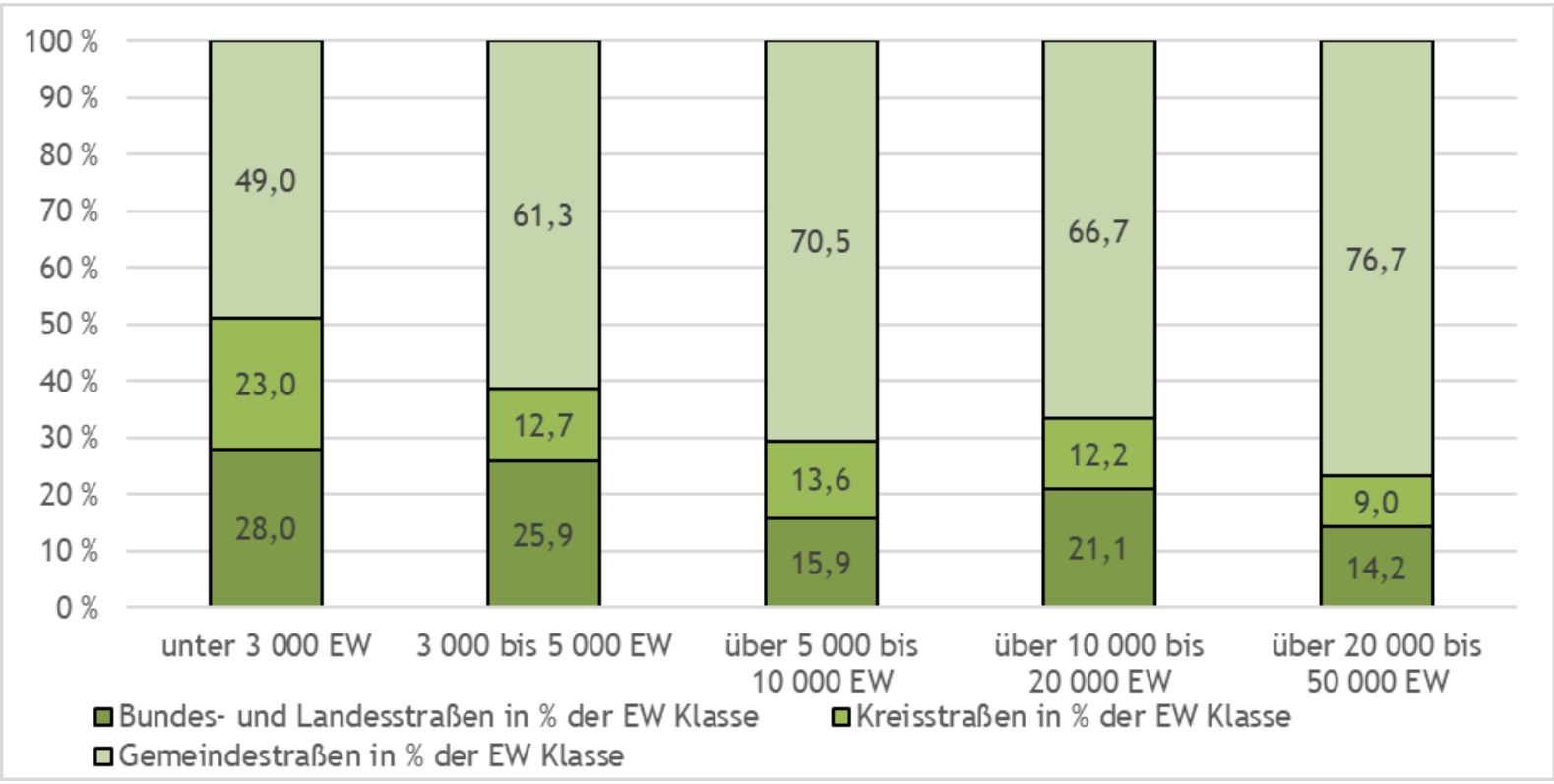

Quelle: Eigene Darstellung; eigene Berechnung, $\mathrm{N}=35$.

In der EW-Klasse unter 3000 bilden die Gemeindestraßen $49 \%$ Dieser Wert ist in der Betrachtung der EW-Klasse an sich zwar am größten, jedoch in den verbleibenden EW-Klassen wesentlich höher. Zudem zeigt sich, dass die unterste EW-Klasse im Vergleich zum gesamten Straßenbestand in ihrer EW-Klasse einen Bestand an Bundes- und Landesstraßen in Höhe von 28 \% besitzt. Dieser Wert ist in den verbleibenden EW-Klassen wesentlich geringer. Dasselbe Bild zeichnet sich für die Kreisstraßen fort. In der EW-Klasse 20000 bis 50000 machen die Gemeindestraßen 76,7 \% des gesamten Straßenbestandes in der Einwohnerklasse aus. ${ }^{18}$ Ein höherer Bestand an Straßen, die es zu unterhalten gilt, geht auch mit personellen Mehrbelastungen einher, die eine kommunale Verwaltung zu bewältigen hat. Genauso kann eine größere kommunale Fläche eine entsprechende Mehrbelastung zur Folge haben, sofern hieraus Verwaltungsdienstleistungen erwachsen, die zu erbringen sind. Abbildung 11 gibt daher die hochgerechneten VZÄ in den jeweiligen Bau- und Hauptämtern an. Hierbei gilt, dass immer dann die Anzahl der VZÄ im Hauptamt anzugeben war, sofern es kein eigenständiges Bauamt gibt.

18 Hierbei ist jedoch darauf hinzuweisen, dass keine Zuständigkeiten betrachtet werden, welche in Bender / Mengs (2020) nachvollzogen werden können. Vielmehr soll im Folgenden beleuchtet werden, dass Tiefbaumaßnahmen mit Breitbandausbau koordiniert werden kann und dies durch unterschiedliche Zuständigkeiten behindert werden könnte. 


\section{Abbildung 11: Hochgerechnete VZÄ (Bau-/ Hauptamt) je 1000 Einwohner nach EW-Klassen}

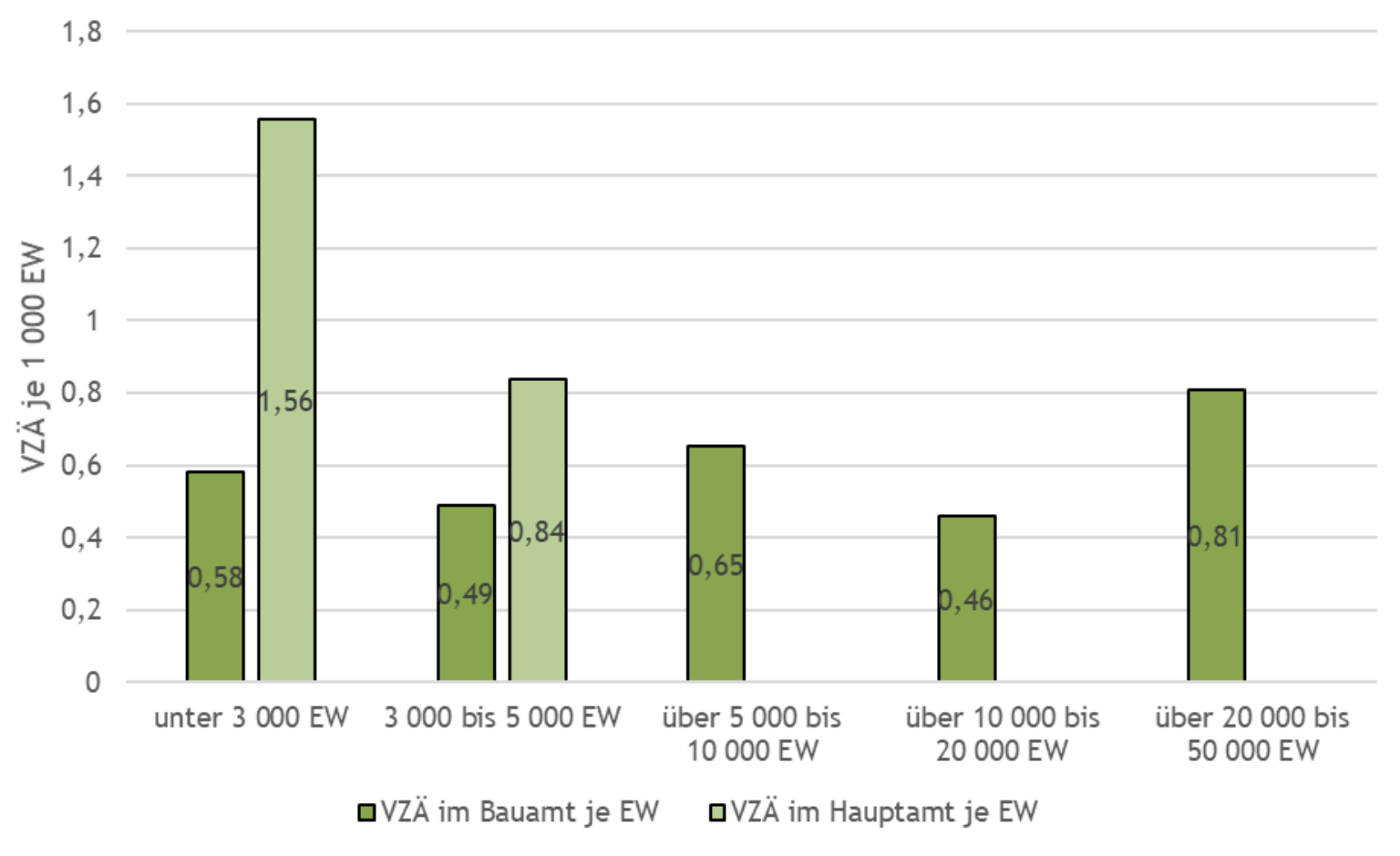

Quelle: Eigene Darstellung; eigene Berechnung, $N=35$.

Die Ergebnisse zeigen, dass ab einer EW-Größenklasse von 5000 bis 10000 EW die kommunalen Verwaltungen ihre Strukturen weitreichend differenziert haben. Für die beiden EWKlassen unter $3000 \mathrm{EW}$ und 3000 bis $5000 \mathrm{EW}$ stellen demnach eigenständige Bauämter eher die Ausnahme als die Regel dar, da diese gegenüber den Hauptämtern weniger stark ausgeprägt sind.

Für diese Kommunen ergibt sich damit, dass diese zwar einen hohen vielseitigen Straßenbestand zu unterhalten haben, jedoch im Vergleich über eine weniger differenzierte Bauverwaltung verfügen. Zwar ist festzustellen, dass die unterste EW-Klasse über einen hohen VZÄBestand je 1000 EW im Hauptamt verfügt, jedoch ist zu konstatieren, dass die Hauptämter nicht nur bauwirtschaftliche Tätigkeiten, sondern die gesamte kommunale Verwaltung abbilden. Vor diesem Hintergrund sind die Zahlen zu den Hauptämtern nur mit Vorsicht zu interpretieren, da diese neben den Aufgaben der Tiefbaukoordinierung anderweitige kommunale Verwaltungsleistungen zu erledingen haben.

Es ergibt sich daher für die unteren beiden EW-Klassen das Bild einer kommunalen Verwaltung, die mit hohen Straßenbeständen und teilweise hohen Flächenbelastungen konfrontiert ist, jedoch nur über eingeschränkte personelle Mittel verfügen.

Dies gilt in eingeschränkter Weise auch für die EW-Klasse 10000 bis $20000 \mathrm{EW}$. Hier zeigt sich, dass insbesondere im Vergleich zu den beiden EW-Klassen 5000 bis 10000 EW und 20000 bis $50000 \mathrm{EW}$ ein geringerer Bestand an VZÄ je $1000 \mathrm{EW}$ im Bauamt vorhanden ist, 
jedoch gerade im Vergleich zu der letztgenannten EW-Klasse die Flächenbelastung, wie auch die Kosten der Straßenbewirtschaftung, hoch ist.

Eine Lösungsoption zur Reduzierung der negativen Auswirkungen einer verminderten Personaldecke in den kommunalen Verwaltungen kann die verstärkte interkommunale Zusammenarbeit darstellen. Daher wurde nachfolgend erfragt, ob die Kommunen Mitglied in einem Verwaltungsverband oder einer Verwaltungsgemeinschaft sind. In der vorliegenden Stichprobe ist dies für 29,5\% aller Kommunen der Fall. Insgesamt 70,5 \% der Kommunen gaben an, weder Mitglied eines Verwaltungsverbandes, noch Teil einer Verwaltungsgemeinschaft zu sein. Darüber hinaus existieren weitere öffentlich-rechtliche und privatrechtliche Formen der interkommunalen Zusammenarbeit in Sachsen. Der Gesetzgeber erlaubt ebenfalls eine informelle Basis der Kooperation, beispielsweise als Arbeitsgemeinschaften oder Netzwerktreffen, auf die ggf. aufgebaut werden kann. ${ }^{19}$

Verwaltungsverbände zeichnen sich dadurch aus, dass benachbarte Gemeinden desselben Landkreises sich zusammenschließen, um Aufgaben zentral zu erbringen. Hierbei können einerseits Aufgaben vollständig auf den Verwaltungsverband übergehen und andererseits kommunale Aufgaben durch den Verband nach Weisung der Mitgliedskommunen erledigt werden. ${ }^{20}$ Hingegen zeichnet sich eine Verwaltungsgemeinschaft dadurch aus, dass eine Gemeinde als erfüllende Gemeinde die Aufgaben für weitere Gemeinden (beauftragende Gemeinden) übernimmt. Interkommunale Kooperationen, auch in rechtlich-institutionalisierter Form, beschränken sich in Sachsen meist auf die dezidierten Bereiche, wie Wasser/ Abwasser, Standesämter und Feuerwehren. Kooperation im Bereich der Bauhöfe werden zwar von Kommunen erwünscht, bilden in Sachsen aber eher die Ausnahme. ${ }^{21}$ Dennoch werden - relevant für den Kontext des Breitbandausbaus - die Aufgaben der vorbereitenden Bauleitplanung gemäß $§ 7$ Abs. 1 Nr. 2 SächsKomZG grundsätzlich an die Verwaltungsverbände bzw. die erfüllende Gemeinde übertragen.

Die beschriebenen strukturellen Rahmenbedingungen bilden im weitesten Sinne die Voraussetzungen für den Breitbandausbau. Daher ist im weiteren Verlauf erfragt worden, wie hoch der Anteil der leitungsgebundenen Breitbandverfügbarkeit von 50 Mbit/ s der privaten Haushalte und des Gewerbes ausfällt. Das vergangene Ziel der Bundesregierung, dass bis Ende 2018 alle Haushalte in Deutschland Anschlüsse mit einer Geschwindigkeit von mindestens 50 Mbit/s nutzen können, bildet an dieser Stelle den Richtwert.

19 Für eine umfassende Darstellung der rechtlichen Grundlagen von interkommunaler Zusammenarbeit in Sachsen siehe Kratzmann (2020), S. 11-16.

20 Siehe $\S 7$ SächsKomZG.

21 Vgl. Kratzmann (2020), S. 24. 
Abbildung 12: Hochgerechneter Anteil der leitungsgebundenen Breitbandverfügbarkeit von $50 \mathrm{Mbit} / \mathrm{s}$ der privaten Haushalte und des Gewerbes

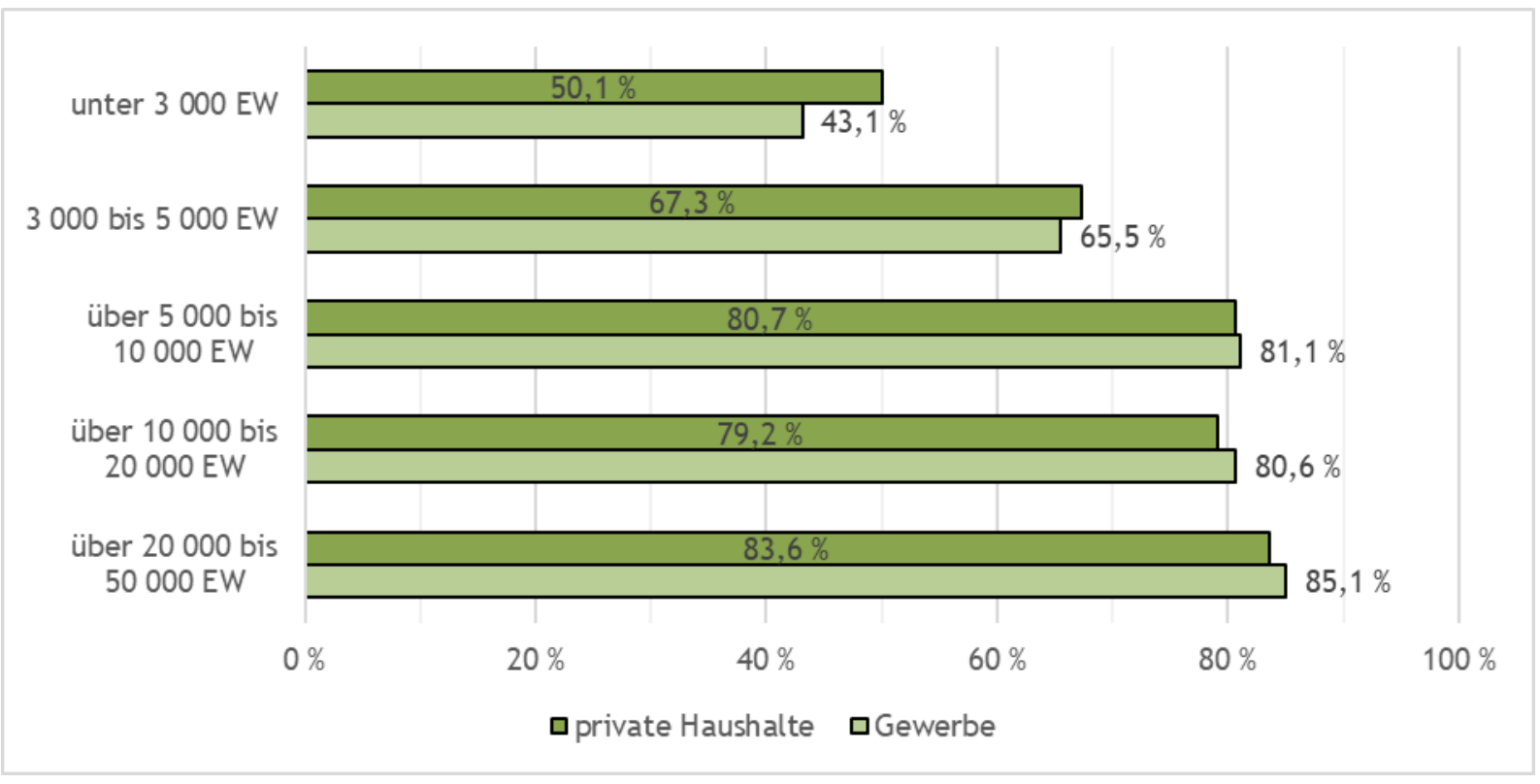

Quelle: Eigene Darstellung; eigene Berechnung, $\mathrm{N}=35$.

Hierbei zeigt sich, dass insbesondere Gewerbe in kleineren Kommunen der EW-Größenklasse von unter $3000 \mathrm{EW}$ nur unzureichend mit Breitband von mindestens $50 \mathrm{M} /$ Bits versorgt sind. Mit zunehmender EW-Größenklasse nimmt dieser Wert jedoch zu. In der EW-Größenklasse von 20000 bis 50000 EW liegt dieser Wert mit 85,1 \% beinahe doppelt so hoch. Die Breitbandverfügbarkeit der privaten Haushalte liegt in den beiden unteren EW-Klassen vor der gewerblichen Nutzung, wobei sich dieser Zustand ab einer EW-Klasse von 5000 bis 10000 EW dreht. Auch wenn die Spannweite im Bereich der privaten Haushalte mit 33, 5 Prozentpunkten weniger stark ausfällt als bei der gewerblichen Nutzung, so ist dieser Wert auch im Bereich der privaten Haushalte als gravierend hoch anzusehen. Diese Beobachtungen decken sich mit jenen aus Kapitel 1, die auf Basis der Daten des Breitbandatlas dargestellt wurden. Damit wird deutlich, dass die kommunale Familie in Sachsen sehr heterogen im Bereich der Breitbandverfügbarkeit aufgestellt ist. Vor dem Hintergrund zunehmender Digitalisierung und der Verbreiterung des Dienstleistungssektors erfolgt somit eine zunehmende Ausdifferenzierung der lokalen wirtschaftlichen Leistungsfähigkeit. Hieraus kann die Problematik „abgehängter Regionen“ entstehen, die auf Grund einer unzureichenden Breitbandversorgung für Unternehmen als unattraktive Unternehmensstandorte wahrgenommen werden und somit im regionalen Standortwettbewerb an Attraktivität verlieren. Dies führt letztlich in eine wirtschaftliche Abwärtsspirale. Neben der Attraktivität als Wirtschaftsstandort und den damit verbundenen arbeitsmarktpolitischen Effekten ergeben sich hieraus weiterführende Implikationen für die privaten Haushalte. Schnelles Internet ist im Zeichen von Corona durch Home-Office- und Home-Schooling-Nutzung ebenfalls als wichtige Rahmenbedingung anzu- 
sehen. ${ }^{22}$ Dabei muss betont werden, dass sich beide Themenbereiche gegenseitig beeinflussen: Durch eine gewerbliche Breitbandversorgung kann eine wirtschaftliche Grundlage für neue Geschäftszweige entstehen, wodurch zusätzliche Arbeitsplätze entstehen können. Gleichzeitig kann eine flächendeckende Breitbandversorgung auch als Standortfaktor für das Wohnen angesehen werden, der es privaten Haushalten abseits der wirtschaftlichen Zentren ermöglicht, sich niederzulassen.

Um diese Gemengelage vertieft zu analysieren und mögliche Effekte differenziert darstellen zu können, wird hier eine Unterscheidung zwischen Tiefbaumaßnahmen auf der einen und dem eigentlichen Breitbandausbau auf der anderen Seite getroffen, in Kenntnis, dass beide Themenbereiche Überschneidungen aufweisen. Dahingehend wird im darauffolgenden Kapitel 3 die Verknüpfung der einzelnen Ergebnisse aus den beiden Teilbereichen in den Fokus der Betrachtung gestellt.

\subsubsection{Teil B: Tiefbaukoordinierung}

Im Rahmen der Befragung sind Meinungen und Positionen zur Tiefbaukoordinierung abgefragt worden. Hierbei zeigt Abbildung 13, ob Maßnahmen im Tiefbau vermehrt gebündelt oder immer separat durchgeführt worden sind. ${ }^{23}$

22 Vgl. Rottmann et al. (2020).

23 In Abbildung 13 werden die Ergebnisse in ungewichteter Form, einwohnergewichteter Darstellung und in einer Gewichtung nach einem Zersiedlungsindex widergegeben. Um eine Übersichtlichkeit zu gewährleisten, wird anschließend nur auf die einwohnergewichteten Ergebnisse eingegangen. Sofern jedoch nennenswerte Abweichungen zu der Gewichtung nach dem Zersiedlungsindex oder der ungewichteten Variante vorliegt, werden die Ergebnisse ebenfalls im Text beschrieben. 
Abbildung 13: Führen Sie verschiedene Baumaßnahmen im Tiefbau innerhalb Ihrer Kommune oder im Rahmen einer interkommunalen Kooperation gebündelt durch?

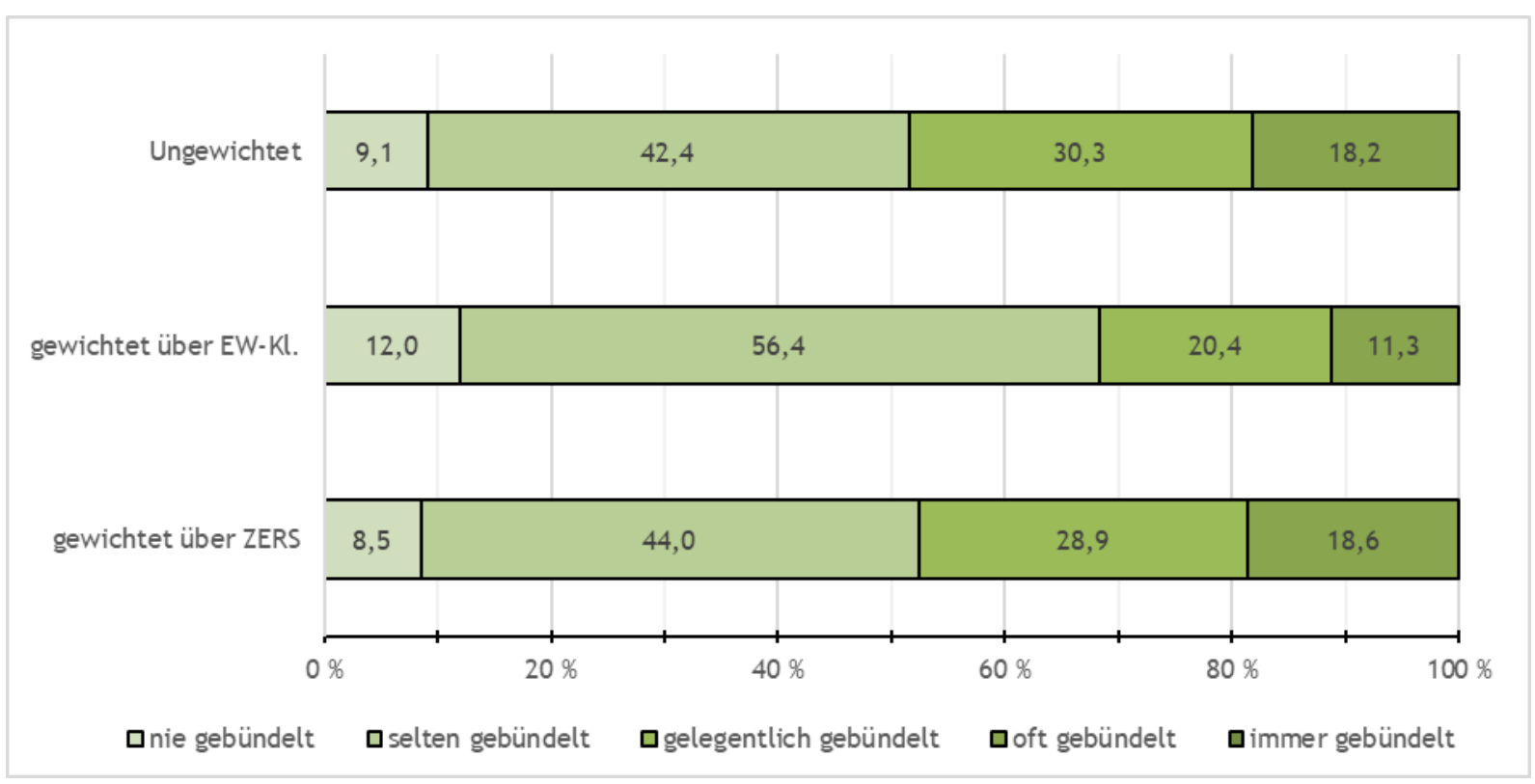

Quelle: Eigene Darstellung; eigene Berechnung, $N=33$.

Vor dem Hintergrund einer zunehmenden Belastung der öffentlichen Verwaltung kann die Bündelung von Baumaßnahmen ebenfalls ein Mittel zur Abhilfe darstellen. Hierbei zeigt sich, dass bei allen Gewichtungen in keinem Fall eine Bündelung für alle Tiefbaumaßnahmen erfolgt. Damit kann geschlussfolgert werden, dass eine Bündelung von Tiefbaumaßnahmen nicht immer vorteilhaft ist. Rund 12 \%der Kommunen bündeln niemals ihre Tiefbaumaßnahmen, während der Großteil mit rund 56 \% eine Bündelung selten vornimmt. Oft, beziehungsweise gelegentlich, bündeln derweil rund 31,7 \%aller Kommunen ihre Tiefbaumaßnahmen. Wird der Zersiedlungsindikator ${ }^{24}$ als Gewichtungsparameter herangezogen, so zeigt sich, dass sich die Verteilung der Antwortmöglichkeiten sich nicht sonderlich von der ungewichteten Variante unterscheidet. Rund 47,5\% aller Kommunen fassen demnach ihre Tiefbaumaßnahmen zusammen. Demnach nimmt nicht nur der Anteil derer ab, die nie ihre Tiefbaumaßnahmen bündeln, sondern auch der Anteil derer, die nur selten eine Bündelung vornehmen. Die Verschiebung kann indes auf die Wahl des Gewichtungsindikators zurückgeführt werden. Dabei ist eine Landschaft umso stärker zersiedelt, je mehr Fläche bebaut und diese gestreut ist und je geringer die Ausnutzung für Wohn- und Arbeitszwecke ist. ${ }^{25}$ Hingegen scheinen die Breitbandverfügbarkeit von über 50 Mbit/s oder die VZÄ im Bauamt keine sig-

24 Für eine extensive Beschreibung des Zersiedlungsindexes siehe Anhang: Gewichtung und Hochrechnung oder alternativ IÖR-Monitor (2020).

25 Eine genaue Darstellung der Methodik siehe J eager et al. (2018). Die Daten wurden vom Institut für ökologische Raumordnung im Rahmen des IÖR-Monitors bereitgestellt. 
nifikante Erklärung auf das Antwortverhalten im Sinne eines Gewichtungsparameters zu geben. Eine weitere Differenzierung nach Einwohnerklassen zeigt - und bestätigt die Befunde -, dass insbesondere Kommunen unter 5000 Einwohnern eher bündeln ${ }^{26}$.

Dies zeigt, dass im Bereich der Bündelung von Tiefbaumaßnahmen weiterhin Potenzial besteht. Kommunen, die eine tendenziell zersiedelte Fläche aufweisen, neigen eher dazu, ihre Tiefbaukapazitäten zusammenzufassen. Eine Bündelung ist vor dem Hintergrund von zerstreuten bebauten Flächen als durchaus vorteilhaft zu interpretieren, da durch die vergleichsweise unkonzentrierte Fläche zusätzliche Kosten in der Bewirtschaftung entstehen können. Extern können durch die Bündelung von Maßnahmen somit beispielsweise Kosteneinsparungen in der Ausschreibung durch bessere Angebotspreise erzielt werden. Hingegen können intern Transaktionskosten vermieden werden, da Zuständigkeiten und Verantwortlichkeiten innerhalb der Verwaltungen ebenfalls konzentriert werden können.

Dahingehend zeigt Abbildung 14 die Hindernisse auf, die eine weiterführende Koordinierung von Tiefbaumaßnahmen behindern. Dabei zeigt sich, dass rechtliche Auflagen, Widerstände übergeordneter öffentlicher Ebenen, Vereinbarungskosten und lokale Egoismen keine nennenswerten Hindernisse im Rahmen der Koordinierung von Tiefbaumaßnahmen aus Sicht der befragten Kommunen darstellen. Hingegen bestätigt sich mit Bezug auf die unzureichende Personalausstattung der Befund, der bereits durch Abbildung 11 angerissen worden ist. Rund 55 \%aller Befragten gaben an, dass die Personalausstattung ein Problem bei der Koordinierung von Tiefbaumaßnahmen darstellt. Dabei hat sich dieser Befund durch Einwohnergewichtung selbst nochmals verstärkt, was den Rückschluss zulässt, dass eher kleine Kommunen unter $3000 \mathrm{EW}$ durch eine unzureichende Personalstruktur gekennzeichnet sind. ${ }^{27}$ Zusätzlich unterstreicht eine moderat negative Korrelation einen mittleren Effekt zwischen $50 \mathrm{Mbit} / \mathrm{s}$ $(\mathrm{HH})$ und dem Antwortverhalten der befragten Kommunen in Bezug auf die Personalausstattung $^{28}$ und unklare Zuständigkeit ${ }^{29}$. Umso höher demnach die Versorgung mit 50 Mbit/s der Haushalte ist, desto geringer sind die Problematiken, welche mit einer ungenügenden Personalausstattung und unklaren Zuständigkeiten einhergehen. Ebenso besteht eine mittlere negative Korrelation zwischen der Personalausstattung und der Anzahl an VZÄ: Kommunen mit vergleichsweise weniger Personal stimmen auch eher dem Hindernis „unzureichende Personalausstattung“ zu. Dies trifft auch auf das Hindernis „unklare Zuständigkeiten“ zu $u^{30}$. Dies zeigt, dass eine einfache Skalierbarkeit von öffentlicher Verwaltung nicht gegeben ist.

26 Signifikanzniveau $5 \%$ \% . Hinweis: Im Weiteren wird aufgrund des Vorliegens von ordinalen Daten die Rangkorrelation gebildet, um etwaige Zusammenhänge zu prüfen.

27 Wie aus Abbildung 7 ersichtlich, ist die EW Gruppe unter 3000 in der Stichprobe unterrepräsentiert, was durch die Einwohnergewichtung ausgeglichen wird.

28 Signifikanzniveau: $15 \%$.

29 Signifikanzniveau: $5 \%$ *

30 Signifikanzniveau: $5 \%$. 
Es bedarf demnach nicht nur mehr Personal, sondern auch einer übersichtlichen Verwaltungsstruktur. Zusätzlich weisen Kommunen unter 5000 eine höhere Zustimmung zu den in Abbildung 14 dargestellten Hindernissen auf.

In Bezug auf unklare Zuständigkeiten übergeordneter Einheiten ist der Zustimmungsgrad insgesamt relativ hoch, wie durch die drei großen Blöcke von "neutral“ bis "stimme voll zu“ ersichtlich wird. Diesem Hindernis stimmen bei einer gewichteten Betrachtung insgesamt rund 53 \%aller Kommunen zu. Auch hier liegt eine Verzerrung aufgrund der Einwohnergröße vor, die mithilfe der Gewichtung partiell ausgeglichen werden konnte. Somit scheinen kleinere Kommunen neben einer unzureichenden Personalausstattung auch unter unklaren Zuständigkeitsverhältnissen bei übergeordneten Ebenen zu leiden. Hierbei stehen beide Erkenntnisse miteinander im Verhältnis, da durch einen geringen Bestand an VZÄ ein größerer Aufgabenbereich durch weniger Personal abgebildet werden muss.

Abbildung 14: Stimmen Sie aus Sicht Ihrer Kommune zu, dass die folgenden Hindernisse im Rahmen der Koordinierung von Tiefbaumaßnahmen bestehen?

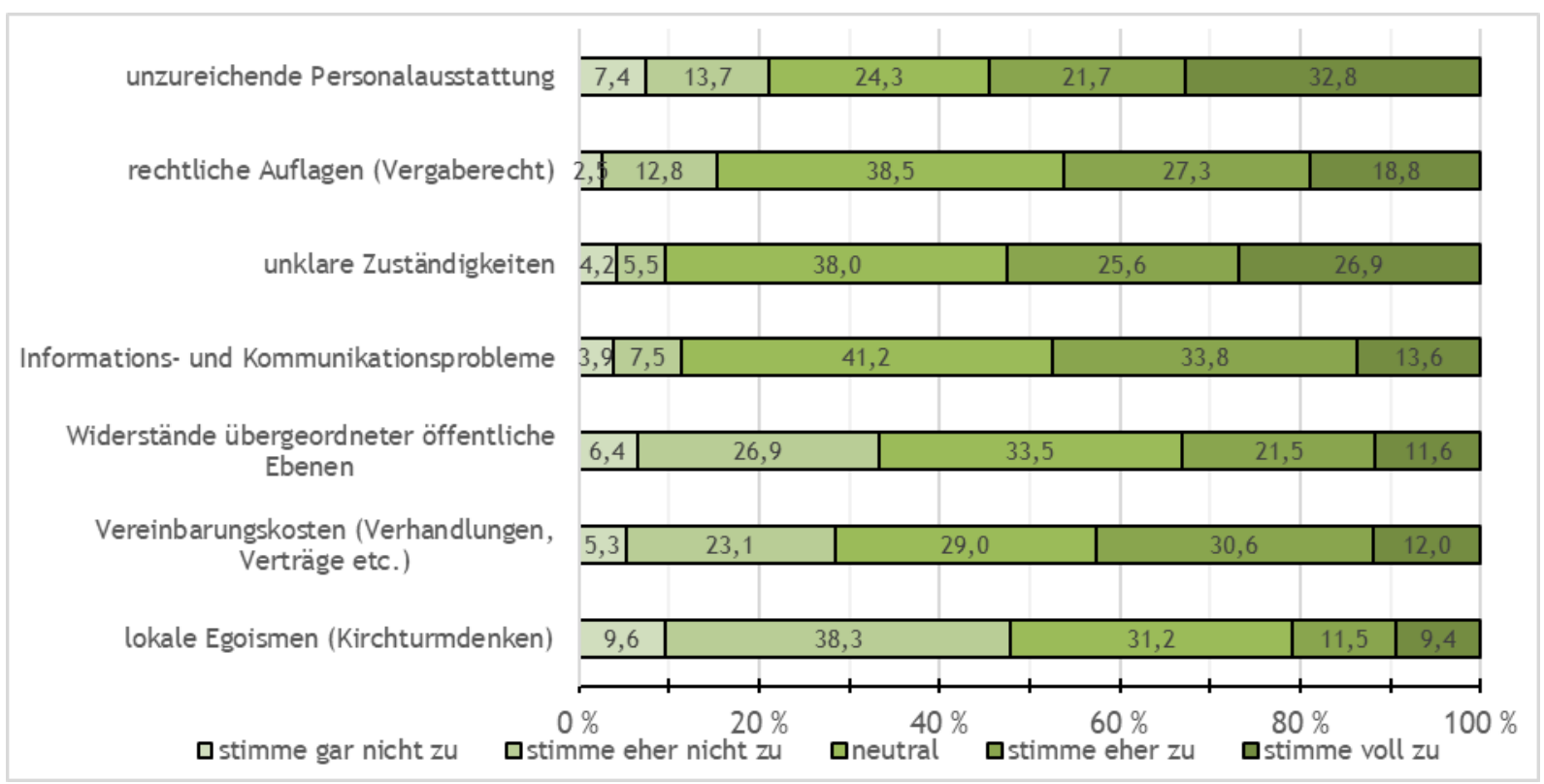

Quelle: Eigene Darstellung; eigene Berechnung, $\mathrm{N}=33$.

Dagegen nimmt der Anteil der Kommunen, die dem Hindernis der Informations- und Kommunikationsprobleme neutral gegenüberstehen in einer Einwohnergewichtung zu. Dies zeigt, dass eher größere Kommunen durch Informations- und Kommunikationsprobleme, folglich auch Transaktionskosten, geprägt sind. Dies ist vor dem Hintergrund einer tendenziell ausdifferenzierteren kommunalen Verwaltung jedoch nicht verwunderlich. Während kleinere Kommunen eher durch externe Informationsasymmetrien geprägt sind, liegen demnach in größeren kommunalen Einheiten vermehrt interne Informationsungleichheiten vor, die dazu führen, dass Koordinierungsbemühungen im Rahmen von Tiefbaumaßnahmen nicht vollständig erfolgreich verlaufen. 
Um die vorliegenden Hemmnisse zu minimieren, stellt sich die Frage, welche Instrumente zur Förderung der Koordinierungsbemühungen als effektiv eingeschätzt werden. Hierzu gibt Abbildung 15 Auskunft. Die Antwortmöglichkeiten "Leitfaden“ und „Weiterbildungsmöglichkeiten" sind als nicht sonderlich effektiv bewertet worden.

Abbildung 15: Wie schätzen Sie die Instrumente zur Förderung der Koordinierung im kommunalen Tiefbau hinsichtlich ihrer Effektivität ein?

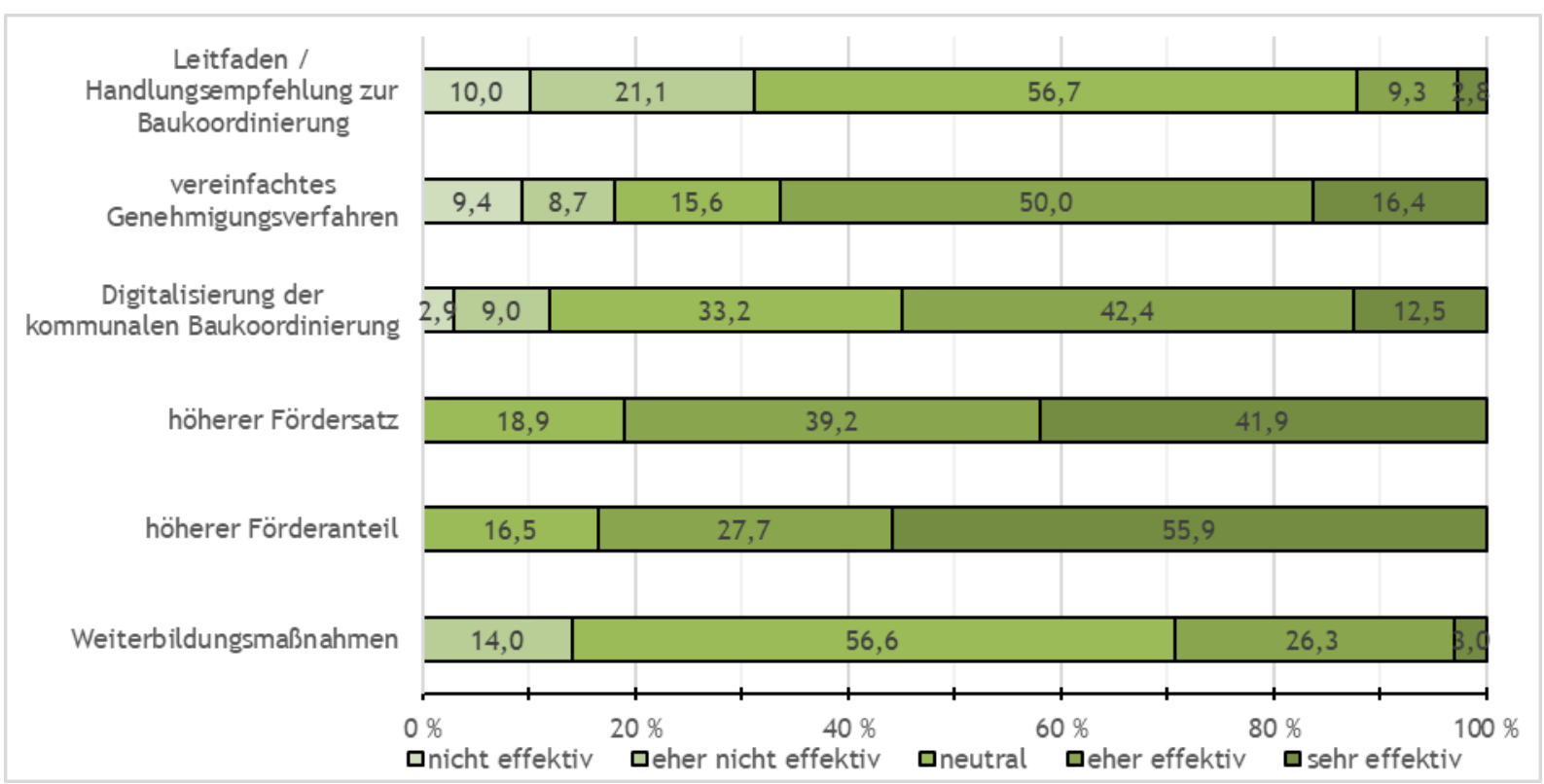

Quelle: Eigene Darstellung; eigene Berechnung; $N=31$.

Das vereinfachte Genehmigungsverfahren stieß dabei auf sehr hohe Zustimmung. Der gewichtungsbedingte Unterschied liegt hier bei 6, 3 Prozentpunkten und insbesondere Kommunen mit weniger als $10000 \mathrm{EW}$ stimmten dieser Aussage zu. Dennoch scheint über die Einwohnerklassen hinweg ein vereinfachtes Genehmigungsverfahren ein probates Lösungsmittel für eine effizientere Koordinierung des kommunalen Tiefbaus zu sein. Die Digitalisierung der kommunalen Baukoordinierung wird gewichtet von rund 55 \%als erstrebenswert angesehen. Ungewichtet liegt diese Einschätzung knapp neun Prozentpunkte niedriger. Folglich sehen kleinere Kommunen, insbesondere unter 10000 EW, weiteren Bedarf in der Digitalisierung der Baukoordinierung, während größere Kommunen diesen Bedarf als weniger wichtig einschätzen. Dies hängt unmittelbar mit dem Stand der Digitalisierung und somit auch dem Stand der Umsetzung des Onlinezugangsgesetzes (OZG) zusammen. Dies kann durch eine ausdifferenzierte und spezialisierte Verwaltung tendenziell effektiver umgesetzt werden, wodurch tendenziell kleinere kommunale Verwaltungen ins Hintertreffen geraten. Zudem stimmen in der Tendenz Kommunen mit einer geringeren Breitbandverfügbarkeit dieser Aussage zu. Eine statistisch signifikante Abweichung im Antwortverhalten konnte für Kommunen mit einer geringen Anzahl an VZÄ-Stellen im Bauamt nachgewiesen werden, diese stimmten 
der Aussage überproportional zu. ${ }^{31}$ Dies erscheint nachvollziehbar, da Kommunen mit weniger entsprechenden Kapazitäten, durch komplexe Genehmigungsverfahren zusätzlich gefordert werden.

Als effektiv werden durchweg die Möglichkeiten höherer Fördersätze und höherer Förderanteile gesehen. ${ }^{32}$ Gerade Kommunen unter $5000 \mathrm{EW}$ stimmen beiden Aussagen überproportional deutlich zu. 81 \%halten einen höheren Fördersatz als probates Mittel zur Förderung der Koordinierung der kommunalen Tiefbauaktivitäten. ${ }^{33}$ Neben den allgemein höheren Fördersätzen steht ebenfalls die Möglichkeit im Raum, die Förderanteile zu erhöhen. Für die Fördersätze hat der Großteil der Befragten mit rund 84 \% angegeben, dass verminderte Eigenanteile für Fördermittel eine Lösungsoption darstellen würde. Insbesondere Kommunen mit einer geringen Personalausstattung in den Bauämtern sehen einen höheren Förderanteil als zielführend $\mathrm{an}^{34}$. Da diese kleineren Kommunen angehören, könnte dieser Zusammenhang daraufhin deuten, dass es für diese in Sachsen besonders aufwendig ist, die geforderten Eigenanteile für Förderprogramme aufzubringen. Beide Optionen, der erhöhte Fördersatz, wie auch der höhere Förderanteil, laufen letztlich auf eine verstärkte finanzielle Förderung für die kommunale Ebene hinaus. Beide Antworten sind jedoch nicht miteinander gleichzusetzen. Die Nutzung von Fördermitteln wird erheblich durch die eigene kommunale Leistungsfähigkeit determiniert. Dies liegt darin begründet, dass Eigenmittel aufgebracht werden müssen, um Fördermittel erhalten zu können. Dahingehend wird durch den vorzuweisenden Eigenanteil in die Verteilung der Fördermittel eingegriffen. Durch die Reduktion des benötigten Eigenanteils stehen Fördermittel somit gerade jenen Kommunen offen, die nur über ein geringes Volumen an Eigenfinanzierungsmöglichkeiten verfügen. Dagegen zielt die Erhöhung des Fördersatzes via FAG auf die Bedarfe ab, die augenscheinlich vorliegen. Hier ist jedoch interpretatorische Vorsicht geboten. Fördermittelrichtlinien fokussieren konkrete Fördergegenstände. Die korrespondierenden Mittel haben demnach einen Verwendungszweck, der durch Nachweispflichten von Seiten der Kommune im gesamten Bau- und Instandhaltungsprozess zu erbringen sind. Dahingehend scheint ein derart hoher Anteil an Befragten, die einen erhöhten Förderbedarf sehen, nicht mit dem Diktum „einfach mehr Geld“

31 Signifikanzniveau $5 \%$ \%

32 Im allgemeinen Sprachgebrauch werden die Termini Förderanteil und Fördersatz als Synonyme verwendet. In den nachfolgenden Ausführungen beziehen sich die Förderanteile auf die prozentuale Förderung durch die übergeordneten staatlichen Ebenen im Rahmen der Fördermittelprogramme. Fördersätze beziehen sich indes auf die Förderung durch das FAG, welche beispielsweise pauschaliert erfolgen kann.

33 Bereits in der Studie von Bender/ Mengs (2020): Mobilität in Sachsen konnte herausgestellt werden, dass die Investitions- und Instandhaltungsbedarfe oftmals über den eigentlichen Fördermittelvolumina liegen. Siehe hierzu: Bender/ Mengs (2020), S. $19 \mathrm{ff}$.

34 Signifikanzniveau $5 \%$. 
gleichzusetzen zu sein. Vielmehr deutet der hohe Anteil derer, die fiskalische Nachholbedarfe sehen, darauf hin, struktureller Natur zu sein. ${ }^{35}$

In den beiden folgenden Abbildungen wird die Dokumentation vorhandener Leitungen thematisiert. Dieser Aspekt wurde im Vorhinein als möglicher kritischer Faktor für eine erfolgreiche Koordinierung im Tiefbau identifiziert. Zudem sind die Art der gespeicherten Information und der Zugang zu dieser in den Kommunen unterschiedlich geregelt.

Abbildung 16: Gibt es eine Dokumentation über vorhandene Leitungen oder anderer Infrastrukturen in Ihrer Kommune?

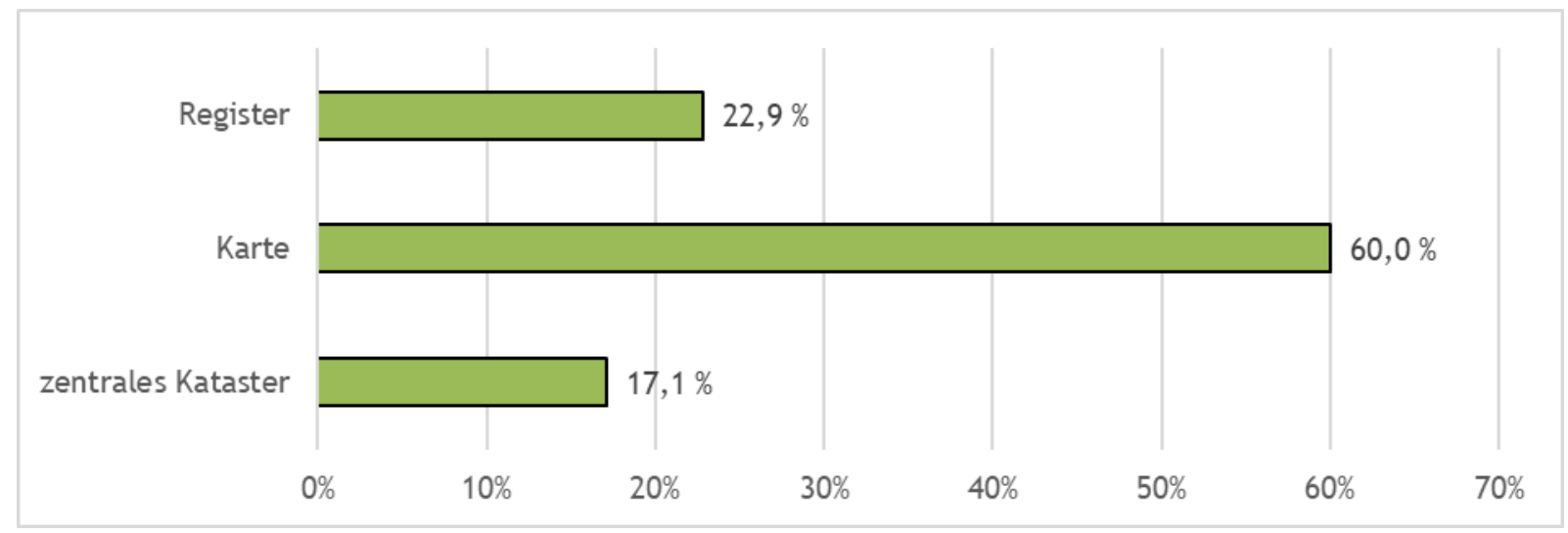

Quelle: Eigene Darstellung; eigene Berechnung, $\mathrm{N}=35$.

Auf die Frage hin, ob eine Dokumentation vorhandener Leitungen oder anderer Infrastrukturen in der jeweiligen Kommune vorliegen, ist mehrheitlich, wie Abbildung 16 zeigt, die Dokumentation als Karte gewählt worden. Ein zentrales Kataster und ein Register liegen in etwa gleichauf. Damit sind georeferenzierte Daten in Kartendarstellungen derzeit mit $60 \%$ der Angaben das meistgenutzte Medium der Befragten zur Dokumentation.

Fraglich ist jedoch, wie der Zugang zu diesen Daten organisiert ist. Hierzu gibt Abbildung 17 einen Überblick.

35 Zu dem Thema der Instandhaltungs- und Investitionsbedarfe, siehe Sydow/ Hesse (2020). 
Abbildung 17: Wie ist der Zugang zu den Informationen für Dritte zurzeit in Ihrer Kommune gestaltet?

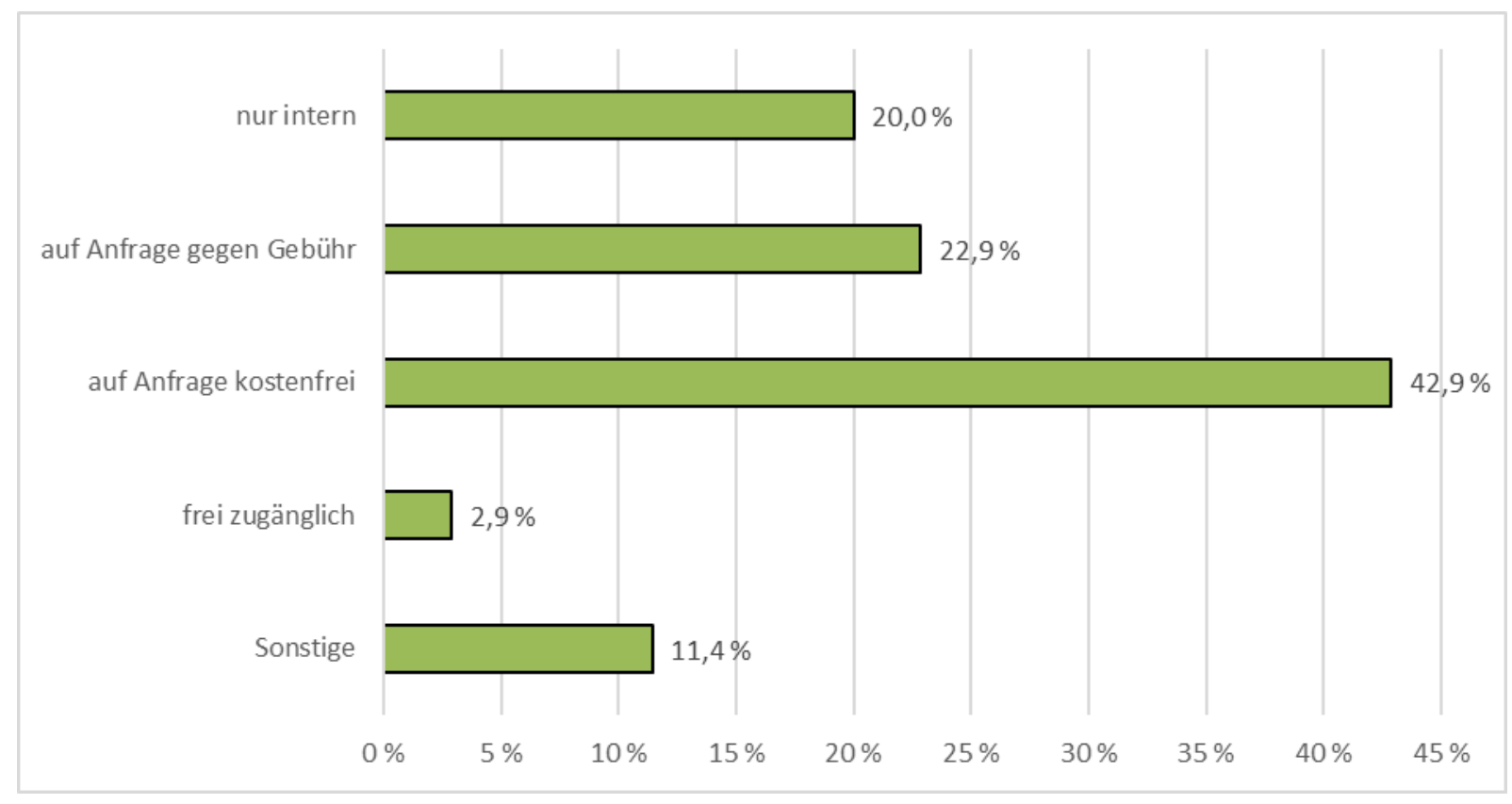

Quelle: Eigene Darstellung; eigene Berechnung; $\mathrm{N}=35$.

Die meisten Befragten geben an, auf Anfrage kostenfreie Daten zur Verfügung zu stellen. Mit Abstand folgt die Anfrage gegen eine Gebühr. Zudem haben 20 \%angegeben, die Daten über vorhandene Leitungen nur für den internen Gebrauch zu nutzen. Somit wird der Großteil der vorhandenen Daten zwar zur Verfügung gestellt, es findet jedoch eine Bedarfsprüfung statt. Somit muss durch den Dritten dargelegt werden, dass dieser ein berechtigtes Interesse daran hat, die angeforderten Daten abzurufen. Dies ist nicht nur aus Gründen des Datenschutzes von Relevanz, sondern auch, da es sich um kritische Infrastruktur handelt.

Aus der Verknüpfung beider Fragen zeigt sich, dass die meisten befragten Kommunen ein kartenbasiertes System zur Speicherung der Leitungsdaten und anderer Infrastrukturen nutzen, das jedoch auf Anfrage unter weiterführenden Bedingungen kostenfrei zur Verfügung gestellt wird.

Indes stellt sich die Frage, wie die Bedeutung folgender Daten zur Speicherung eingeschätzt wird und ob diese die beschriebenen Koordinierungshindernisse reduzieren können. 
Abbildung 18: Wie schätzen Sie die Bedeutung der Speicherung folgender Informationen hinsichtlich ihrer Wichtigkeit für eine erfolgreiche Koordinierung von kommunalen Tiefbaumaßnahmen ein?

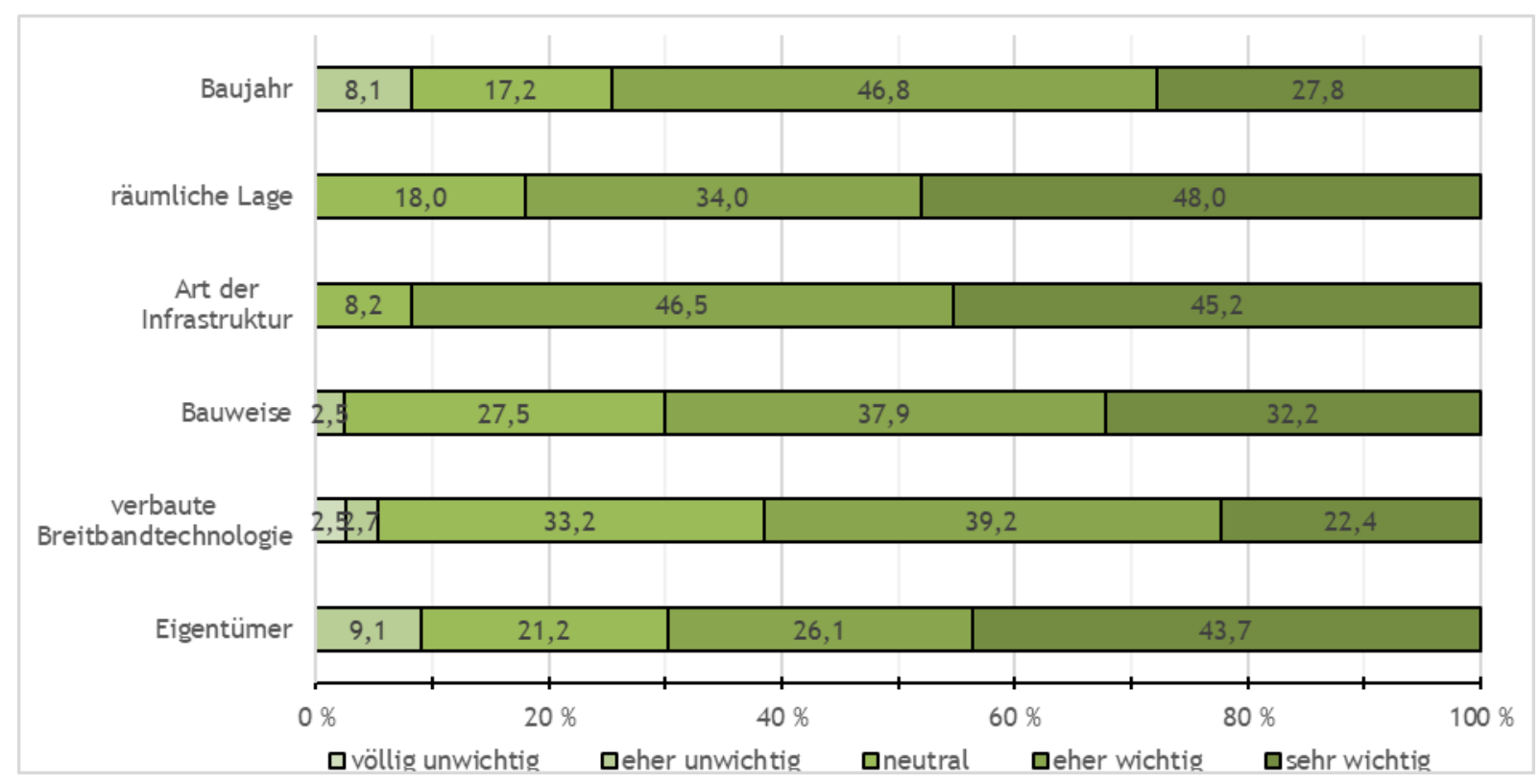

Quelle: Eigene Darstellung; eigene Berechnung, $\mathrm{N}=30$.

Abbildung 18 stellt die Antwortoptionen in einwohnergewichteter Variante dar. Hierbei zeigt sich, dass die Kommunen die verbaute Breitbandtechnologie als vergleichsweise unwichtig ansehen. Allerdings schätzen Kommunen unter 5000 EW sowie Kommunen mit einer geringen Breitbandverfügbarkeit von 50 Mbit/s diesen Aspekt als wichtiger ein. Als sehr wichtig werden hingegen mehrheitlich die räumliche Lage, die Art der Infrastruktur und die Eigentumsverhältnisse gesehen. Gerade Bauämter mit geringen Personalausstattung sehen in der Tendenz einen höheren Mehrwert darin, über Geodaten zu verfügen. Die Bedeutung der Bauweise, wie auch des Baujahrs, nimmt in der einwohnergewichteten Variante zu. Dies lässt darauf schließen, dass gerade für kleinere Kommunen diese beiden Informationen als zentral erachtet werden können. Zudem empfinden Kommunen mit weniger VZÄ in den Bauämtern diese Informationen als wichtiger. Kommunen die bezogen auf ihre Gesamtfläche wenig Einwohner aufweisen, schätzen dies ebenfalls als wichtig ein. ${ }^{36}$ Diese zuletzt genannten Korrelationen unterstützen die These, dass kleinere Bauämter und sehr ländlich geprägte Kommunen tiefergehende Daten in einer Infrastrukturdokumentation schätzen.

36 Signifikanzniveau: $10 \%$. 


\subsubsection{Teil C: Breitband}

Im dritten Teil der Befragung wurden spezifische Aspekte zur Umsetzung des öffentlich geförderten Breitbandausbaus untersucht. Dabei wurde zuerst nach den Treibern auf der Nachfrage- und Angebotsseite für den Iokalen Breitbandausbau gefragt, wie aus Abbildung 19 ersichtlich wird.

Abbildung 19: Wie bewerten Sie die nachfolgenden Faktoren für den lokalen Breitbandausbau bezogen auf Ihre Kommune?

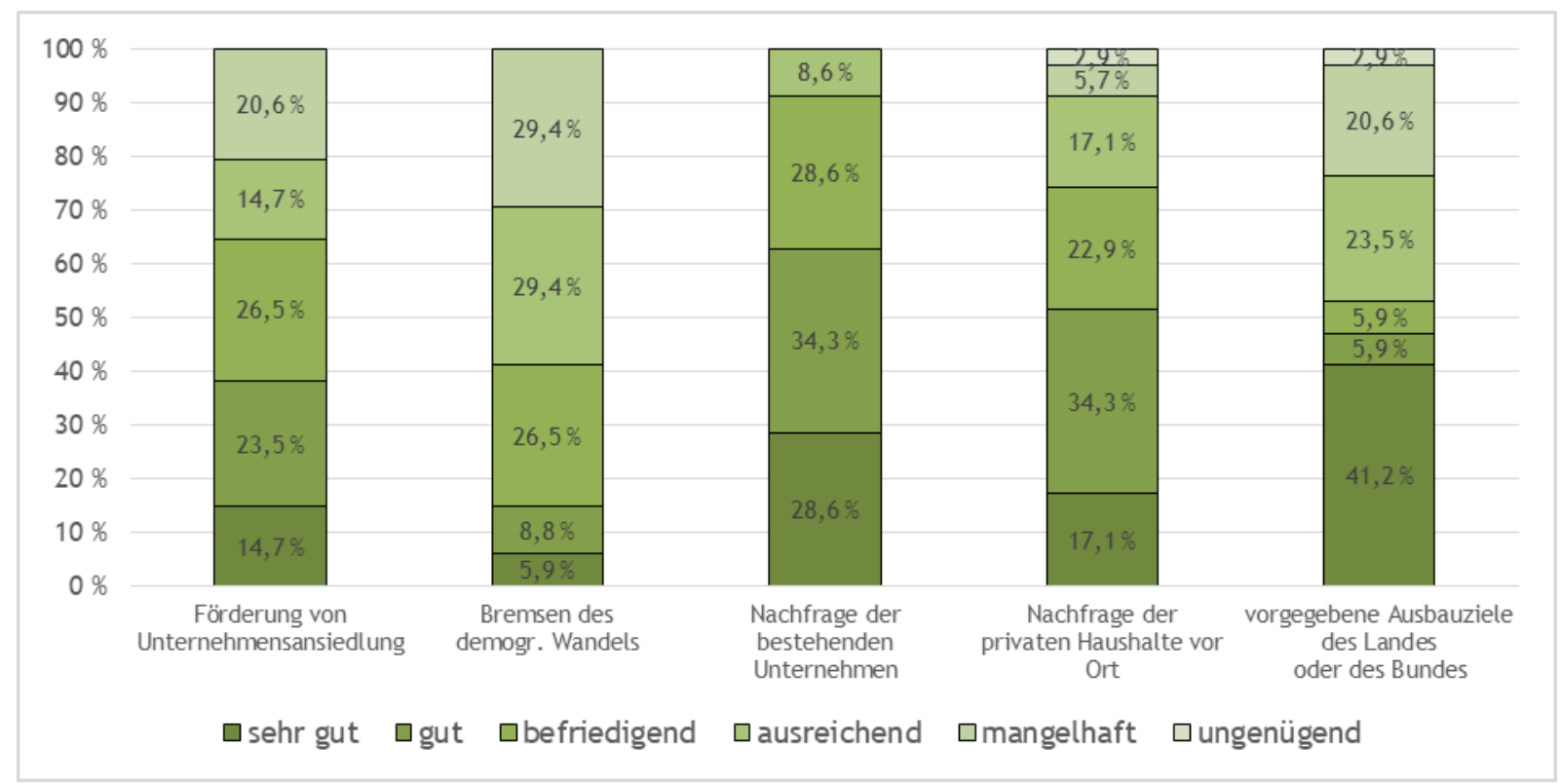

Quelle: Eigene Darstellung; eigene Berechnung, $N=35$.

Dabei zeigt sich, dass insbesondere die vorgegebenen Ausbauziele des Landes und des Bundes von gesonderter Relevanz sind. Die Nachfrage der bestehenden Unternehmen ist an zweiter Stelle zu nennen, gefolgt von der Förderung der Unternehmensansiedlung. Als "gut" wird die Nachfrage der privaten Haushalte vor Ort und die Nachfrage der bestehenden Unternehmen bewertet. Den höchsten negativen Wert hat der Faktor demografischer Wandel. Hierdurch bestätigt sich die These, dass die Erfolgsfaktoren für die Umsetzung des lokalen Breitbandausbaus eher in angebotsseitigen und unternehmensbezogenen Effekten zu finden sind, gefolgt von nachfrageorientierten Einflüssen der privaten Haushalte, unabhängig der Altersstruktur. ${ }^{37}$

Um dieses Bild zu verifizieren, wurde in der nachfolgenden Frage nach den Bereichen gefragt, für die die Bedeutung des Breitbandausbaus als besonders hoch eingeschätzt wird, wie Abbildung 20 zeigt. Hierbei wurden die Gewerbe und Dienstleistungen mit 35, 3 \%am höchsten gewertet, gefolgt von den Privathaushalten. Mit großem Abstand folgen der Bildungssektor und die Digitalisierung der Verwaltung. Somit zeigt sich einerseits, dass sich das oben

37 Vgl. Mengs/ Günther (2018). 
gezeichnete Bild mit den Angaben der zweiten Frage deckt. Andererseits wird jedoch deutlich, dass die Bedeutung der Digitalisierung der Verwaltung, welche insbesondere durch das OZG an Gewicht zunehmen wird, bisher als eher gering eingeschätzt wird. Dies kann entweder darin begründet sein, dass die kommunale Verwaltung bereits größtenteils digitalisiert ist, oder dass die Herausforderung der Umsetzung des OZG noch nicht als solche wahrgenommen wird. Es könnte ebenfalls darin begründet sein, dass der Breitbandausbau in den entsprechenden Kommunen bereits weiter fortgeschritten ist und nicht als weiter relevant für die fortschreitende Digitalisierung der Verwaltung gesehen wird.

Abbildung 20: Für welche Bereiche schätzen Sie die Bedeutung des Breitbandausbaus aus Sicht Ihrer Kommune als besonders hoch ein?

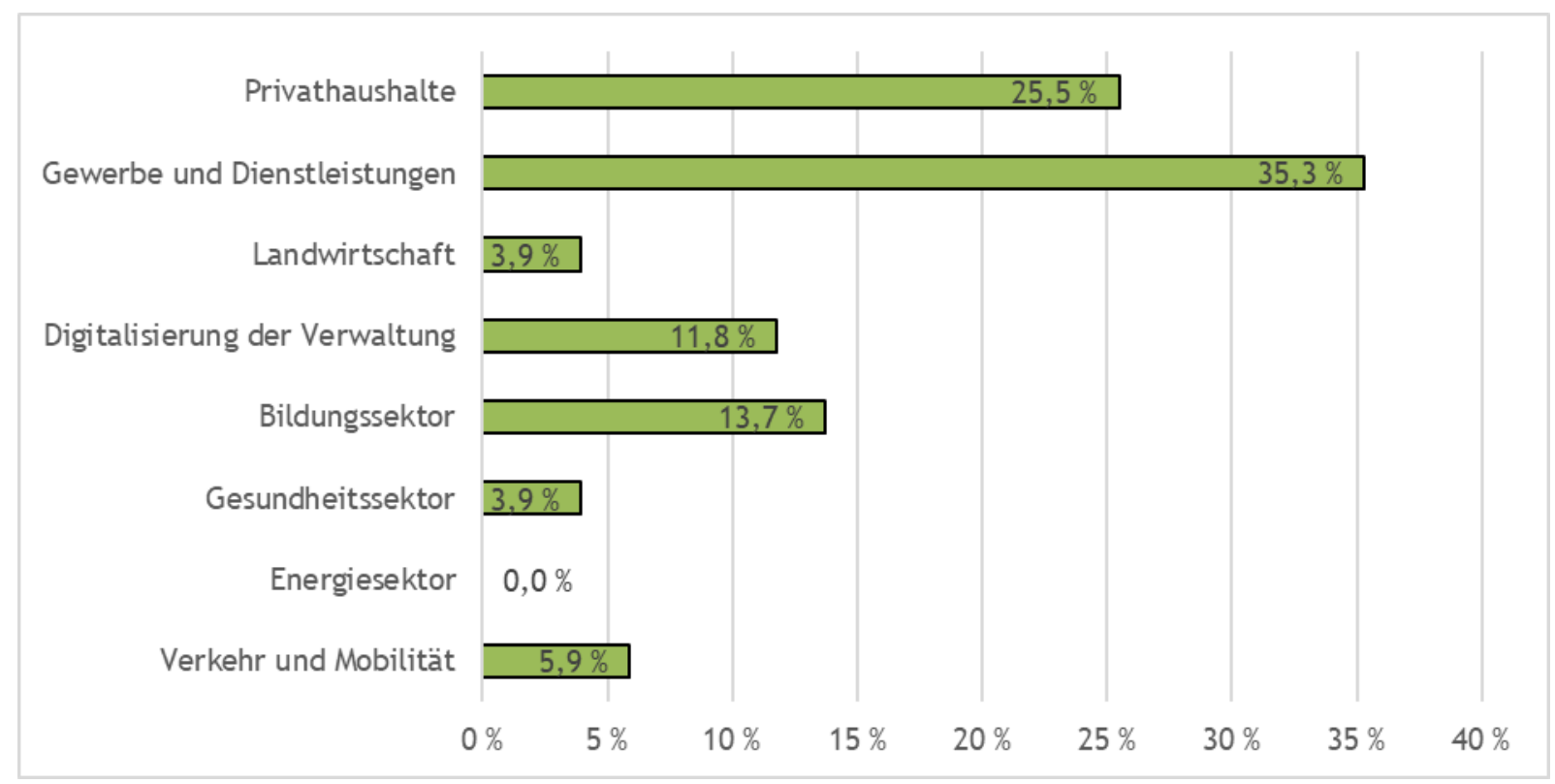

Quelle: Eigene Darstellung; eigene Berechnung, $N=36$.

Auch der Gesundheitssektor sowie der Bereich Verkehr und Mobilität wird als eher nachrangig im direkten Vergleich zum Unternehmens- und Haushaltssektor angesehen. Dies unterstreicht nochmals die Bedeutung der beiden letztgenannten und zeigt auf, dass auch im Rahmen des Standortwettbewerbs der Breitbandausbau von entscheidender Bedeutung ist, um private Haushalte zu binden und Unternehmensansiedlungen zu befördern. 
Abbildung 21: Haben Sie als Kommune oder eines Ihrer kommunalen Unternehmen bereits ein oder mehrere Proj ekte zum Breitbandausbau durchgeführt oder begleitet?

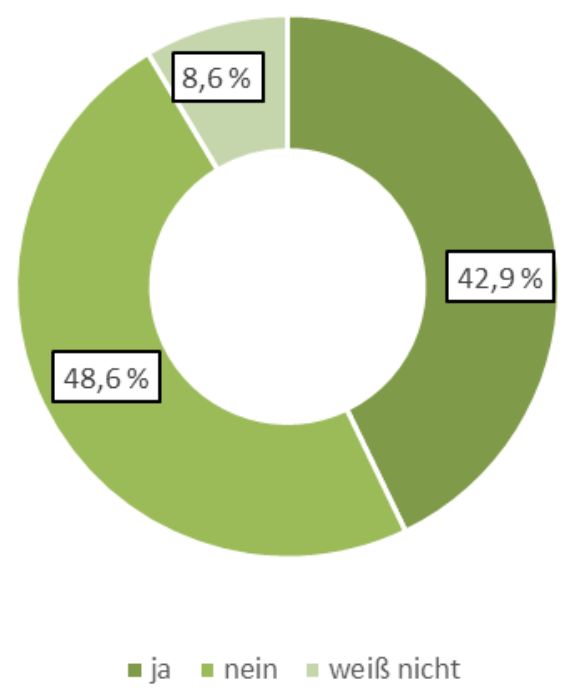

Quelle: Eigene Darstellung; eigene Berechnung, $N=35$.

Weiterhin wurde erfragt, ob bereits Projekte zum Thema Breitbandausbau in der Kommune durchgeführt worden sind, wie Abbildung 21 zeigt. Hierbei wird deutlich, dass die Mehrzahl an Teilnehmern (rund $49 \%$ ) bereits entweder Projekte zum Breitbandausbau durchgeführt (Betreibermodell) oder begleitet hat (Wirtschaftlichkeitslückenmodell). Rund 43 \%gaben dagegen an, bisher kein Projekt zum Breitbandausbau getätigt oder begleitet zu haben. Somit kann die Projekterfahrung in Bezug auf den Breitbandausbau als gespalten angesehen werden. $^{38}$

Eine räumlich ungleich verteilte Breitbandverfügbarkeit trägt unmittelbar zur Heterogenität der kommunalen wirtschaftlichen Landschaft bei, da die Breitbandverfügbarkeit als Standortfaktor angesehen werden kann und somit ein Einflussfaktor in der Determinierung zukünftiger wirtschaftlicher Leistungsfähigkeit darstellt. Vor dem Hintergrund der zunehmenden Digitalisierung und der sich ausdifferenzierenden Breitbandverfügbarkeit können sich somit die wirtschaftlichen Rahmenbedingungen zunehmend ausdifferenzieren. Aus diesem Beweggrund wurde folglich, wie Abbildung 22 zeigt, nach den Hemmnissen für den öffentlich geförderten Breitbandausbau gefragt um zu beschreiben, wodurch sich die beschriebene nachteilige Wettbewerbs- und Standortposition ergibt.

38 Zudem besteht auch die Möglichkeit, dass eventuell ein Projekt nicht erforderlich war, da die Privatwirtschaft den Ausbau betrieben hat. Die Frage zielt jedoch auf die kommunale Expertise und den Wissenstransfer $a b$, der durch die kommunale Beteiligung im Zuge des Breitbandausbaus angezeigt wird. 
Abbildung 22: Für wie wichtig schätzen Sie aus Sicht Ihrer Kommune die folgenden Hemmnisse des öffentlich geförderten Breitbandausbaus ein?

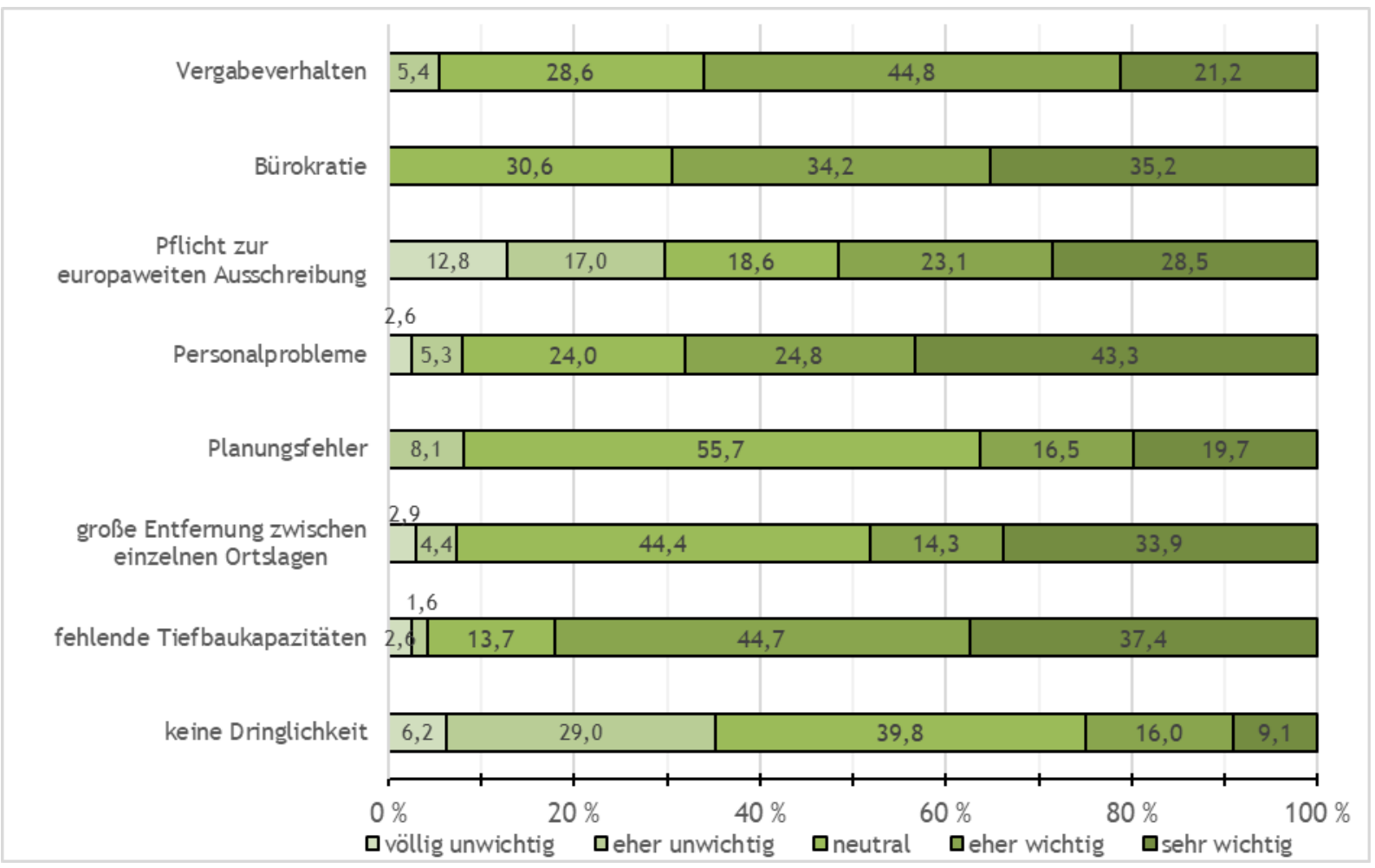

Quelle: Eigene Darstellung; eigene Berechnung, $\mathrm{N}=29$.

Dabei zeigt sich, dass insbesondere die große Entfernung zwischen einzelnen Ortslagen als problematisch angesehen werden kann. Wird hierbei eine Einwohnergewichtung zu Grunde gelegt, so erhöht sich dessen Zustimmungswert unter sehr wichtig um 5, 3 Prozentpunkte. Insbesondere Kommunen unter 5000 EW sehen dieses Hemmnis als wichtig an. Wird jedoch der Zersiedlungsindex angesetzt, so fällt dieser Wert wieder, wodurch impliziert werden kann, dass dieses Hemmnis zwar von einwohnerschwachen Kommunen, jedoch nicht von tendenziell zersiedelten Kommunen, also deren bebauter Siedlungskörper im Raum gestreut ist, getragen wird. Wird die Kategorie eher wichtig hinzugenommen, so relativiert sich diese Betrachtung jedoch, sodass im Allgemeinen rund 52 \% der Befragten unter Hinzunahme des Zersiedelungsindikators diesem Hemmnis zustimmen, wohingegen dies in der ungewichteten Variante nur $47 \%$ sind.

Das Vergabeverhalten verbleibt unabhängig der Gewichtung auf einem konstant hohen Niveau mit über 60 \%allgemeiner Zustimmung. In der Tendenz schätzen Kommunen mit geringer Breitbandverfügbarkeit das Vergabeverhalten als wichtiges Hemmnis ein. Zudem sehen gerade Kommunen kleiner 5000 EW das Vergabeverhalten als Hemmnis an. ${ }^{39}$ Hierdurch zeigt

39 Signifikanzniveau: $5 \%$. 
sich, dass die gesamte kommunale Familie das Vergabeverhalten als eher problematisch ansieht. Dies wird durch die Pflicht zur europaweiten Ausschreibung untermauert. Das Antwortverhalten der Kommunen mit geringer Breitbandverfügbarkeit sowie von Kommunen mit weniger als $5000 \mathrm{EW}$ weisen eine ähnliche Tendenz auf, wie bereits bei der Einschätzung zum Vergabeverhalten. Rund 42 \%sehen dies im Allgemeinen als Hemmnis an, wobei dieser Wert in der Einwohnergewichtung zunimmt. Vor dem Hintergrund kleinerer Verwaltungen in einwohnerschwachen Kommunen ist eine europaweite Ausschreibung als verwaltungsintensiver zu interpretieren und bindet somit längerfristig personelle Kapazitäten, die, wie weiter oben gezeigt, sich in einer angespannten Situation befinden. J edoch muss festgehalten werden, dass dieses Hemmnis als eher nachrangig im Vergleich zum Vergabeverhalten beschrieben werden kann, da durchgängig niedrigere Zustimmungswerte erreicht werden.

Die kommunale Familie stuft den Breitbandausbau als dringlich ein und sieht dies folglich nicht als Hemmnis: Ein Problembewusstsein besteht somit. Insbesondere kleinere Kommunen haben die Dringlichkeit des Breitbandausbaus erkannt. Kommunen kleiner als $10000 \mathrm{EW}$, Bauämter mit vergleichsweise wenigen VZÄ sowie Kommunen mit einer geringeren Breitbandverfügbarkeit sehen in der Tendenz dies als geringes Hemmnis an. In Anbetracht der Tatsache, dass wie weiter oben beschrieben, Breitband als Standortfaktor angesehen werden kann, ist festzuhalten, dass sich speziell kleinere Kommunen dieser Tatsache im hohem Umfang bewusst sind.

Ein inhaltlicher Punkt, aus den bereits in Bezug auf die Tiefbaukoordinierung vermehrt eingegangen worden ist, bezieht sich auf die Fördermittelprogrammatik. Auch im Bereich des Breitbandausbaus können Hindernisse identifiziert werden, die einer adäquaten Nutzung der verfügbaren Fördermittel entgegenstehen. Wie Abbildung 23 zeigt, wird insbesondere die starke Zeitverzögerung im Fördermittelprozess als hinderlich angesehen. In der ungewichteten Variante stimmen dieser Aussage insgesamt rund $67 \%$ der Befragten zu. Dieser Wert erhöht sich in der einwohnergewichteten Darstellung um zehn Prozentpunkte. Zudem nimmt der Neutralitätsgrad im Zuge der Gewichtung um rund neun Prozentpunkte ab, wodurch geschlussfolgert werden kann, dass dies im Besonderen ein Problem für kleinere Kommunen darstellt. 
Abbildung 23: Stimmen Sie aus Sicht Ihrer Kommune zu, das die folgenden Hindernisse im Rahmen der Fördermittelprogrammatik, beispielsweise der Digitale Offensive Sachsen, bestehen?

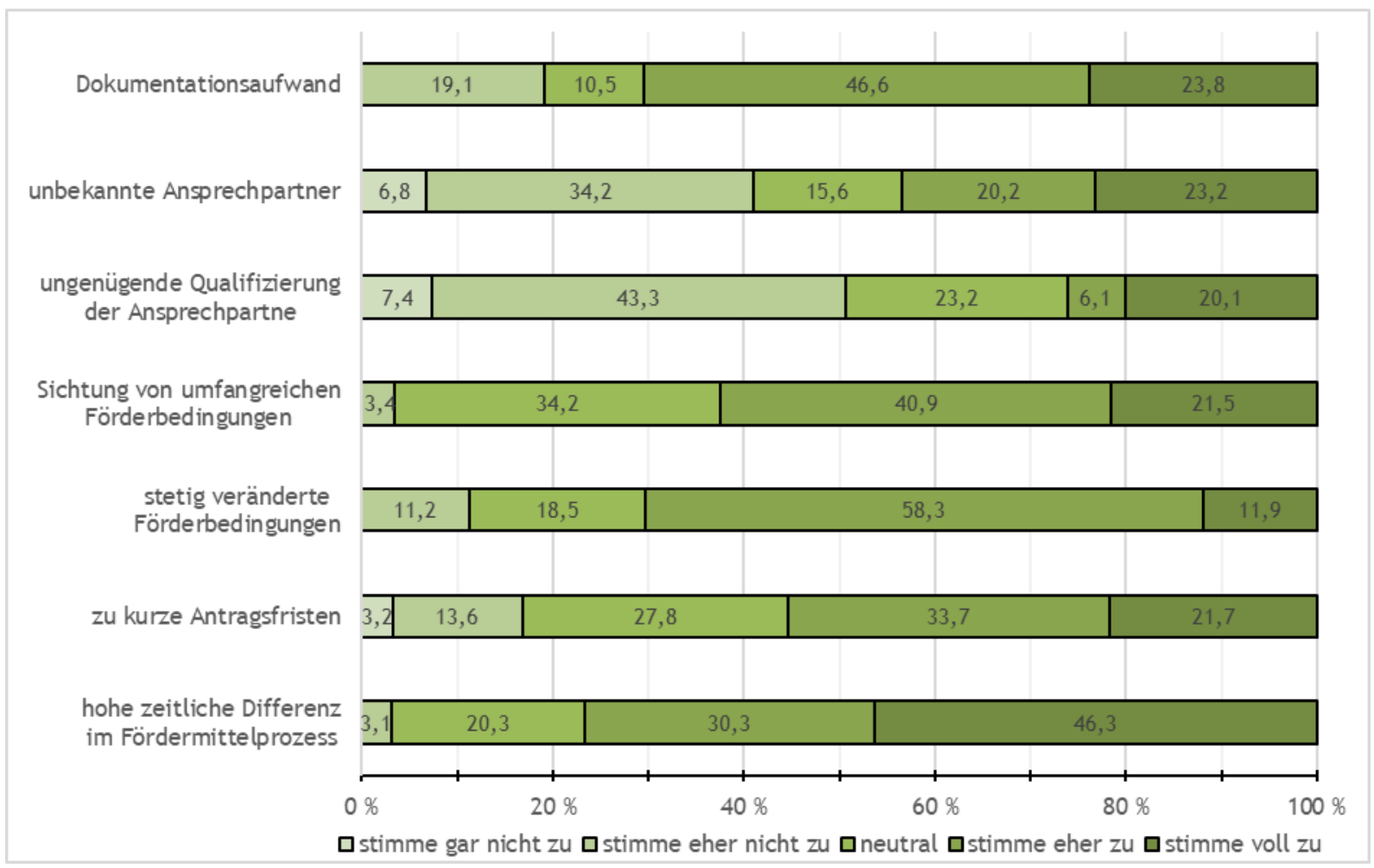

Quelle: Eigene Darstellung; eigene Berechnung, $\mathrm{N}=27$.

Einheitlich bewerten die Kommunen den Dokumentationsaufwand, der mit den Fördermitteln im Bereich des Breitbandausbaus einhergeht. Rund $68 \%$ aller Befragten teilen die Ansicht, dass der Dokumentationsaufwand ein Hindernis darstellt. In der Tendenz stimmen eher Kommunen zu, die kleiner $10000 \mathrm{EW}$ sind sowie über überproportional viele investive Schulden je EW verfügen.

Unklare Ansprechpartner scheinen hingegen kein sonderlich großes Problem darzustellen, wobei somit geschlussfolgert werden kann, dass die übergeordneten Instanzen klar benannt sind und Zuständigkeiten entsprechend kommuniziert werden. Lediglich eine Teilgruppe weicht im Antwortverhalten ab: Kleinere Kommunen mit weniger als $10000 \mathrm{EW}^{40}$. Dahingehend scheinen Informationsungleichgewichte vorzuliegen. Dies kann wiederum als Indiz für die starke Belastung der Bauverwaltungen in kleineren Gemeinden gewertet werden. Dagegen wird die Qualifikation der Ansprechpartner nach Meinung der kommunalen Ebene als hinreichend gut eingeschätzt, um bei Fragen und Problemen eine Auskunft erteilen zu können.

40 Signifikanzniveau: $5 \%$. 
Im Hinblick auf die Sichtung der umfangreichen Förderbedingungen stimmen rund $63 \%$ in der gewichteten und ungewichteten Variante der Aussage zu, dass die Überprüfung und Begutachtung der Bedingungen zur Nutzung von bestehenden Fördermitteltöpfen ein Problem darstellen, wobei diese Aussage unabhängig der kommunalen Größe geteilt wird. Dies wird durch den Fakt untermauert, dass die sich stetig verändernden Förderbedingungen ebenfalls als gravierendes Hemmnis erkannt worden sind. Rund 65 \% teilen diese Auffassung, wobei auch hier nur ein kleiner Unterschied (+ 5 Prozentpunkte) zwischen der Einwohnergewichtung und der ungewichteten Darstellungsweise vorliegt, der sich in der Tendenz durch kleinere Kommunen unter 10000 EW sowie Kommunen mit geringer Breitbandverfügbarkeit erklären lässt.

Die vorliegenden Ergebnisse zu diesem Aspekt zeigen, dass die Probleme mehrheitlich nicht bei den übergeordneten (Bewilligungs-)Stellen und deren Expertise zu sehen sind. Vielmehr ist die bestehende Fördermittelprogrammatik per se als Problem identifiziert worden. Hierbei ist der gesamte Fördermittelprozess als Hemmnis benannt worden. Durch umfangreiche Förderbedingungen werden langfristig personelle Kapazitäten in den öffentlichen Verwaltungen gebunden, wodurch diese der weiterführenden Verwaltungstätigkeit nicht zur Verfügung stehen. Dieses Problem wird sogar noch verstärkt, indem sich die Förderbedingungen stetig wandeln, wodurch dieser Aufwand nicht als einmalig, sondern als persistent zu verstehen ist. Hierdurch müssen dauerhaft personelle Mittel für die Begutachtung von Fördermittelbedingungen vorgehalten werden, die im weiterführenden Verwaltungsprozess fehlen. Dies verstärkt die allgemein als problematisch zu beschreibende personelle Ausstattung in den kommunalen Verwaltungen. Zudem ist bis zu diesem Zeitpunkt offen, ob die Fördermittel überhaupt beantragt werden können (Förderbedingungen) oder ob letztlich der Fördermittelantrag Erfolg haben wird (limitierter Fördermitteltopf), wodurch im ökonomischen Sinne mit der Analyse von Fördermittelbedingungen von versunkenen Kosten gesprochen werden kann. Sofern sogar ein positiver Fördermittelbescheid vorliegt, verzögert sich dessen Auszahlung augenscheinlich, da die starke Zeitverzögerung im Fördermittelprozess als maßgebliches Hindernis genannt worden ist. Diese Dynamiken zeigen, dass der Fördermittelprozess aus Sicht der Kommunen bisher vor allem fixe Kosten verursacht und diesen Kosten nur unsichere Erträge (Fördermitteleinnahmen) entgegenstehen. Für die kommunale Ebene, die im Allgemeinen auf Fördermittel zur Finanzierung öffentlicher Daseinsvorsorge angewiesen ist, ist diesem Sachverhalt ein hohes Maß an Unsicherheit inhärent, bei dem die Risiken als hoch einzuschätzen sind.

Vor diesem Hintergrund wurde erfragt, wie verschiedene Instrumente zur Förderung des Breitbandausbaus abseits der Fördermittelproblematik eingeschätzt werden und welche 
Ebene diese hauptsächlich durchführen sollte. Hierbei wird deutlich, dass die interkommunale Kooperation als effektives Instrument eingeschätzt wird, um den kommunalen Breitbandausbau zu forcieren. Dabei nimmt diese Meinung in der Einwohnergewichtung zu. Dies lässt sich insbesondere durch das Antwortverhalten der Gemeinden unter 5000 EW erklären. Gerade einwohnerschwache Kommunen sehen das Instrument der interkommunalen Kooperation als zentral an. Auch die externe Beratung sowie die Qualifizierungs- und Weiterbildungsmaßnahmen erreichen über die Einwohnergewichtung hohe Zustimmungswerte. Dies ist jedoch vor dem Hintergrund nicht verwunderlich, dass einwohnerschwache Kommunen oftmals kleinere öffentliche Verwaltungen haben, sodass entweder Expertise extern hinzugezogen oder intern erschlossen werden muss. Dies lässt sich damit belegen, dass in der Tendenz besonders Kommunen kleiner 10000 EW sowie Bauämter mit wenig VZÄ besonders stark die Effektivität in ihrem Antwortverhalten betonen. Statistisch signifikant ist zudem die moderate Korrelation zwischen Kommunen mit geringer Breitbandverfügbarkeit: Diese Teilgruppe der befragten Kommunen, welche mit dem Breitbandausbau vor Ort nicht voran kommt, weist eine deutlich höhere Einschätzung der Effektivität von externer Beratung und Weitbildungsmaßnahmen auf. ${ }^{41}$

Abbildung 24: Wie schätzen Sie Instrumente zur Förderung des Breitbandausbaus hinsichtlich ihrer Effektivität ein?

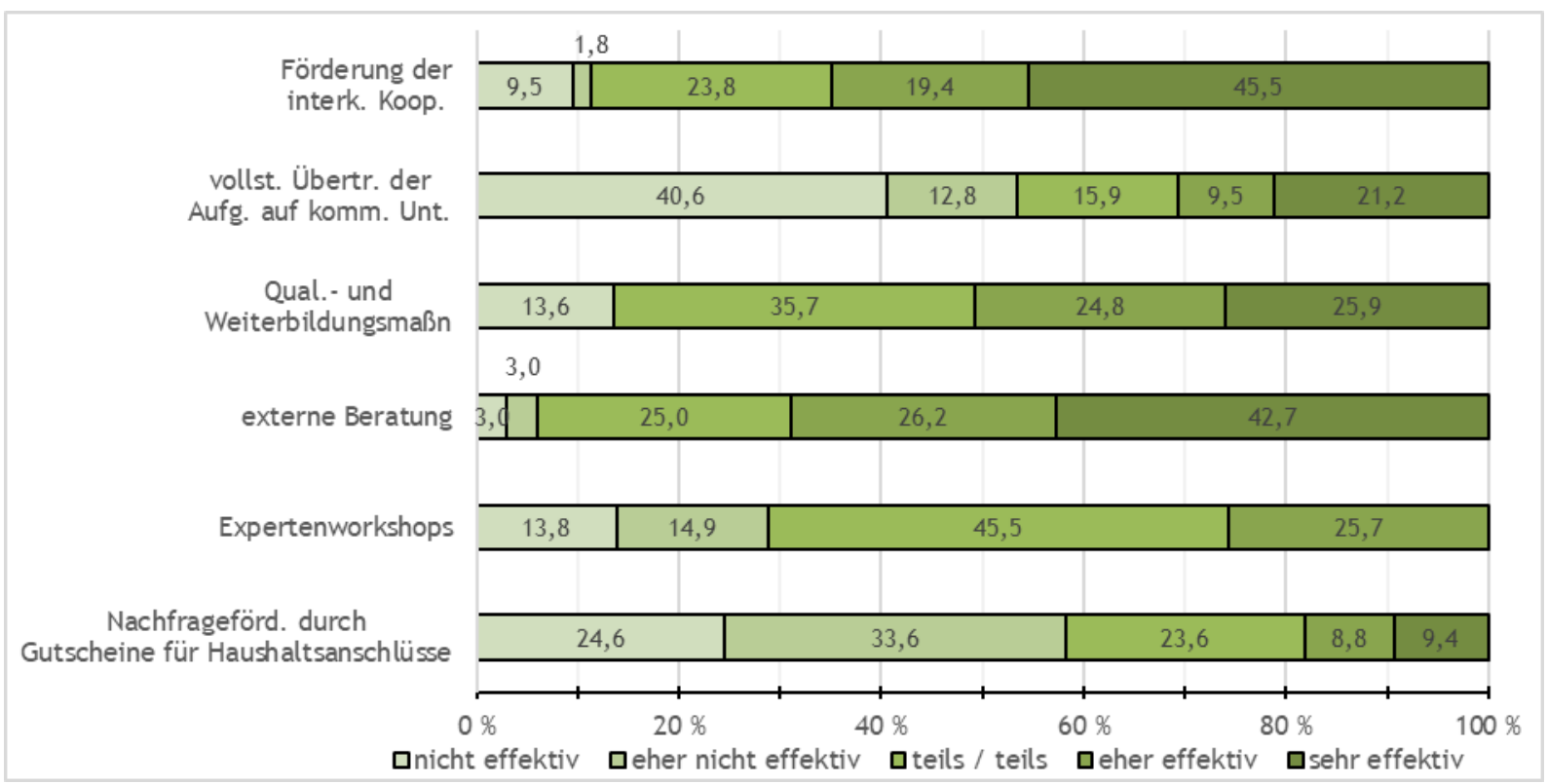

Quelle: Eigene Darstellung; eigene Berechnung, $\mathrm{N}=27$.

Die vollständige Übertragung der Aufgaben auf ausgelagerte Einheiten, wie etwa auf kommunale Unternehmen, wird von den Befragten interessanterweise nicht als ernstzuneh-

41 Signifikanzniveau: $5 \%$ **. 
mende Lösungsoption beschrieben. Dies zeigt sich einerseits durch die geringen Zustimmungswerte und andererseits durch den relativ kleinen Anteil neutraler Antworten. Dies ist auch unabhängig von der Gemeindegröße. Folglich ist dies aus Sicht der kommunalen Bauverwaltung kein probater Ansatz.

Auch Expertenworkshops werden vergleichsweise als eher kritisch angesehen. Gerade einmal 25, $7 \%$ halten diese Variante für effektiv, wobei 45, $5 \%$ der Befragten unentschlossen sind. Dies ist jedoch abhängig von den Iokalen Verwaltungskapazitäten. Städte und Gemeinden unter $10000 \mathrm{EW}^{42}$ sowie Bauämter mit wenig Personal ${ }^{43}$ bewerten dieses Instrument aus ihrer Sicht als deutlich effektiver als die übrigen Teilgruppen. Die Belastung einer stark beanspruchten Verwaltung lässt es für diese Kommunen attraktiver erscheinen, externe Unterstützung einzuholen, um Lösungen zu finden.

Die Option, die Nachfrage durch Gutscheine für Haushaltsanschlüsse zu fördern, trifft auf ein gemischtes Echo. Der Großteil der Befragten sieht eine solche Form der Nachfrageförderung eher kritisch. Interessanterweise stellt dies einen leichten Dissens zu den Ergebnissen aus Abbildung 19 dar. Dort wurde nach den Faktoren für einen erfolgreichen Breitbandausbau gefragt, wobei neben Unternehmensaspekten auch die privaten Haushalte auf der Nachfrageseite als wichtiger Einflussfaktor genannt worden sind. Aus Sicht der Kommunen erscheint es allerdings erfolgversprechender, auf angebotsseitige Effekte zu setzen, d. h., den Ausbau aktiv zu befördern, statt durch eine gestärkte Nachfrage auf privatwirtschaftliche Ausbauaktivitäten zu hoffen.

Neben der Frage nach der Effektivität wurde zudem erfragt, welche Verwaltungsebene für das entsprechende Instrument zuständig sein sollte. Abbildung 25 gibt hierzu einen Überblick. 


\section{Abbildung 25: Welche Verwaltungsebene sollte diese Maßnahme aus Sicht Ihrer Kommune} hauptsächlich durchführen?

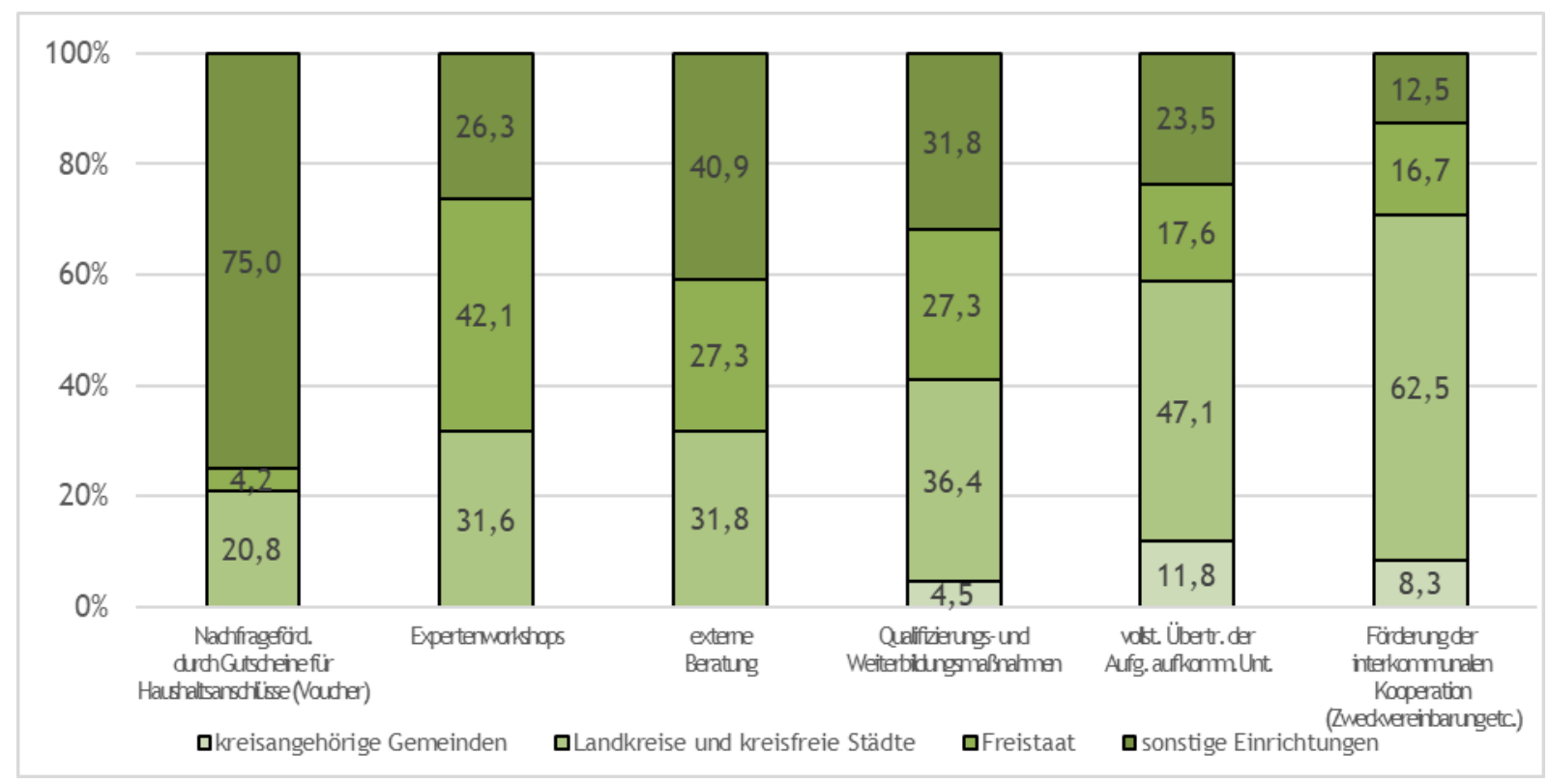

Quelle: Eigene Darstellung; eigene Berechnung, $\mathrm{N}=27$.

Die Nachfrageförderung durch Gutscheine für Haushaltsanschlüsse wird mehrheitlich bei sonstigen Einrichtungen gesehen. Nur ca. $20 \%$ aller Befragten halten die Landkreise und kreisfreien Städte hier für die geeignete staatliche Ebene.

Hingegen werden die Expertenworkshops mit rund $42 \%$ dem Freistaat zugerechnet, wobei mit rund zehn Prozentpunkten Abstand die Landkreise und kreisfreien Städte folgen. Einen Vergleichbaren Wert von rund 31 \% erreichen die Landkreise und kreisfreien Städte bei der externen Beratung. J edoch werden auch hier die sonstigen Einrichtungen mit rund $41 \%$ als maßgebend angesehen. Die Qualifizierungs- und Weiterbildungsmaßnahmen werden mehrheitlich mit rund $36 \%$ als Aufgabe der Landkreise und kreisfreien Städte verstanden, wobei mit zirka 32 \%die sonstigen Einrichtungen folgen. Sofern Aufgaben vollständig auf eine übergeordnete Ebene übertragen werden sollten, so würde mit rund $47 \%$ diese Aufgabe den Landkreisen und kreisfreien Städten zugeordnet werden. Auch im Rahmen der interkommunalen Kooperation werden die Landkreise und kreisfreien Städte mit 62,5\% als geeignete Verwaltungsebene identifiziert.

Werden beide Elemente miteinander vereint, so ergibt sich folgendes Bild: Zur Förderung des Breitbandausbaus werden letztlich die interkommunale Kooperation (1), die externe Beratung der öffentlichen Verwaltung (2) und Qualifizierungs- und Weiterbildungsmaßnahmen (3) genannt. Die Instrumente (1) und (3) sollen hierbei durch die Landkreise und kreisfreien Städte erfolgen, während die externen Beratungsleistungen durch die sonstigen Einrichtungen zu erbringen wären. Es zeigt sich somit, dass neben der Nutzung von Größenvorteilen (interkommunale Kooperation) vor allem die Wissensaneignung für die kommunale Ebene im 
Vordergrund steht. Letztlich wirken die Kommunen mit der ihnen zugeordneten Aufgabe überfordert. Deren Lösung ist es, vermehrt mit anderen Kommunen zu kooperieren und sofern möglich, externe Unterstützung zu nutzen. Es verstetigt sich der Eindruck, dass die Aufgabe von einer anderen übergeordneten Ebene hätte weiter unterstützt werden können.

Um den Wissenstransfer zu erleichtern, hat das Gesetz zur Erleichterung des Ausbaus digitaler Hochgeschwindigkeitsnetze (DigiNetzG) Umsetzungshilfen erlassen, die in Bezug auf ihren Nutzen eingeschätzt werden sollten. Abbildung 26 stellt hierbei das Antwortverhalten der befragten Kommunen dar. Auf eine gewichtete Darstellung wurde verzichtet, da hierdurch keine relevante Verschiebung im Antwortverhalten steht - die befragten kommunen haben über alle Kriterien hinweg homogen geantwortet.

Abbildung 26: Für wie hilfreiche schätzen Sie die Umsetzungshilfen des Gesetzes zur Erleichterung des Ausbaus digitaler Hochgeschwindigkeitsnetze (DigiNetzG) ein?

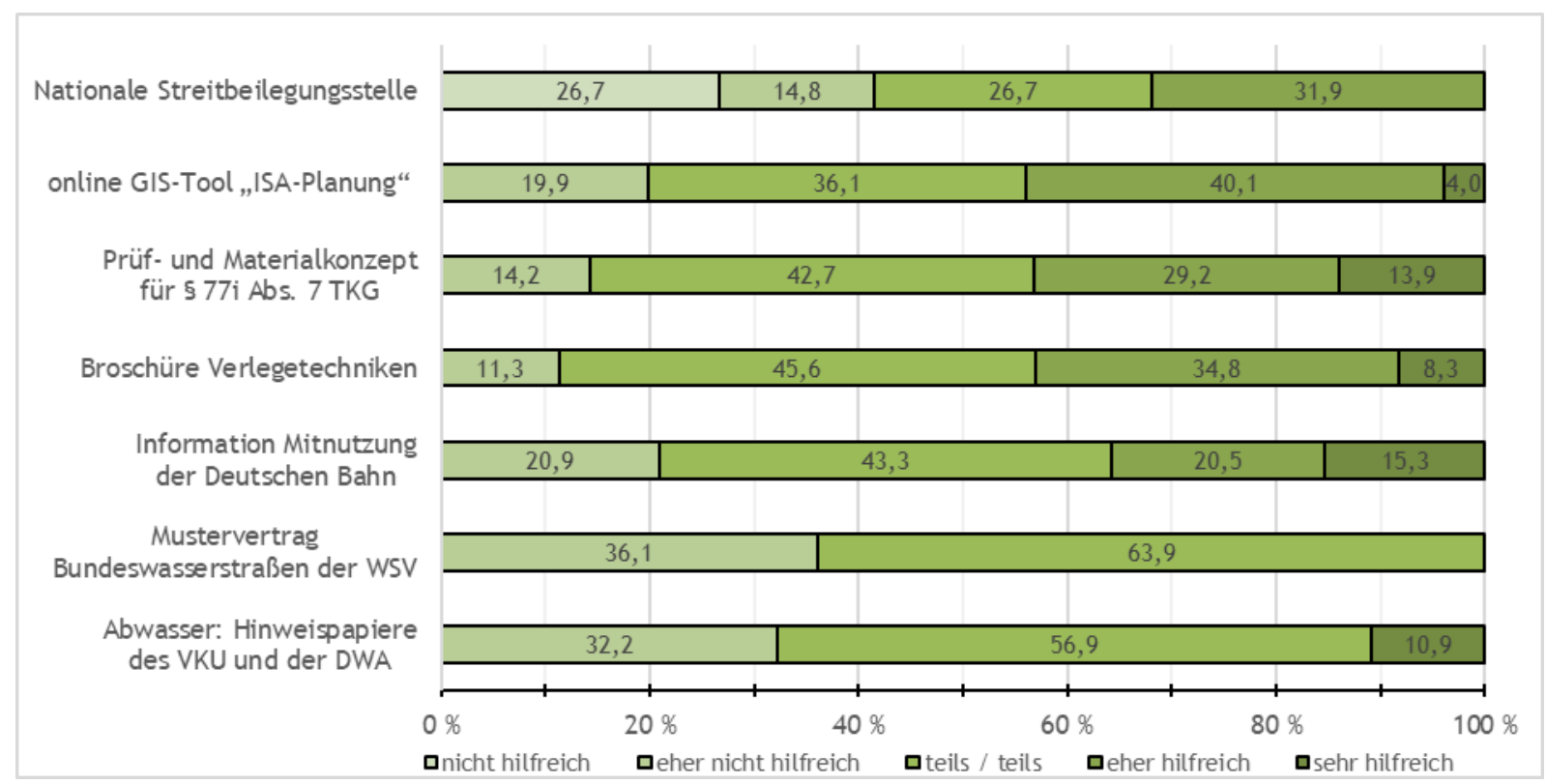

Quelle: Eigene Darstellung; eigene Berechnung, $\mathrm{N}=19$.

Hierbei stechen das Prüf- und Materialkonzept für §77i Abs. 7 TKG, die Broschüre zu den Verlegetechniken sowie die Informationen zur Mitnutzung der DEUTSCHEN BAHN AG positiv hervor. Auch das online GIS-Tool "ISA-Planung" und die Hinweispapiere des VKU und der DWA werden als hilfreich eingeschätzt. Die einzige Umsetzungshilfe, die die schlechteste Bewertung „nicht hilfreich“ erhalten hat, ist die nationale Streitbeilegungsstelle. Auch der Mustervertrag Bundeswasserstraßen der WSV wird als nicht hilfreich eingeschätzt.

Da neben der internen Weiterbildung und Qualifizierung auch die externe Beratung als sehr effektives Instrument eingeschätzt worden ist, wurde zudem erfragt, für wie hilfreich die Zusammenarbeit mit folgenden Partnern im Bereich der Unterstützung, Planung oder Umsetzung eines Breitbandausbauprojektes angesehen wird. Abbildung 27 gibt hierzu einen Überblick. 
Staatlich oder öffentlich geförderte Beratungsangebote werden mit zirka $47 \%$ als hilfreich angesehen, wobei in der Einwohnergewichtung diese Zustimmungsrate um 14 Prozentpunkte zurückgeht - folglich sind kleinere Kommunen bezüglich öffentlich geförderten Beratungsangeboten eher zurückhaltend eingestellt. Davon abweichend erachten Kommunen mit wenig VZÄ in den Bauämtern staatlich oder öffentlich geförderte Beratungsangebote als hilfreich. ${ }^{44}$ Wird der Zersiedlungsindikator als Gewichtungsgrundlage herangezogen, so zeigt sich ein erneuter Anstieg der Zustimmung, wodurch geschlussfolgert werden kann, dass zwar einwohnerschwache Kommunen staatliche bzw. öffentlich geförderte Beratungsangebote eher kritisch sehen, jedoch insbesondere zersiedelte Kommunen dieses Instrument als hilfreich ansehen. Kommunen, die bereits ein oder mehrere Projekte im Bereich Breitbandausbau durchgeführt haben, bewerten staatlich bereitgestellte Beratungsangebote als hilfreich. ${ }^{45}$ So profitiert die Begleitung oder eigenständige Durchführung von Breitbandprojekten in Sachsen von den Beratungsangeboten des Freistaats.

Private Beratungsunternehmen oder Planungsbüros genießen indes ungewichtet, wie auch über die beiden Gewichtungsindizes, einen hohen Stellenwert. Auch hier weist das Antwortverhalten von Bauämtern mit weniger VZÄ auf eine deutlich hilfreichere Einschätzung hin. ${ }^{46}$ Dies korrespondiert nicht zuletzt auch mit der Aussage der Kommunen, dass externe Expertise als wichtiger Faktor für einen erfolgreichen Breitbandausbau angesehen werden kann. Kommunen, die bereits ein oder mehrere Proj ekte im Bereich Breitbandausbau durchgeführt haben, bewerten in der Tendenz die Zusammenarbeit mit privaten Unternehmen als hilfreich. Demzufolge sind kleinere Bauämter auf die Unterstützung von Beratungsunternehmen oder Planungsbüros angewiesen. Aus ihrer Erfahrung wird die Zusammenarbeit als produktiv eingeschätzt.

Signifikanzniveau: $1 \%$ \%**.

45 Signifikanzniveau: $5 \%$ * .

46 Signifikanzniveau: $1 \%$ ***. 
Abbildung 27: Für wie hilfreich schätzen Sie die Zusammenarbeit mit den folgenden Partnern für Unterstützung, Planung oder Umsetzung eines Breitbandausbauprojektes ein?

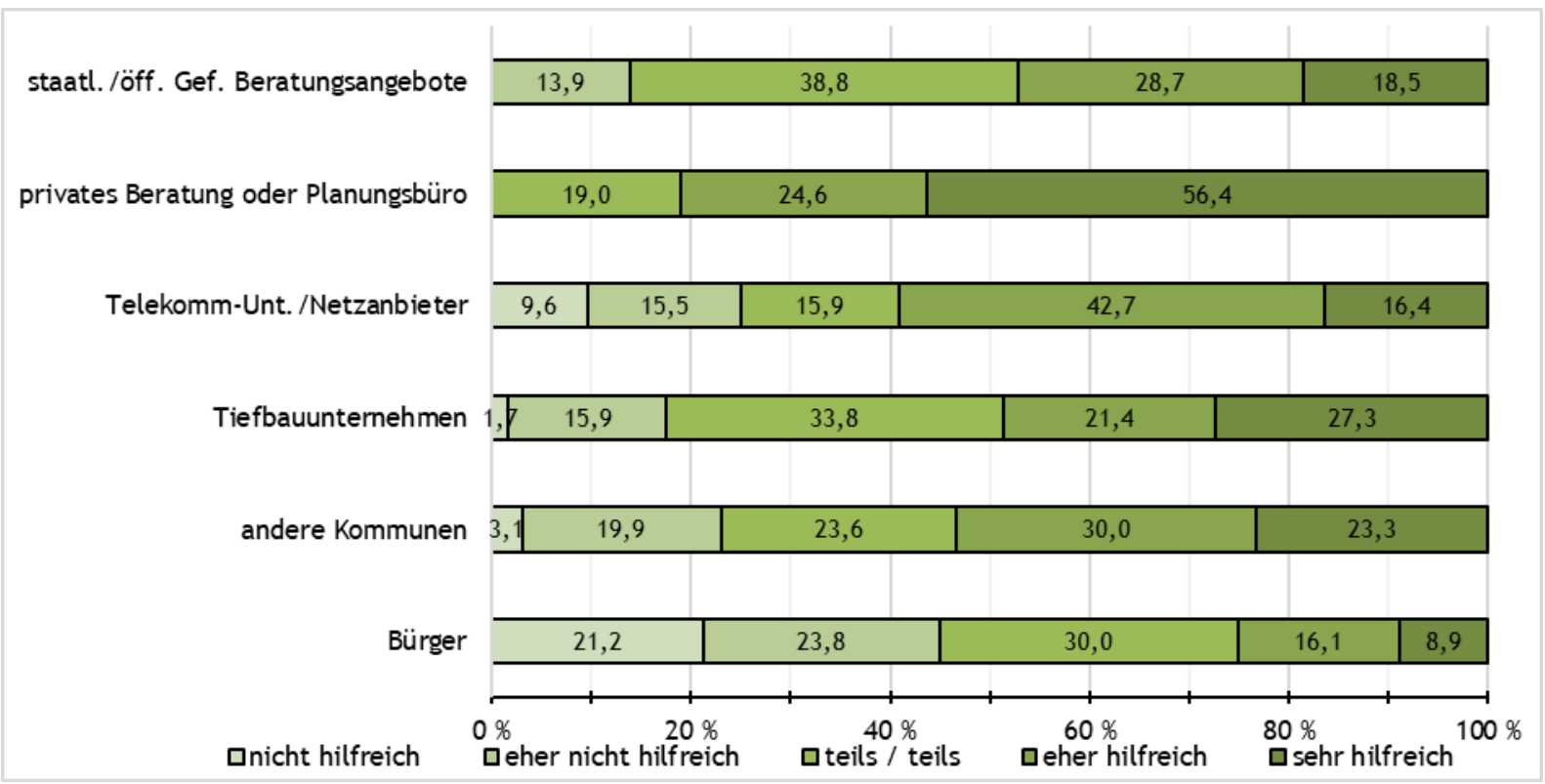

Quelle: Eigene Darstellung; eigene Berechnung, $\mathrm{N}=28$.

Telekommunikationsunternehmen und Netzanbieter erreichen zwar geringere Zustimmung in der höchsten Kategorie, allerdings ist die allgemeine Einschätzung sehr positiv: 59 \% der Befragten schätzen diese als hilfreich ein. Insbesondere Kommunen unter $5000 \mathrm{EW}$ schätzen die Zusammenarbeit mit Tiefbauunternehmen als hilfreich ein, da diese nicht in der Lage sind, die Tiefbaumaßnahmen zu planen und durchzuführen. ${ }^{47}$

Bei der Bewertung der Relevanz und Effektivität eines erfolgreichen Breitbandausbaus wurde die interkommunale Kooperation hervorgehoben. Dies spiegelt sich ebenfalls im Hinblick auf die Frage der Zusammenarbeit wider. In der Kategorie „andere Kommunen“ sind hohe Zustimmungswerte erreicht worden, die sich beispielsweise in der Einwohnergewichtung auf rund 70 \%einpendeln. Dabei ist kaum verwunderlich, dass insbesondere kleinere Kommunen die interkommunale Kooperation als wichtiges Hilfsmittel einschätzen, da hierdurch Größenvorteile gehoben und Spezialisierungseffekte gewonnen werden können.

Die Bürgerbeteiligung wird im Vergleich zu den anderen Institutionen und staatlichen Einrichtungen dagegen als eher nachrangig wahrgenommen. Dies ist im Hinblick auf die zuvor artikulierte Wichtigkeit der privaten Haushalte für einen erfolgreichen Breitbandausbau jedoch zu hinterfragen. Augenscheinlich werden die privaten Haushalte in ihrer Rolle als Konsumenten als wichtige Treiber des Breitbandausbaus identifiziert, jedoch scheinen Bürgerbeteiligungsprozesse nur als bedingt hilfreich gesehen zu werden.

47 Signifikanzniveau: $5 \%$ **. 


\section{$4 \quad$ Zusammenfassende Bemerkungen}

Hinsichtlich der Tiefbaumaßnahmen lassen sich abschließend einige zentrale Erkenntnisse feststellen. Die Bündelung von Tiefbaumaßnahmen wird im kommunalen Kontext in Sachsen grundsätzlich kaum fokussiert. Es bleibt festzuhalten, dass zersiedelte und kleinere Kommunen häufiger ihre Tiefbaumaßnahmen bündeln. Als großes Hindernis der Koordinierung im Tiefbau konnte das z.T. gering vorhandene Personal in den Bau- und respektive Hauptämtern bestätigt werden. Auch hier sind insbesondere kleine Kommune betroffen. Darüber hinaus bestehen aus Sicht kleinerer Kommunen unklare Zuständigkeiten und externe Informationsasymmetrien, die zu einer Koordinierungsbremse führen. Hingegen leiden größere Kommunen eher unter internen Informations- und Kommunikationsproblemen. Gemeinsam fordern große wie kleine Kommunen vereinfachte Genehmigungsverfahren für Tiefbaumaßnahmen. Die Digitalisierung der Baukoordinierung erscheint vor allem für kleine Städte und Gemeinden von Bedeutung. Diese fordern ebenfalls höhere Förderanteile in den entsprechenden Förderprogrammen und höhere Fördersätze im Finanzausgleich, was auf weitere strukturelle Probleme hindeutet. Kommunen, die bereits ihre vorhandenen Leitungen dokumentieren, nutzen dafür größtenteils GIS-basierte Karten und stellen die Daten auf Anfrage kostenlos oder gegen Gebühr für Dritte bereit.

Die These, dass angebots- mehr als nachfrageseitige Faktoren für den erfolgten Breitbandausbau relevant sind, kann mit den Ergebnissen der Umfrage bestätigt werden. Überdies herrscht in den Kommunen die Einschätzung, dass der Breitbandausbau eher für Privathaushalte und Unternehmen, weniger für z. B. die Digitalisierung der Verwaltung wichtig sei. Die Hälfte der befragten Kommunen gab an, über Erfahrungen mit dem kommunalen Ausbau von Breitband, im Sinne eines Wirtschaftlichkeitslücken- oder Betreibermodells, zu verfügen. Weiterhin konnte herausgearbeitet werden, dass das Vergabeverhalten über alle Größenklassen hinweg als ein Hemmnis wahrgenommen wird. Nicht überraschend ist, dass die Entfernung zwischen einzelnen Ortslagen tendenziell kleine und zersiedelte Kommunen hemmt, den Breitbandausbau voranzutreiben. Es bleibt zu betonen, dass die angebotsseitigen Faktoren des Breitbandausbaus wenig elastisch sind und sich daher nicht im Laufe der Zeit "von selbst Iösen“, sondern ein aktives Handeln seitens der zuständigen Gebietskörperschaften erfordert.

Polarisierend wirkt der zeitliche Verzug des Erhalts von Mitteln bei Förderprogrammen, worauf besonders kleine Kommunen hinweisen. Der Dokumentationsaufwand bei Förderprogrammen wird durchgängig beanstandet. Dennoch leiden auch darunter mehr kleine und verschuldete Kommunen, die aus eigener Sicht die bürokratischen Pflichten nicht stemmen können. Informationsprobleme bei den Förderprogrammen liegen ebenfalls nur bei kleinen Kommunen vor. 
Als effektives Instrument, um den kommunalen Breitbandausbau zu fördern, wird die interkommunale Kooperation genannt. Diese sollte laut Meinung der befragten Kommunen durch die Landkreise und kreisfreien Städte unterstützt werden. Kleine Kommunen, solche mit wenig VZÄ im Bauamt, oder solche mit bisher wenig erfolgten Breitbandausbau, wünschen sich mehr Qualifizierung und Weitbildungen in diesem Bereich. Dies könnte ebenfalls durch Landkreise bzw. kreisfreie Städte oder externe Einrichtungen erbracht werden. Staatlich oder im weiteren Sinne öffentlich geförderte Beratungsangebote werden insbesondere von größeren Kommunen mit wenig Personal in den Bauämtern oder zersiedelter Struktur gewünscht - trotz oder gerade wegen bestehender Erfahrungen mit kommunalen Breitbandausbau in diesen Kommunen. Auch private Beratungsunternehmen oder Planungsbüros werden vor allem von Kommunen mit wenig Personal in den Bauämtern und Erfahrung mit kommunalen Breitbandprojekten als wichtige Partner eingeschätzt. Die bestehenden Hilfen des DigiNetzG werden als sachdienlich eingeschätzt, wobei die Streitbeilegungsstelle nicht als hilfreich angesehen wird. Eine vollkommene Übertragung dieser kommunalen Aufgabe auf ausgelagerte Einheiten wird durchgängig abgelehnt.

Abschließend ist festzuhalten, dass bereits verbaute und neu zu installierende - insbesondere digitale - Infrastruktur in Sachsen noch nicht durchgängig zusammengedacht werden. Hierdurch können potentielle Synergien nicht ausgeschöpft werden, wodurch unter anderem zusätzliche (Mehr-)Kosten bestehen. Zudem ist durch die häufigeren Bauphasen mit Einschränkungen der öffentlichen Leistungserbringung zu rechnen, wodurch sich für Bürger und die am Standort angesiedelten Unternehmen negative Effekte ergeben können. Somit stellt eine effektive Tiefbaukoordinierung einen wesentlichen Bestandteil für die öffentliche Leistungserbringung in Kommunen dar. Hierzu gilt es, durch gezielte Angebote auf Ebene der Landkreise sowie fördernd durch den Freistaat gegenzusteuern. Insbesondere bei dem Aufkommen neuer Belastungen durch zusätzliche Belastungen, wie beispielsweise dem geförderten Breitbandausbau oder bei technischen Neuerungen, wie der Digitalisierung der Baukoordinierung, gilt es unterstützend aktiv zu werden. 


\section{Literaturverzeichnis}

Bender, Christian/ Mengs, Christoph (2020): Mobilität in Sachsen - Status Quo, Herausforderungen und Handlungsempfehlungen der kommunalen Verkehrsinfrastruktur, KOMKIS Analyse, Nr. 13, Leipzig.

BÖCKER, JENS (2019): Breko Marktanalyse 19, Bundesverband Breitbandkommunikation e. V. (Breko), Böcker Ziemen Management Consultants GmbH \& Co. KG, Berlin/ Bonn.

BREITBANDKOMPETENZZENTRUM SACHSEN (2019): Breitbandförderung: Workshop für die Betreibermodelle in Sachsen vom 20.06.2019, abrufbar unter: https:// www. digitale. offensive. sachsen. de/ 15114. html.

BUNDESMINISTERIUM FÜR VERKEHR UND DIGITALE INFRASTRUKTUR (BMVI) (2019): Bericht zum Breitbandatlas, Teil 1: Ergebnisse, Stand 06/2019, Berlin, abrufbar unter: https: / / www. bmvi. de/ SharedDocs/ DE/ Anlage/ DG/ Digitales/ bericht-zum-breitbandatlasmitte-2019-ergebnisse. pdf?_blob=publicationFile, 04.11.2020.

BUNDESREGIERUNG (2020): Glasfaserausbau und Fortentwicklung der staatlichen Förderung. https: / / www. bundesregierung. de/ breg-de/ themen/ digital-made-in-de/ glasfaserausbauund-fortentwicklung-der-staatlichen-foerderung-1546612, 04.11. 2020.

DeUTSCHER BUndeSTAG (2019): Breitbandausbau im Freistaat Sachsen, Antwort der Bundesregierung auf die Kleine Anfrage der Abgeordneten Stephan Kühn (Dresden), Matthias Gastel, Stefan Gelbhaar, Daniela Wagner und der Fraktion BÜNDNIS 90/DIE GRÜNEN vom 10.07.2019, Drucksache 19/11464, abrufbar unter: http://dip21. bundestag. de/ dip21/ btd/ 19/ 114/ 1911464. pdf, 30.09.2020.

EBERMANN, ERWIN (2010): Grundlagen statistischer Auswertungsverfahren, online verfügbar unter: $\quad$ https:/ / www. univie. ac. at/ ksa/ elearning/ cp/ quantitative/ quantitative-101. html, 29.01.2020.

Handschuh, Alexander/ Hornbostel, Lorenz/ Nerger, Michael/ Salden, J anina/Tillack, DÉSIRÉE/ WITTPAHL, VOLKER (2019): Zukunftsradar Digitale Kommune - Ergebnisbericht zur Umfrage 2019, Institut für Innovation und Technik, Deutscher Städte- und Gemeindebund, Berlin.

IÖR-MONITOR (2020): Indikatorenblatt Gewichtete Zersiedlung, online verfügbar unter: http:/ / www. ioer-monitor. de/ ?id=44\&ID_IND=D01KG, 03.12.2020.

J AEger, J.A.G./ SCHWick, C./ HenNig, E. I./ BehnisCH, M./ SChWARZAK, M./ KRÜGER, T./ SOUKUP, T./ ORLitova, E./ NaZARniA, N./ Kienast, F. (2018): Messung der Zersiedelung ermöglicht Monitoring und stärkere Berücksichtigung in der Planung. In: Behnisch, M.; Kretschmer, O.; Meinel, G.: Flächeninanspruchnahme in Deutschland - Auf dem Wege zu einem besseren Verständnis der Siedlungs- und Verkehrsflächenentwicklung, Springer Spektrum, Berlin.

KRATZMAnN, AleXANDER (2020): Interkommunale Zusammenarbeit im Freistaat Sachsen - Eine Bestandsaufahme, KOMKIS Analyse, Nr. 15, Leipzig

KRONE, ElisAbeth/ SCHeLler, HenRIK (2020): 10 J ahre KfW-Kommunalpanel: Methodik der Datenerhebung und -aufbereitung, Methodenpapier im Auftrag der KfW Bankengruppe, Berlin.

LenK, Thomas/ HesSe, Mario/ Starke, Tim (2019): Gemeindefinanzbericht Sachsen 2018/2019, in: Sachsenlandkurier, 6/ 2019, Dresden.

ManN, HenRy B., Whitney, Donald R. (1947): On a test of whether one of two random variables is stochastically larger than the other, in: The annals of mathematical statistics, Vol. 18, No. 1 , S. $50-60$

MengS, Christoph/ GÜnTHeR, NiKLAS (2018): Das Tal der Ahnungslosen 4. 0 - Der Breitbandausbau in Sachsen, KOMKIS Analyse, Nr. 11, Leipzig. 
MosSIG, IVo (2012): Stichproben, Stichprobenauswahlverfahren und Berechnung des minimal erforderlichen Stichprobenumfangs, in: Beiträge zur Wirtschaftsgeographie und Regionalentwicklung, No. 1-2012, Bremen, Inst. für Geographie, Bremen.

NEWSON, ROGER (2002): Parameters behind „nonparametric” statistics: Kendall's tau, Sommers' $\mathrm{D}$ and median differences, in: The Stata J ournal, Ausgabe 2, Nummer 1, S. 45-64.

Rasch, Bu ÖRn/ Friese, Malte/ Hofmann, Wilhelm/ Naumann, Ewald (2014): Quantitative Methoden 2: Einführung in die Statistik für Psychologen und Sozialwissenschaftler, 4. überarbeitete Aufl., Berlin, Heidelberg: Springer.

RotTmanN, Oliver/ HILBIG, CORINNA (2019): Studie zur Förderung des Breitband-Ausbaus im ländlichen Raum - Ergebnisse und Empfehlungen, Kompetenzzentrum Öffentliche Wirtschaft, Infrastruktur und Daseinsvorsorge e. V., PSPC Public Sector Project Consultans GmbH, Leipzig/ Berlin.

RotTmann, Oliver/ NeuHäUSER, KLAUS (2019): Tiefbau als strategische Option von Stadtwerken, in: MBI Energy Weekly/ Dow J ones, S. 9, 8.10.2019.

Rottmann, Oliver/ Mengs, Christoph/ Günther, NikLAs (2020): Smart City in Coronazeiten, in: Tagesspiegel Background Digitalisierung/KI, 18.5.2020, https:// background.tagesspiegel. de/ digitalisierung/ smart-city-in-corona-zeiten, abgerufen am 24.11.2020.

Sächsisches Gesetz über kommunale Zusammenarbeit (SächsKomZG) vom 15. April 2019.

SCHUHR, ROLAND (2017): Statistik und Wahrscheinlichkeitsrechnung. Eine Einführung für Wirtschaftswissenschaftler, Wirtschaftswissenschaftliche Fakultät der Universität Leipzig, Leipzig.

StATISTISCHE LANDESAMT DES FREISTAATES SACHSEN (2020): Fortschreibung des Bevölkerungsstandes (Basis 9.5.2011) zum 31.12.2018, Gemeinden (Gebietsstand ab 01.01.2019), GENESIS-Tabelle: 12410-020Z, Kamenz.

SteINER, ElisABETH/ BeNESCH, MiChAEL (2018): Der Fragebogen. Von der Forschungsidee zur SPSSAuswertung. 5. Auflage, utb Verlag, Wien.

SYDOW, J ULIA / HESSE, MARIO (2018): Kommunaler Investitionsbedarf im Freistaat Sachsen - Kommunalbefragung 2018, KOMKIS Analyse, Nr. 10, Leipzig.

SYDOW, J ULIA / HESSE, MARIO (2020): Kommunaler Investitionsbedarf im Freistaat Sachsen - Kommunal befragung 2020, KOMKIS Analyse, Nr. 17, Leipzig. 


\section{$5 \quad$ Anhang}

\section{$5.1 \quad$ Statistik}

\section{Untersuchungsobjekt und Untersuchungsmaterial}

Im Rahmen der Studie wurden alle sächsischen Kommunen mit eigenem Bau- bzw. Hauptamt befragt. Dies entspricht zum Gebietsstand 01.01.2018 einer Grundgesamtheit (N) von 322. Beauftragende Gemeinden sind nicht in der Stichprobe enthalten. Auch wurden Gemeinden, welche Teil eines Verwaltungsverbandes sind, nicht angeschrieben, da bereits der Verwaltungsverband in die Befragung einbezogen wurde. Eine räumliche Übersicht bietet Abbildung 28.

Abbildung 28: Verwaltungsgrenzen und -formen (Gebietsstand 01.01.2018)

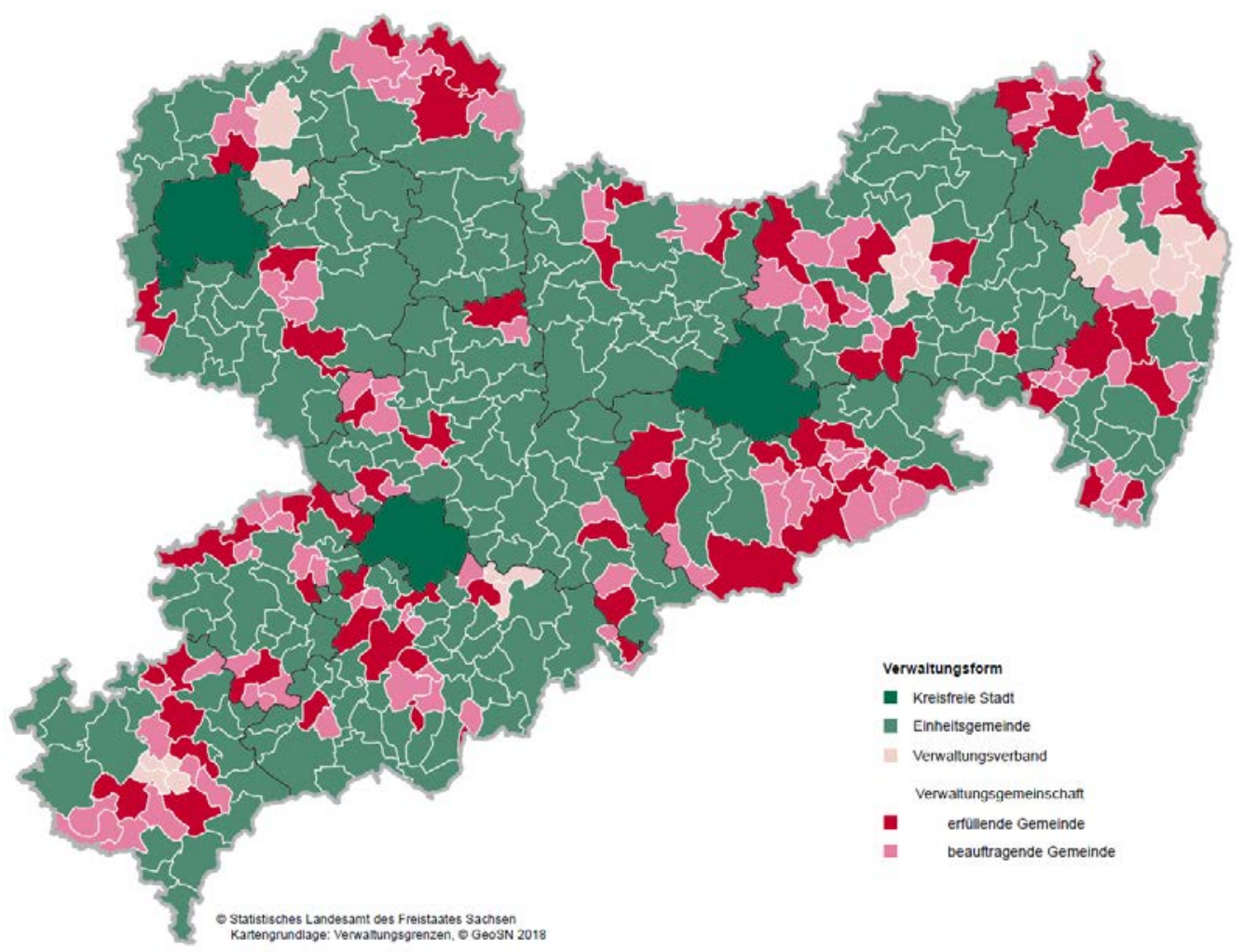

Quelle: Statistisches Landesamt des Freistaates Sachsen, 04.07.2019.

Die geplante Stichprobe erstreckte sich somit auf die genannten 322 Kommunen. Diese stützte sich auf zwei Befragungsmethoden: Erstens wurde der Fragebogen inklusive Anschreiben postalisch an alle kommunalen Bau- bzw. Hauptämter in Sachsen verschickt. ${ }^{48}$ Zweitens wurde eine Online-Befragung implementiert (LAMA POLL), wodurch den Kommunen die Form

48 Falls ein eigenständiges Bauamt existiert wurde dieses angeschrieben. Wenn dies nicht der Fall war, wurde das Hauptamt angeschrieben. 
der Beantwortung offen gelassen worden ist. ${ }^{49}$ Durch einen Zugangsschlüssel wurde technisch sichergestellt, dass alle Befragten nur einmal an der Befragung teilnehmen konnten. Die Befragung erfolgte anonym.

\section{Untersuchungsdurchführung und Datenaufbereitung}

Der Befragungszeitraum betrug acht Wochen. ${ }^{50}$ Die postalischen Rückläufe sowie die aus der Online-Befragung eingegangenen Antworten wurden dokumentiert und in einem ersten Arbeitsschritt in einen einheitlichen Datenpool zusammengeführt. In einem zweiten Schritt wurden die Rückläufe auf Vollständigkeit und Plausibilität ${ }^{51}$ überprüft. Danach wurden Absolutangaben (Euro bzw. Mio. Euro) in Pro-Kopf-Werte transformiert, um deren Vergleichbarkeit zu erhöhen. Im Weiteren wird auf den Fragebogenaufbau sowie die verwendeten Fragetypen und auf die daraus resultierenden Skalenniveaus eingegangen.

\section{Aufbau des Fragebogens und Auswahl der Fragetypen}

Der standardisierte Fragebogen besteht aus vier Teilen. In Teil A wurden allgemeine Angaben abgefragt, welche dazu dienen, die Merkmale der befragten Kommunen zu erfassen und so von der Stichprobe Rückschlüsse auf die zu untersuchende Grundgesamtheit treffen zu können. Teil B bildete den ersten Hauptteil der Befragung und bezog sich auf den kommunalen Straßenbau. Im zweiten Hauptteil - Teil C - wurden Fragen zum Breitbandausbau gestellt, die sich ebenfalls aus Einstellungs- und Verhaltensfragen zusammensetzten. Teil D schloss mit einer offenen Frage für weiterführende Anmerkungen ab.

\section{Skalenniveaus}

Auf Grund der gewählten Fragetypen enthielten die Antworten des Fragebogens unterschiedliche Skalenniveaus. Deren Eigenschaften werden nun vorgestellt, da sie die Anwendung statistischer Methoden einschränken. ${ }^{52}$

49 Ziel verschiedener Beantwortungsoptionen ist es, den zu Befragenden die Beantwortung möglichst einfach zu gestalten, wodurch eine höhere Rücklaufquote erreicht werden soll.

50 Auf Grund von zahlreichen Anfragen von sächsischen Kommunen, die ihre Teilnahme in Aussicht stellten, wurde der zunächst geplante Befragungszeitraum (04.09.2019 bis 02.10.2019) auf den 30.10.2019 verlängert.

51 Widersprüchliche Angaben in Teil A bzw. Antwortmuster in Teil B oder C führten zu einem Ausschluss aus der im Weiteren verwendeten Stichprobe. Zudem wurde eine Variable zur Art der Beantwortung hinzugefügt, um auf etwaige Verzerrungen durch die Erhebungsmethode zu rekurrieren.

52 Siehe hierzu insbesondere Steiner/ Benesch (2018). 
Die Nominalskala kann eine Unterscheidung zwischen Gleichheit und Ungleichheit treffen. Es handelt sich hierbei um einen qualitativen Vergleich, mit dessen Hilfe die Ausprägungen kontrastiert werden, jedoch besteht nicht die Möglichkeit, die einzelnen Merkmalsausprägungen in eine Reihenfolge zu bringen und so zu sortieren. ${ }^{53}$ Ordinalskalen zeichnen sich dadurch aus, dass eine Rangordnung zwischen den Antwortkategorien besteht. Die präzisen Abstände innerhalb der Rangordnung werden mit diesem Skalenniveau nicht erfasst, vielmehr wird davon ausgegangen, dass die Unterschiede zwischen den Optionen gleich groß sind. Dadurch können weiterführende statistische Analysemethoden, die genaue Abstandsinformationen benötigen, zum Teil nicht angewendet werden. Metrische Skalen haben den Vorteil, dass bei Nutzung einer Rangfolge die Abstände zwischen den einzelnen Rängen präzise interpretiert werden können. ${ }^{54}$

\section{Inferenzstatistik}

Die Übertragung von Befunden aus der Stichprobe auf die Grundgesamtheit erfolgt unter Anwendung von Methoden der inferenziellen Statistik ${ }^{55}$. Das gewählte Signifikanzniveau, demnach das Risiko eine Aussage anzunehmen, obwohl diese falsch ist, wird im weiteren Verlauf mit *** für das 1 Prozent Signifikanzniveau, das 5 Prozent Signifikanzniveau mit ** und das 10 Prozent Signifikanzniveau mit * angegeben.

\section{Hochrechnung und Gewichtung}

Um der sehr unterschiedlichen Struktur der sächsischen Kommunen Rechnung zu tragen, ${ }^{56}$ wurden sieben Einwohnerklassen gebildet. ${ }^{57}$ Anschließend wurden Antworten zu Aussagen

53 Eine Kopie des Fragebogens findet sich im Anhang dieser Studie.

54 Eine Unterscheidung hinsichtlich der Intervallskala und Verhältnisskala wird hier nicht vorgenommen. Es sei jedoch darauf hingewiesen, dass sich das metrische Skalenniveau aus den beiden genannten Skalen zusammensetzt.

55 Inferenzielle Statistik (synonym schließende Statistik) hat den Test sogenannter Hypothesen zum Gegenstand. Eine Hypothese bildet eine Annahme über die gesamte Grundgesamtheit, die mittels einer vorliegenden Stichprobe überprüft werden soll.

56 Die Struktur unterscheidet sich beispielsweise bezogen auf die Aufgabenzuteilung und den Auslagerungsgrad, der durchaus mit der Einwohnerzahl zusammenhängt. Vgl. Lenk/ Hesse/ Starke (2019), S. $94 \mathrm{f}$.

57 Die zehn Landkreise und die drei kreisfreien Städte bilden je eine eigene Gruppe. Die 322 kreisangehörigen Kommunen wurden in fünf Einwohnerklassen unterteilt: 161 Kommunen bilden die Gruppe unter 3000 Einwohner, 98 Kommunen bilden die Gruppe 3000 bis 5000 Einwohner, 92 Kommunen bilden die Gruppe 10000 bis 20000 Einwohner sowie 23 Kommunen bilden die Gruppe über 20000 Einwohner. 
gewichtet und numerische Angaben hochgerechnet, um Aussagen und Schätzungen über alle sächsischen Kommunen treffen zu können. ${ }^{58}$

Die Hochrechnung erfolgte unter der Annahme, dass die befragten Kommunen für ihre jeweilige Einwohnerklasse repräsentativ sind ${ }^{59}$ und der Mittelwert einer jeden Gruppe dem mittleren Wert in der Grundgesamtheit entspricht. Darauf basierend wurden Hochrechnungen für die vollzeitäquivalenten Stellen der Bau- bzw. Hauptämter, Kassenkredite, investive Schulden sowie die kommunalen Straßentypen erstellt. Beispielhaft wird nun die Hochrechnung der vollzeitäquivalenten Stellen dargestellt.

Für jede Einwohnerklasse g wird zunächst der Pro-Kopf-Mittelwert errechnet. Dieser bestimmt sich aus der Summe der vollzeitäquivalenten Stellen der befragten Kommunen (VZÄ) dividiert durch die Bevölkerung (BEV) der befragten Kommunen:

$$
\overline{V Z \ddot{A}_{g}}=\frac{\sum_{i=1}^{j} V Z \ddot{A}_{g}}{\sum_{i=1}^{j} B E V_{g}}
$$

Anschließend wird die Hochrechnung für Sachsen erstellt, wobei der Pro-Kopf-Mittelwert der Einwohnerklasse g mit der jeweiligen Einwohnerzahl der amtlichen Statistik ${ }^{60}$ multipliziert wird:

$$
V Z \ddot{\mathrm{A}}_{\text {Sachsen }}=\sum_{g=1}^{n} * \overline{V Z \ddot{\mathrm{A}}_{g}} * B e v_{g}
$$

Nun wird die Gewichtung als Methode erläutert. Da die Rücklaufquote zwischen den sieben Einwohnerklassen variiert, ist eine Gewichtung der Aussagen notwendig, um Aussagen über die Grundgesamtheit aller sächsischen Kommunen treffen zu können. Es wird in einem ersten Schritt für jede Gruppe $g$ ein Gewichtungsfaktor $w$ bestimmt. Dieser bildet sich aus dem Anteil der Kommunen einer Gruppe zur Grundgesamtheit ( $N_{-} G_{g} / N_{-} G_{\text {gesamt }}$ ) im Verhältnis zum Anteil der befragten Kommunen zur Gesamtzahl der befragten Kommunen (N_BKg/ N_BKgesamt):

$$
W_{g}=\frac{\frac{N_{-} G G_{g}}{N_{-} G G_{\text {gesamt }}}}{\frac{N_{-} B K g}{N_{-} B K_{\text {gesamt }}}}
$$

In einem zweiten Schritt werden die Ausprägungen mit dem jeweiligen Gewichtungsfaktor aus (3) multipliziert, wodurch sich ein neues Verhältnis zwischen den Antwortkategorien

58 Das Verfahren zur Gewichtung und Hochrechnung entspricht der Methodik des KfW-Kommunalpanels. Vgl. Krone/ Scheller (2020), S. 11-16.

59 Mit dem Rücklauf von 73 Kommunen können Aussagen auf dem 10\%Niveau getroffen werden. Vgl. Mossig (2012), S. 21.

60 Es wird die Bevölkerungsfortschreibung zum 31.12. 2018 verwendet. Siehe Statistisches Landesamt des Freistaates Sachsen (2020). 
bildet. Trotz der hohen Rücklaufquote existieren nicht in allen Fragen für die sieben Einwohnerklassen Ausprägungen. Falls das Fehlen von Ausprägungen kausal ist, ${ }^{61}$ wird die Methodik zur Hochrechnung bzw. Gewichtung modifiziert, so dass eine geringere Anzahl an Gruppen in diesem Fall angenommen wird. Falls es keinen kausalen Grund gibt, bleibt die Anzahl der Gruppen aufrechterhalten. Wenn nicht in allen Gruppen Ausprägungen existieren, wurde dies im Weiteren vermerkt. Neben der Einwohnergewichtung wurde zudem eine Gewichtung nach dem Zersiedlungsindex und der Siedlungs- und Verkehrsfläche vorgenommen. Der Zersiedlungsindex ist ein Indikator aus drei Teilkomponenten, wobei gilt, dass eine Landschaft umso stärker zersiedelt ist, je mehr Fläche bebaut ist und je geringer die Ausnutzung der gestreuten Siedlungsfläche für Wohn- und Arbeitsfläche ist. ${ }^{62}$ Die jeweiligen Gewichtungsmaßstäbe werden immer dann in der Studie vorgestellt, wenn es signifikante Veränderungen hin zur ungewichteten Variante gibt, da andernfalls der Erklärungsgehalt der zusätzlichen Gewichtung begrenzt ist.

\section{Rangkorrelationskoeffizienten}

Liegen keine metrisch skalierten Merkmalsträger vor, so ist die Berechnung von Zusammenhangsmaßen nur eingeschränkt möglich. Alternativ lassen sich für ordinal skalierte Merkmalsträger zwei unterschiedliche Korrelationsmaße berechnen: der Rangkorrelationskoeffizient nach SPEARMAN und KendalLSTAU-B. Die Idee hinter beiden Maßen liegt darin, dass sich sowohl ordinal als auch metrisch skalierte Merkmalsträger in eine Reihenfolge ordnen lassen. Werden zwei Datenreihen untersucht, so kann eine Datenreihe geordnet werden und anschließend geprüft werden, inwiefern sich die zweite Datenreihe mitgeordnet hat.

Der Rangkorrelationskoeffizient nach Spearman $\left(\boldsymbol{r}_{\boldsymbol{g}}\right)$ ist eine Erweiterung des Korrelationskoeffizienten nach BravaIS-PEARSON und ist auf ordinale Daten spezialisiert. ${ }^{63}$ Auch hier wird der Zusammenhang zwischen zwei Variablen gemessen. Der Koeffizient berechnet sich wie folgt ${ }^{64}$ :

$$
r_{g}=\frac{\operatorname{cov}(r g(x), r g(y))}{s_{r g(x)^{*}} \operatorname{rg}(y)}
$$

Die Kovarianz zwischen zwei Variablen wird durch das Produkt der Standardabweichungen beider Merkmalsausprägungen dividiert. Die Interpretation dieses Maßes ist gleich des Rangkorrelationskoeffizienten nach BRAVAIS-PEARSON. Der Wert von SPEARMAN $r_{g}$ liegt stets zwischen

\footnotetext{
61 Beispiel ist in Frage A5 das Fehlen von Angaben zu Kreisstraßen für kreisangehörige Kommunen kausal, da diese keine Kreisstraßen in ihrem kommunalen Eigentum aufweisen.

62 Vgl. IÖR-Monitor (2020).

63 Vgl. Schuhr (2017), S. 109.

64 Vgl. ebd., S. 110.
} 
-1 und $+1 .{ }^{65}$ Ein Wert von +1 gibt einen gleichsinnigen perfekten monotonen Zusammenhang an, während ein Wert von 0 keinerlei Korrelation wiederspiegelt. Im Falle eines Wertes von -1 liegt ein perfekter gegensinniger monotoner Zusammenhang vor. Im Intervall zwischen -1 und +1 ergeben sich partielle Korrelationen.

Vergleichbar mit dem Rangkorrelationskoeffizient nach SPEARMAN untersucht KENDALLS TAU-B $(\boldsymbol{\tau})$ ebenfalls ordinal skalierte Merkmalsträger auf potenzielle Korrelationen. Zunächst werden Ränge beider Variablenreihen gebildet. Wie oben beschrieben werden die Daten anschließend nach den Rängen einer der beiden Datenreihen geordnet und überprüft. Die Überprüfung erfolgt anhand des paarweisen Vergleiches der Ränge der zweiten Datenreihe, wobei hier zwischen konkordanten und diskordanten Paaren unterschieden wird. ${ }^{66}$ Der Vorteil von Kendalls Tau liegt darin, dass dieser bei einer kleinen Stichprobengröße auf Ausreißer robust reagiert. ${ }^{67}$ Dies kann von Vorteil sein, sofern die Grundgesamtheit 20 Ausprägungen nicht übersteigt. ${ }^{68}$ In der vorliegenden Studie wird dieser neuralgische Wert zwar überschritten, j edoch sind Ausreißer vorhanden. Dementsprechend werden beide Korrelationsmaße berechnet und ausgewertet. KENDALLS TAU-B berechnet sich wie folgt ${ }^{69}$ :

$$
\tau=\frac{S}{\sqrt{\frac{N(N-1)}{2}-T_{x} * \sqrt{\frac{N(N-1)}{2}-T_{y}}}}
$$

S beschreibt die Differenz aus dem Auftreten zwischen Proversionen und Inversionen. N beschreibt die Anzahl der Paare, wohingegen $T_{x}$ und $T_{y}$ die Anzahl jener Paare beschreibt, bei denen der erste Wert der Paarung gleich dem zweiten Wert ist. ${ }^{70}$ Kongruent, wie beim Rangkorrelationskoeffizient nach Spearman, ergibt sich ebenfalls ein mögliches Analyseintervall von -1 bis +1 .

\section{Hypothesentests}

Neben der Hochrechnung, der Gewichtung und den Rangkorrelationskoeffizienten werden überdies Hypothesentests zur Auswertung der Umfrage verwendet. Hierbei wird der MANN-

65 Vgl. ebd., S. 111.

66 Bei konkordanten Paaren wird die natürliche Reihenfolge der Ränge eingehalten, während dies bei diskordanten Paaren nicht der Fall ist. Vgl. Newson (2002), S. 46.

67 Vgl. ebd., S. 50.

68 Vgl. Ebermann (2010).

69 Die Formel (5) beschreibt Kendalls TAU-B. Generell existieren drei verschiedene Varianten. Üblicherweise werden jedoch nur zwei Varianten, TAU-B und TAU-C verwendet. Der Unterschied zwischen beiden Varianten liegt darin, wie die verbundenen Ränge miteinander behandelt werden. Generell ähneln sich jedoch beide Varianten. Vgl. Ebermann (2010); Newson (2002), S. 46.

70 Vgl. ebd., S. $46 \mathrm{f}$. 
WHITNEY-U-TEST angewendet. Dieses Verfahren ${ }^{71}$ untersucht, ob die Angaben zweier unabhängiger Gruppen zu einem Merkmal zufälligen oder systematischen Einflüssen unterliegen. Der Test findet bei ordinalskalierten Daten Anwendung. Dazu werden den Datenpunkten Ränge zugeordnet und entsprechend der Ränge sortiert. Durch den U-Test wird nun die Nullhypothese geprüft, ob sich die beiden Gruppen hinsichtlich ihrer Angaben zu dem Merkmal gleichen. ${ }^{72}$

Zunächst werden die Ränge der jeweiligen Gruppen summiert und anschließend durch die Anzahl der Teilnehmenden in Gruppe i geteilt, um die mittlere Rangsumme bzw. den Mittleren Rang zu erhalten, der für die Interpretation des U-Tests bedeutend ist ${ }^{73}$ :

$$
\overline{R_{l}}=\frac{T_{i}}{n_{i}}
$$

Dabei entspricht $\bar{R}_{\iota}$ dem mittleren Rang der Gruppe i, $T_{i}$ der Summe der Rangplätze in Gruppe i und $n_{i}$ der Anzahl der Teilnehmenden in Gruppe i. Bestehen Unterschiede in den mittleren Rängen der beiden Gruppen, muss überprüft werden, ob diese statistisch signifikant sind. Ist der Unterschied nicht statistisch signifikant, so kann auf Basis der Stichprobe nicht die Schlussfolgerung abgeleitet werden, dass sich das betrachtete Merkmal in der Grundgesamtheit unterscheidet. Dazu wird zunächst die Rangplatzüberschreitung U berechnet. Diese wird gebildet, indem für jeden Rangplatz eines Teilnehmenden der Gruppe i die Anzahl der Teilnehmenden aus der anderen Gruppe gezählt wird, die sich über diesem Rangplatz befinden: ${ }^{74}$

$$
U=n_{1} * n_{2}+\frac{n_{1} *\left(n_{1}+1\right)}{2}-T_{1}
$$

Dabei entspricht $U$ dem U-Wert, $n_{1}$ der Anzahl der Teilnehmenden der Gruppe $1, n_{2}$ der Anzahl der Teilnehmenden der Gruppe 2 und $T_{1}$ der Rangsumme der Gruppe 1. Analog wird die Rangplatzüberschreitung U' für die zweite Gruppe berechnet ${ }^{75}$ :

$$
U^{\prime}=n_{1} * n_{2}+\frac{n_{2} *\left(n_{2}+1\right)}{2}-T_{2}
$$

Nach der Nullhypothese des U-Tests muss $U=U^{\prime}$ gelten. Der Signifikanzwert wird nach folgender Formel (9) berechnet $^{76}$ :

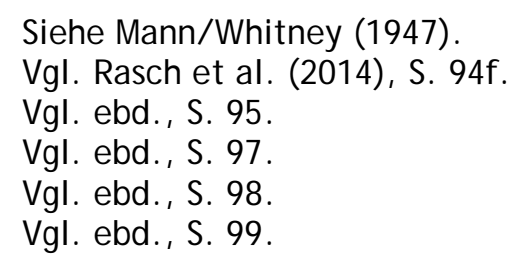




$$
z_{U}=\frac{U-\mu_{U}}{\sigma_{U}}
$$

In der Formel (10) stellt $\mu_{U}$ den erwarteten Mittelwert und die Formel (11) $\sigma_{U}$ die Streuung von $U$ dar. Beide werden wie folgt ermittelt ${ }^{77}$ :

$$
\begin{aligned}
& \mu_{U}=\frac{n_{1} * n_{2}}{2} \\
& \sigma_{U}=\sqrt{\frac{n_{1} * n_{2} *\left(n_{1}+n_{2}+1\right)}{12}}
\end{aligned}
$$

Falls eine der Strichproben $n_{1}$ oder $n_{2}$ größer 20 ist nähert sich die Verteilung einer Normalverteilung an. Folglich wird der ermittelte Wert für $z_{U}$ mit Werten der asymptotischen Signifikanz abgeglichen. Stichproben, die kleiner gleich 20 sind, werden mithilfe der exakten Signifikanz überprüft. ${ }^{78}$ Die im Weiteren durchgeführten Hypothesentests erfolgen immer mit mindestens einer Stichprobe, die größer 20 ist, wodurch als Prüfgröße immer die asymptotische Signifikanz verwendet wird. 


\subsection{Fragbogen}

\section{A Allgemeine Angaben}

Die folgenden allgemeinen Angaben ermöglichen uns die Vergleichbarkeit der Resultate. Selbstverständlich werden alle Angaben anonymisiert ausgewertet und vertraulich behandelt.

\section{A. 0 Bitte geben Sie die Stammdaten Ihrer Kommune ein.}

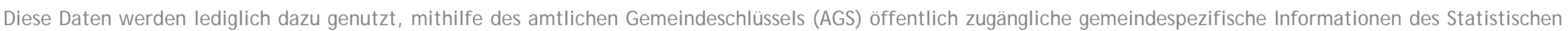
Landesamtes des Freistaates Sachsen abzurufen. Ein weiterer Abgleich mit Daten geschieht nicht.

Sofern Sie den AGS nicht angeben möchten, bitten wir Sie, mit den Fragen A. 0.1 bis A. 0.4 fortzufahren.

Name der Kommune:

amtlicher Gemeindeschlüssel: [weiter mit A.1]

A.0.1 Welcher Art ist Ihre Kommune zuzuordnen?

$\square$ kreisangehörige Stadt oder Gemeinde

$\square$ Landkreis

$\square$ kreisfreie Stadt

A.0.2 Wie viele Einwohner und Wohngebäude zählt Ihre Kommune? (Stand 31.12.2018)

Einwohner

Wohngebäude

A.0.3 Wie hoch ist Ihr kommunaler Schuldenstand, unterteilt in Kassenkredite und fundierte/ investive Schulden? (Stand 31.12.2018)

Kassenkredite: $€$

fundierte/ investive Schulden: $€$ 
A.0.4 Welche Fläche umfasst Ihre Kommune insgesamt? Wie verteilt sich die Fläche auf Wohnbau- und Verkehrsfläche? (Stand 31.12.2018) ha Gesamtfläche ha Wohnbaufläche ha Verkehrsfläche

Falls Ihre Kommune eine kreisfreie Stadt oder ein Landkreis ist, überspringen Sie bitte Frage A.1 und fahren Sie mit A.2 fort.

A.1 Ist Ihre Kommune Mitglied eines Verwaltungsverbandes oder Teil einer Verwaltungsgemeinschaft?

A. 2 Besitzt Ihre Kommune ein Bauamt?

$\square$ ja [weiter mit Frage A.2.1]

$\square$ nein [weiter mit Frage A.2.2]

A.2.1 Wenn ja: Wie viele vollzeitäquivalente Stellen (VZÄ) besitzt Ihr Bauamt? (Stand 31.12.2018) VZÄ

A.2.2 Wenn nein: Wie viele vollzeitäquivalente Stellen (VZÄ) besitzt Ihr Hauptamt? (Stand 31.12.2018)

VZÄ 
Kommunen unterhalten einen Großteil der öffentlichen Straßen. Neben der Klasse der Gemeindestraßen unterscheiden sich die höherrangigen Kreisstraßen sowie Staatsstraßen (Landesstraßen).

A.3 Wie viele Kilometer Straße (Staat, Gemeinde, Kreis) durchlaufen Ihre Kommune insgesamt? (Stand 31.12.2018)

Bundes- und Landesstraßen: $\mathrm{km}$

Kreisstraßen: $\mathrm{km}$

Gemeindestraßen: $\mathrm{km}$

A.4 Wie hoch war der Anteil der leitungsgebundenen Breitbandverfügbarkeit der privaten Haushalte von 50 Mbit/s in Ihrer Kommune? (Stand 31.12.2018)

Wenn Ihnen die Information nicht vorliegt, kann diese auf der Webseite des Bundesministeriums für Verkehr und digitale Infrastruktur (BMVI) im Breitbandatlas abgerufen werden.

$\%$

A.5 Erfassen Sie die Breitbandverfügbarkeit der Gewerbegebiete in Ihrer Kommune?
$\square$ ja [weiter mit Frage A.6]
$\square$ nein $\quad$ [weiter mit Teil B]

A.6 Wenn ja: Wie viel Prozent der gemeldeten Gewerbe hatte eine Breitbandverfügbarkeit von mindestens 50 Mbit/ s? (Stand: 31.12. 2018) 


\section{B Tiefbaukoordinierung}

In diesem Teil werden das Verhalten sowie Meinungen bzw. Positionen Ihrer Kommune abgefragt.

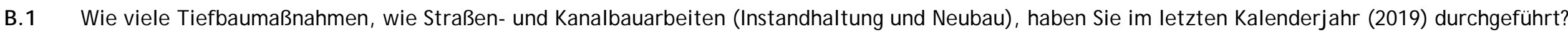

Anzahl Instandhaltung:

Anzahl Neubau:

$\square$ weiß nicht

B.2 Führen Sie verschiedene Baumaßnahmen im Tiefbau innerhalb Ihrer Kommune oder im Rahmen einer interkommunalen Kooperation gebündelt durch?
$\square$ immer gebün-
$\square$ oft gebündelt
$\square$ gelegentlich ge- bündelt
selten gebündelt
$\square$ nie gebündelt
$\square$ weiß delt gebuindelt

B.3 Stimmen Sie aus Sicht Ihrer Kommune zu, dass die folgenden Hindernisse im der Rahmen Koordinierung von Tiefbaumaßnahmen bestehen?

\begin{tabular}{|c|c|c|c|c|c|}
\hline & $\begin{array}{c}\text { stimme } \\
\text { gar } \\
\text { nicht zu }\end{array}$ & $\begin{array}{c}\text { stimme e- } \\
\text { her nicht } \\
\text { zu }\end{array}$ & neutral & $\begin{array}{l}\text { stimme e- } \\
\text { her zu }\end{array}$ & $\begin{array}{l}\text { stimme } \\
\text { voll zu }\end{array}$ \\
\hline unzureichende Personalausstattung & $\square$ & $\square$ & $\square$ & $\square$ & $\square$ \\
\hline rechtliche Auflagen (Vergaberecht) & $\square$ & $\square$ & $\square$ & $\square$ & $\square$ \\
\hline unklare Zuständigkeiten bei übergeordneten Einheiten & $\square$ & $\square$ & $\square$ & $\square$ & $\square$ \\
\hline Informations- und Kommunikationsprobleme & $\square$ & $\square$ & $\square$ & $\square$ & $\square$ \\
\hline Widerstände übergeordneter öffentlicher Ebenen & $\square$ & $\square$ & $\square$ & $\square$ & $\square$ \\
\hline Vereinbarungskosten (Verhandlungen, Verträge etc.) & $\square$ & $\square$ & $\square$ & $\square$ & $\square$ \\
\hline lokale Egoismen (Kirchturmdenken) & $\square$ & $\square$ & $\square$ & $\square$ & $\square$ \\
\hline Sonstige: & $\square$ & $\square$ & $\square$ & $\square$ & $\square$ \\
\hline
\end{tabular}

\begin{tabular}{|c|}
\hline $\begin{array}{c}\text { weiß } \\
\text { nicht }\end{array}$ \\
\hline$\square$ \\
\hline$\square$ \\
\hline$\square$ \\
\hline$\square$ \\
\hline$\square$ \\
\hline$\square$ \\
\hline$\square$
\end{tabular}


B.4 Wie schätzen Sie die Instrumente zur Förderung der Koordinierung im kommunalen Tiefbau hinsichtlich ihrer Effektivität ein?

\begin{tabular}{|l|c|c|c|c|c|}
\cline { 3 - 5 } \multicolumn{1}{l|}{} & $\begin{array}{c}\text { nicht ef- } \\
\text { fektiv }\end{array}$ & $\begin{array}{c}\text { eher } \\
\text { nicht ef- } \\
\text { fektiv }\end{array}$ & neutral & $\begin{array}{c}\text { eher ef- } \\
\text { fektiv }\end{array}$ & $\begin{array}{c}\text { sehr ef- } \\
\text { fektiv }\end{array}$ \\
\hline Leitfaden / Handlungsempfehlung zur Baukoordinierung & $\square$ & $\square$ & $\square$ & $\square$ & $\square$ \\
\hline vereinfachtes Genehmigungsverfahren & $\square$ & $\square$ & $\square$ & $\square$ & $\square$ \\
\hline höherer Fördersatz & $\square$ & $\square$ & $\square$ & $\square$ & $\square$ \\
\hline höherer Förderanteil & $\square$ & $\square$ & $\square$ & $\square$ & $\square$ \\
\hline Weiterbildungsmaßnahmen & $\square$ & $\square$ & $\square$ & $\square$ \\
\hline Sonstige: & $\square$ & $\square$ & $\square$ & $\square$ & $\square$ \\
\hline
\end{tabular}

\begin{tabular}{|c|}
\hline $\begin{array}{c}\text { weiß } \\
\text { nicht }\end{array}$ \\
\hline$\square$ \\
\hline$\square$ \\
\hline$\square$ \\
\hline$\square$ \\
\hline$\square$ \\
\hline$\square$ \\
\hline
\end{tabular}

B.5 Gibt es eine Dokumentation über vorhandene Leitungen oder andere Infrastrukturen in Ihrer Kommune? (Mehrfachnennung möglich)

\section{$\square$ Register}

[weiter mit B.6] $\square$ zentrales Kataster

[weiter mit B.6] $\square$

[weiter mit B.6]
Sonstige:

$\square$ weiß nicht 
Wie ist der Zugang zu den Informationen für Dritte (z.B. Bürger, Unternehmen, etc.) zurzeit in Ihrer Kommune gestaltet?

$\square$ nur intern

$\square$ auf Anfrage gegen Gebühr

$\square$ auf Anfrage kostenfrei

$\square$ frei zugänglich

$\square$ Sonstige:

$\square$ weiß nicht

B.7 Wie schätzen Sie die Bedeutung der Speicherung folgender Informationen hinsichtlich ihrer Wichtigkeit für eine erfolgreiche Koordinierung von kommunalen Tiefbaumaßnahmen unabhängig Ihrer Antwort zu der Frage B5 ein?

Bitte geben Sie an, wie wichtig Ihnen die gespeicherten Informationen für eine erfolgreiche Koordinierung von kommunalen Tiefbaumaßnahmen erscheinen.

\begin{tabular}{|c|c|c|c|c|c|}
\hline & $\begin{array}{l}\text { völlig un- } \\
\text { wichtig }\end{array}$ & $\begin{array}{l}\text { eher un- } \\
\text { wichtig }\end{array}$ & neutral & $\begin{array}{l}\text { eher wich- } \\
\text { tig }\end{array}$ & $\begin{array}{l}\text { sehr wich- } \\
\text { tig }\end{array}$ \\
\hline \begin{tabular}{|l} 
Baujahr \\
J ahr der Fertigstellung
\end{tabular} & $\square$ & $\square$ & $\square$ & $\square$ & $\square$ \\
\hline $\begin{array}{l}\text { räumliche Lage } \\
\text { Geodaten etc. }\end{array}$ & $\square$ & $\square$ & $\square$ & $\square$ & $\square$ \\
\hline $\begin{array}{l}\text { Art der Infrastruktur } \\
\text { Straße, Kanal etc. }\end{array}$ & $\square$ & $\square$ & $\square$ & $\square$ & $\square$ \\
\hline $\begin{array}{l}\text { Bauweise } \\
\text { Informationen zur Bautechnik }\end{array}$ & $\square$ & $\square$ & $\square$ & $\square$ & $\square$ \\
\hline $\begin{array}{l}\text { verbaute Breitbandtechnologie } \\
\text { Informationen zur verbauten Breitbandtechnologie (FTTC, FFTH, ...) }\end{array}$ & $\square$ & $\square$ & $\square$ & $\square$ & $\square$ \\
\hline $\begin{array}{l}\text { Eigentümer } \\
\text { Information zu Eigentümer }\end{array}$ & $\square$ & $\square$ & $\square$ & $\square$ & $\square$ \\
\hline Sonstige: & $\square$ & $\square$ & $\square$ & $\square$ & $\square$ \\
\hline
\end{tabular}

\begin{tabular}{|c|}
\hline weiß nicht \\
\hline$\square$ \\
\hline$\square$ \\
\hline$\square$ \\
\hline$\square$ \\
\hline$\square$ \\
\hline$\square$ \\
\hline
\end{tabular}




\section{Breitband}

An dieser Stelle folgen Fragen zu der Umsetzung des öffentlich geförderten Breitbandausbaus.

\section{C.1 Wie bewerten Sie die nachfolgenden Faktoren für den lokalen Breitbandausbau bezogen auf Ihre Kommune?}

Bitte erstellen sie eine Rangfolge, indem Sie die Zahlen von „1“ bis „6“ eintragen. Dabei stellt „1“ den höchsten und "6" den niedrigsten Rang dar (Schulnoten).

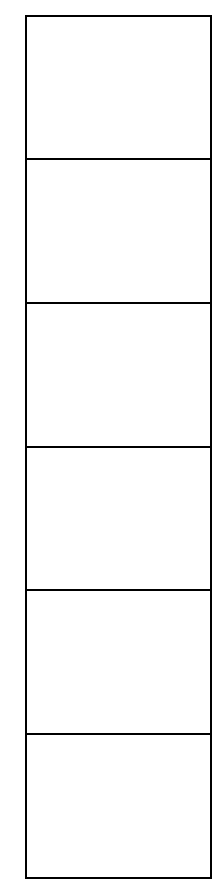

vorgegebene Ausbauziele des Landes oder des Bundes

Nachfrage der privaten Haushalte vor Ort

Nachfrage der bestehenden Unternehmen

$\square$ weiß nicht

Bremsen des demographischen Wandels

Förderung von Unternehmensansiedlung

Sonstige: 
C.2 Für welche Bereiche schätzen Sie die Bedeutung des Breitbandausbaus aus Sicht Ihrer Kommune als besonders hoch ein? Sie können bis zu drei Felder ankreuzen.

Hinweis: Sofern Sie mehr als drei Felder ankreuzen, werden die ersten drei gewertet.
$\square$ Privathaushalte
$\square$ Gewerbe und Dienst
$\square$ Landwirtschaft leistungen
$\square$ Digitalisierung der Verwaltung
$\square$ Verkehr und Mobili-

$\square$ Gesundheitssektor

$\square$ Energiesektor

tät

$\square$ Sonstige:

$\square$ weiß nicht

Bei dem sogenannten Betreibermodell erfolgt die Errichtung und Bereitstellung der passiven Infrastruktur durch die Kommune während ein Telekommunikationsunternehmen die aktive Infrastruktur errichtet und Internetdienste für Dritte anbietet. Alternativ ist die Ausschreibung und vollständige Weitergabe der Fördermittel an einen privaten Anbieter im Rahmen des sogenannten Wirtschaftlichkeitslückenmodells möglich.

C.3 Haben Sie als Kommune oder eines Ihrer kommunalen Unternehmen bereits ein oder mehrere Projekte zum Breitbandausbau durchgeführt (Betreibermodell) oder begleitet (Wirtschaftlichkeitslückenmodell)?
$\square$ ja
$\square$ nein
$\square$ weiß nicht 


\section{C.4 Für wie wichtig schätzen Sie aus Sicht Ihrer Kommune die folgenden Hemmnisse des öffentlich geförderten Breitbandausbaus ein?}

\begin{tabular}{|c|c|c|c|c|c|}
\hline & $\begin{array}{l}\text { völlig un- } \\
\text { wichtig }\end{array}$ & $\begin{array}{l}\text { eher un- } \\
\text { wichtig }\end{array}$ & neutral & $\begin{array}{l}\text { eher } \\
\text { wichtig }\end{array}$ & $\begin{array}{l}\text { sehr wich- } \\
\text { tig }\end{array}$ \\
\hline $\begin{array}{l}\text { Vergabeverhalten } \\
\text { (z.B. Diskontinuität von Ausschreibungen) }\end{array}$ & $\square$ & $\square$ & $\square$ & $\square$ & $\square$ \\
\hline Bürokratie & $\square$ & $\square$ & $\square$ & $\square$ & $\square$ \\
\hline Pflicht zur europaweiten Ausschreibung & $\square$ & $\square$ & $\square$ & $\square$ & $\square$ \\
\hline Personalprobleme & $\square$ & $\square$ & $\square$ & $\square$ & $\square$ \\
\hline Planungsfehler & $\square$ & $\square$ & $\square$ & $\square$ & $\square$ \\
\hline große Entfernung zwischen einzelnen Ortslagen & $\square$ & $\square$ & $\square$ & $\square$ & $\square$ \\
\hline fehlende Tiefbaukapazitäten & $\square$ & $\square$ & $\square$ & $\square$ & $\square$ \\
\hline keine Dringlichkeit & $\square$ & $\square$ & $\square$ & $\square$ & $\square$ \\
\hline Sonstige: & $\square$ & $\square$ & $\square$ & $\square$ & $\square$ \\
\hline
\end{tabular}

\begin{tabular}{|c|}
\hline $\begin{array}{c}\text { weiß } \\
\text { nicht }\end{array}$ \\
\hline$\square$ \\
\hline$\square$ \\
\hline$\square$ \\
\hline$\square$ \\
\hline$\square$ \\
\hline$\square$ \\
\hline$\square$ \\
\hline$\square$ \\
\hline
\end{tabular}


C.5 Stimmen Sie aus Sicht Ihrer Kommune zu, dass die folgenden Hindernisse im Rahmen der Fördermittelprogrammatik, beispielsweise der Digitale Offensive Sachsen, bestehen?

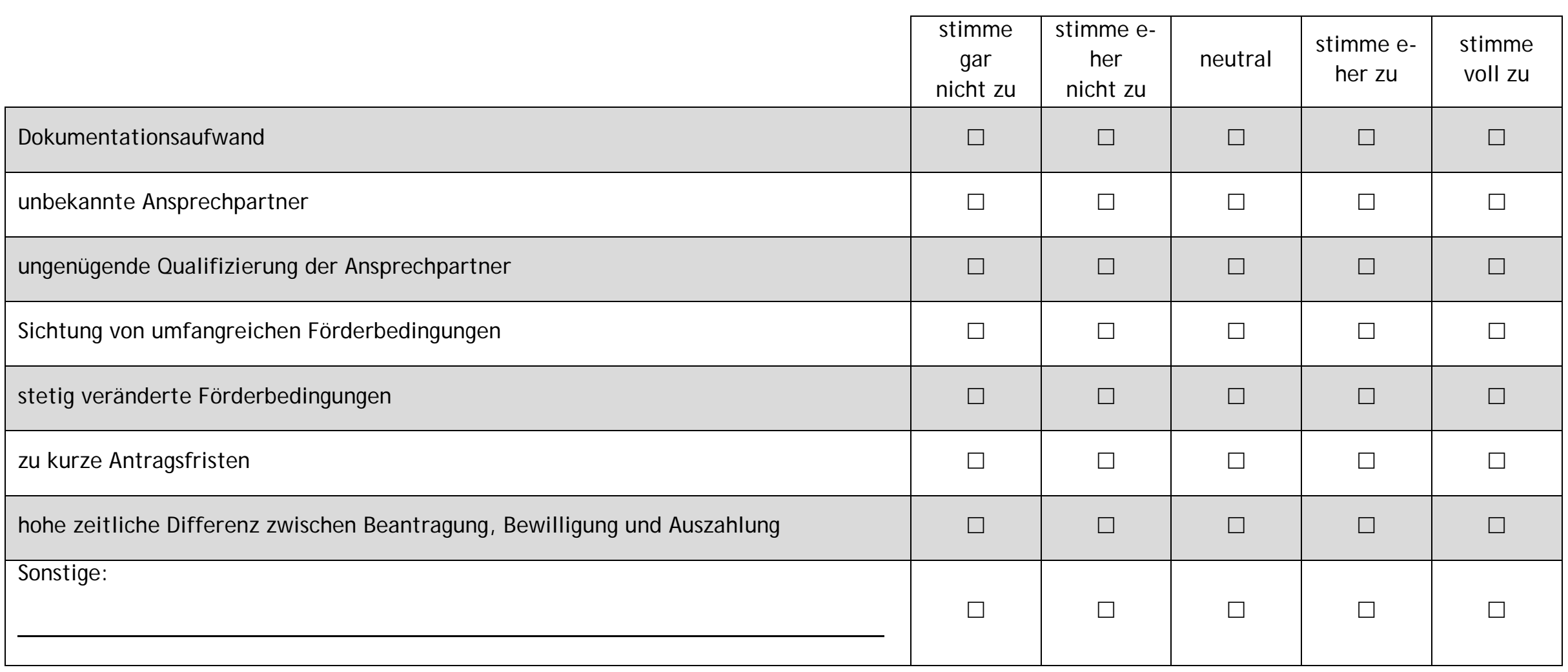

\begin{tabular}{|c|}
\hline $\begin{array}{c}\text { weiß } \\
\text { nicht }\end{array}$ \\
\hline$\square$ \\
\hline$\square$ \\
\hline$\square$ \\
\hline$\square$ \\
\hline$\square$ \\
\hline$\square$ \\
\hline$\square$ \\
\hline
\end{tabular}


C.6 Wie schätzen Sie Instrumente zur Förderung des Breitbandausbaus hinsichtlich ihrer Effektivität ein? Welche Verwaltungsebene sollte diese Maßnahme aus Sicht Ihrer Kommune hauptsächlich durchführen?

Die Zuständigkeit fragt ab, welche Verwaltungsebene für die Fördermaßnahme zuständig sein sollte.

\begin{tabular}{|c|c|c|c|c|c|c|}
\hline & \multicolumn{6}{|c|}{ Effektivität } \\
\hline & 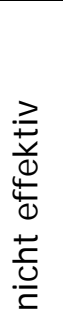 & 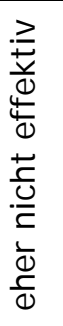 & $\frac{\frac{n}{\bar{\Phi}}}{\frac{\omega}{\bar{\Phi}}}$ & 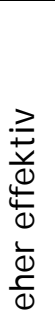 & 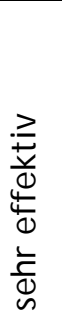 & 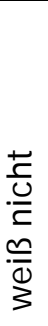 \\
\hline Förderung der interkommunalen Kooperation (Zweckvereinbarung etc.) & $\square$ & $\square$ & $\square$ & $\square$ & $\square$ & $\square$ \\
\hline vollständige Übertragung der Aufgabe auf kommunale Unternehmen & $\square$ & $\square$ & $\square$ & $\square$ & $\square$ & $\square$ \\
\hline Qualifizierungs- und Weiterbildungsmaßnahmen & $\square$ & $\square$ & $\square$ & $\square$ & $\square$ & $\square$ \\
\hline externe Beratung & $\square$ & $\square$ & $\square$ & $\square$ & $\square$ & $\square$ \\
\hline Expertenworkshops & $\square$ & $\square$ & $\square$ & $\square$ & $\square$ & $\square$ \\
\hline Nachfrageförderung durch Gutscheine für Haushaltsanschlüsse (Voucher) & $\square$ & $\square$ & $\square$ & $\square$ & $\square$ & $\square$ \\
\hline Sonstige: & $\square$ & $\square$ & $\square$ & $\square$ & $\square$ & $\square$ \\
\hline
\end{tabular}

\begin{tabular}{|c|c|c|c|c|}
\hline \multicolumn{5}{|c|}{ Zuständigkeit } \\
\hline 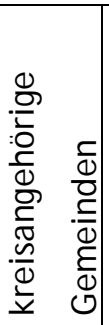 & 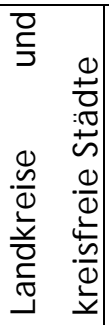 & 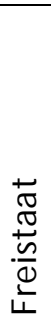 & 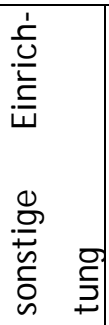 & 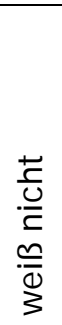 \\
\hline$\square$ & $\square$ & $\square$ & $\square$ & $\square$ \\
\hline$\square$ & $\square$ & $\square$ & $\square$ & $\square$ \\
\hline$\square$ & $\square$ & $\square$ & $\square$ & $\square$ \\
\hline$\square$ & $\square$ & $\square$ & $\square$ & $\square$ \\
\hline$\square$ & $\square$ & $\square$ & $\square$ & $\square$ \\
\hline$\square$ & $\square$ & $\square$ & $\square$ & $\square$ \\
\hline$\square$ & $\square$ & $\square$ & $\square$ & $\square$ \\
\hline
\end{tabular}


C.7 Für wie hilfreich schätzen Sie die Umsetzungshilfen des Gesetzes zur Erleichterung des Ausbaus digitaler Hochgeschwindigkeitsnetze (DigiNetzG) ein?

\begin{tabular}{|c|c|c|c|c|c|c|}
\hline & $\begin{array}{l}\text { nicht hilf- } \\
\text { reich }\end{array}$ & $\begin{array}{l}\text { eher nicht } \\
\text { hilfreich }\end{array}$ & $\begin{array}{l}\text { teils / } \\
\text { teils }\end{array}$ & $\begin{array}{l}\text { eher hilf- } \\
\text { reich }\end{array}$ & $\begin{array}{l}\text { sehr hilf- } \\
\text { reich }\end{array}$ & $\begin{array}{l}\text { weiß } \\
\text { nicht }\end{array}$ \\
\hline Nationale Streitbeilegungsstelle & $\square$ & $\square$ & $\square$ & $\square$ & $\square$ & $\square$ \\
\hline online GIS-Tool „ISA-Planung“ & $\square$ & $\square$ & $\square$ & $\square$ & $\square$ & $\square$ \\
\hline Prüf- und Materialkonzept für $\S 77 i$ Abs. 7 TKG & $\square$ & $\square$ & $\square$ & $\square$ & $\square$ & $\square$ \\
\hline Broschüre Verlegetechniken & $\square$ & $\square$ & $\square$ & $\square$ & $\square$ & $\square$ \\
\hline Abwasser: Hinweispapier des VKU und Merkblatt der DWA & $\square$ & $\square$ & $\square$ & $\square$ & $\square$ & $\square$ \\
\hline
\end{tabular}


C.8 Für wie hilfreich schätzen Sie die Zusammenarbeit mit den folgenden Partnern für Unterstützung, Planung oder Umsetzung eines Breitbandausbauprojektes ein?

\begin{tabular}{|l|c|c|c|c|c|}
\cline { 2 - 5 } \multicolumn{1}{l|}{} & $\begin{array}{c}\text { nicht hilf- } \\
\text { reich }\end{array}$ & $\begin{array}{c}\text { eher nicht } \\
\text { hilfreich }\end{array}$ & $\begin{array}{c}\text { teils / } \\
\text { teils }\end{array}$ & $\begin{array}{c}\text { eher hilf- } \\
\text { reich }\end{array}$ & $\begin{array}{c}\text { sehr hilf- } \\
\text { reich }\end{array}$ \\
\hline staatliche oder öffentlich geförderte Beratungsangebote & $\square$ & $\square$ & $\square$ & $\square$ & $\square$ \\
\hline privates Beratungsunternehmen oder Planungsbüro & $\square$ & $\square$ & $\square$ & $\square$ & $\square$ \\
\hline Telekommunikationsunternehmen / Netzanbieter & $\square$ & $\square$ & $\square$ & $\square$ & $\square$ \\
\hline Tiefbauunternehmen & $\square$ & $\square$ & $\square$ & $\square$ & $\square$ \\
\hline andere Kommunen & $\square$ & $\square$ & $\square$ & $\square$ & $\square$ \\
\hline Bürger & $\square$ & $\square$ & $\square$ & $\square$ & $\square$ \\
\hline Sonstige: & $\square$ & $\square$ & $\square$ & $\square$ & $\square$ \\
\hline
\end{tabular}

\begin{tabular}{|c|}
\hline $\begin{array}{c}\text { weiß } \\
\text { nicht }\end{array}$ \\
\hline$\square$ \\
\hline$\square$ \\
\hline$\square$ \\
\hline$\square$ \\
\hline$\square$ \\
\hline$\square$ \\
\hline
\end{tabular}


Wir bedanken uns für Ihre Teilnahme! Anhand der gewonnen Daten wird das KOMKIS im Anschluss der Befragung eine Analyse zum Breitbandausbau in Sachsen erstellen. Die Publikation wird Innen kostenfrei auf unserem Internetauftritt zum Herunterladen zur Verfügung stehen.

SACHSEN 
Das Kompetenzzentrum für kommunale Infrastruktur Sachsen (KOMKIS) stellt eine kostenfreie Informations- und Beratungsplattform für die kommunale Ebene in Sachsen dar. Im Schnittstellenbereich zwischen Verwaltung, Wissenschaft und Politik agiert das KOMKIS als kompetenter Informationsgeber, neutraler Vermittler und inhaltlicher Ansprechpartner zu Themen der kommunalen Infrastrukturbeschaffung, -erhaltung und -bewirtschaftung.

Weitere Veröffentlichungsformate abrufbar unter www.uni-leipzig.de/komkis:

\section{KOMKIS Dialog}

\section{KOMKIS Position}

\section{KOMKIS Praxis}

\section{KOMKIS Report}

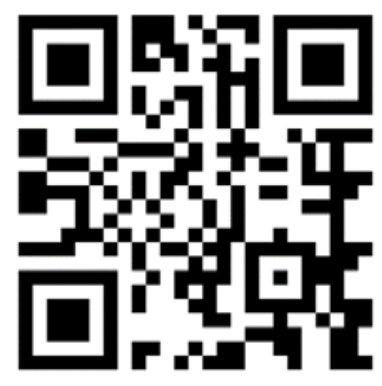

